\title{
$S B$
}

945

Cs 


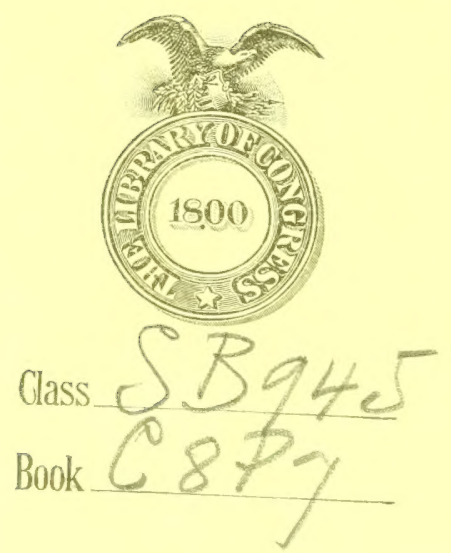








\title{
U. S. DEPARTMENT OF AGRICULTURE,
} BUREAU OF ENTOMOLOGY-BULLETIN NO, 100.

L. O. HOWARD, Entomologist and Chief of Bureau.

\section{THE INSECT ENEMIES OF THE COTTON BOLL WEEVIL.}

\author{
BY \\ W. DWIGHT PIERCE, \\ Agent and Expert, \\ ASSISTED BY \\ R. A. CUSHMAN AND C. E. HOOD, \\ Agents and Experts, \\ UNDER THE DIRECTION OF \\ W. D. HUNTER, \\ Agent and Expert, \\ In Charge of Southern Field Crop Insect Investigations.
}

ISSUED APRIL 3, 1912.

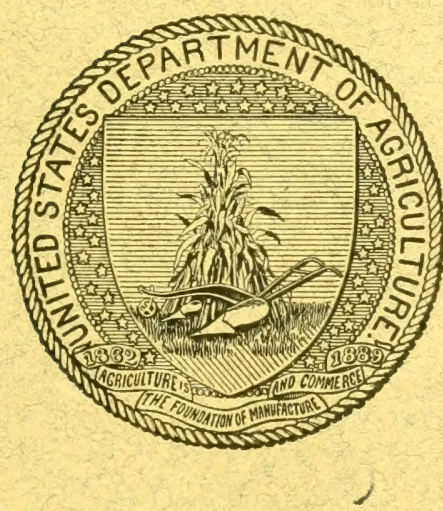

WASHINGTON:

GOVERNMENT PRINTING OFFIOE. 1912. 


\section{U. S. DEPARTMENT OF AGRICULTURE,}

BUREAU OF ENTOMOLOGY-BULLETIN No. 100.

L. O. HOWARD, Entomologist and Chief of Bureau.

\section{THE INSECT ENEMIES OF THE COTTON BOLL WEEVIL.}

BY

\section{W. DWIGH'T PIERCE,} Agent and Expert,

ASSISTED BY

R. A. CUSHMAN and C. E. HOOD, Agents and Experts,

UNDER THE DIRECTION OF

W. D. HUNTER, Agent and Expert,

In Charge of Southern Field Crop Insect Investigations.

ISSUED APRIL 3, 1912.

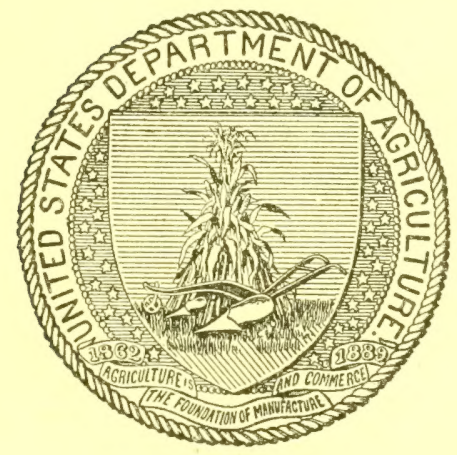

WASHINGTON:

GOVERNMENT PRINTING OFFICE. 1912. 


\section{$S B 945$ C8P7}

\section{BUREAU OF ENTOMOLOGY.}

L. O. Howard, Entomologist and Chief of Bureau.

C. L. Martatt, Entomologist and Acting Chief in Absence of Chief.

R. S. Curfton, Executive Assistant.

W. F. TAstet, Chief Clerk.

F. H. Chittenden, in charge of truck crop and stored product insect investigations.

A. D. Hopkins, in charge of forest insect investigations.

W. D. Hunter, in charge of southern field crop insect investigations.

F. M. WEBSTER, in charge of cereal and forage insect investigations.

A. L. QUAINTANCE, in charge of deciduous fruit insect investigations.

E. F. Philuips, in charge of bee culture.

D. M. RogERs, in charge of preventing spread of moths, field work.

Rolua P. Currie, in charge of editorial work.

Mabel ColCord, in charge of library.

\section{Southern Field Crop Insect Investigations.}

W. D. Hunter, in charge.

W. D. Pierce, J. D. Mitchell, G. D. Smith, E. A. Mcgregor, Harry Pinkus, W. A. Thomas, D. C. Parman, B. R. Coad, engaged in cotton boll weevil investigations.

F. C. Bishopp, A. H. Jennings, H. P. Wood, W. V. King, G. N. Wolcott, engaged in tick life-history investigations.

A. C. Morgan, G. A. Runner, S. E. Crumb, engaged in tobacco insect investigations.

J. L. WebB, T. E. Holloway, E. R. BARber, engaged in sugar cane and rice insect investigations.

R. A. Cooley, D. L. Van Dine, Wilmon Neweli, A. F. Conradi, C. C. Krumbiaar, collaborators.

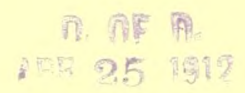




\title{
LETTER OF TRANSMITTAL.
}

\author{
U. S. Department of Agriculture, \\ Bureat of Entomology, \\ Washington, D. C., September 28, 1911.
}

Sin: I have the honor to transmit herewith a manuseript entitled "The Insect Enemies of the ('otton Boll Weevil," by Messrs. W. Dwight Pierce, R. A. ('ushman, and ('. E. Hood, agents and experts engaged in cotton boll weevil investigations. The present manuscript contains a complete summary of the studies of the boll-weevil parasites conducted since 1905. The boll weevil is now known to be attacked by 29 species of parasites and 26 species of predatory insects, most of which are indigenous to the United States. It is the purpose of this manuseript to show the sourees and value of these enemies and to indicate how they may be utilized to the advantage of the farmers of the cotton belt. I recommend the publication of this manuscript as Bulletin No. 100 of the Bureau of Entomology.

Respectfully,

L. O. HowaRd, Entomologist and Chief of Bureau.

Hon. James Wilson, Secretary of Agriculture. 



\section{CONTENTS.}

Page.

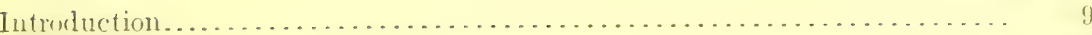

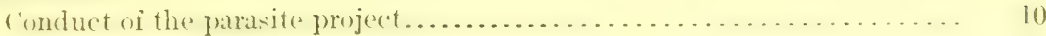

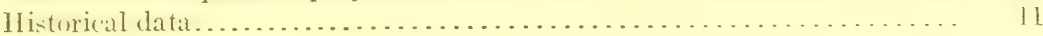

Serpe of present report . . . . . . . . . . .

Part I. The status of the cotton boll weevil and its enemies.

1. A general chronological study of the insect control of the boll weevil.. I: li

2. Nature and sources of the material examined ................. It

3. Seasonal studies of insect control, by class of infested material...... I It

4. A geographic study of the statistics of insect control . . . . . . . . . . . 2 2)

5. A study of the share of insect control in the mortality of immature boll

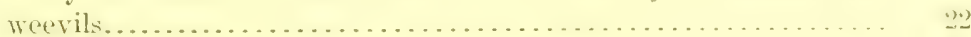

6. A study of how agriculture modifies insect control .............. . 30

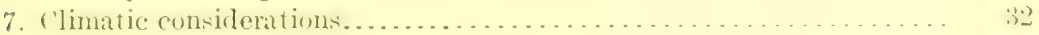

S. How insect control follows the dispersion of the boll weevil . . . . . . . . . : .

9. The status of the boll weevil and its control by insects . . . . . . . . . :37

10. A brief statement of the various classes of control exercised upon the

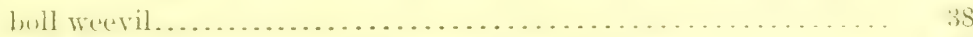

11. Practical conclusions derived from statistical studies............ 3 s

Part II. Biological complex.

1. A list of the insect anemies of the cotton boll weevil............. . 40

2. The hosts of boll-weevil parasites.............

3. Mites which attack the boll weevil....................... 43

4. Flies which parasitize the boll weevil ..................... . . 47

5. The hymenopterous parasites of the boll weevil............... . . 48

6. Biological notes upon the parasites of the weevil ............... it

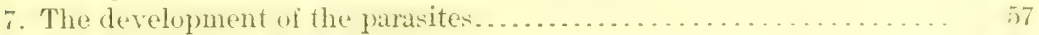

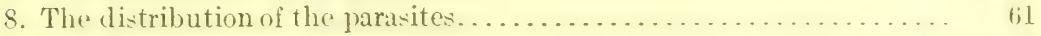

9. The parasite seasons. . . . . . . . . . . .

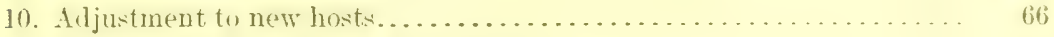

11. Beetles which prey upon the boll weevil...................

12. Lepidopterous larva which are incidentally predatory upon the boll weevil...................................

13. Ants which prey upon the boll weevil........................ . . .

14. Biology of the cohosts of the boll-weevil parasites.................

15. A list of the host plants of the cohost weevils................. si)

16. A summary of the more important biological facts ..............

Part III. The economic application.

1. The economic principles involved. . . . . . . . . . . . . . . . .

2. Interpretation of parasite statistics........................

3. Interpretation of the biological complex . . . . . . . . . . . . . . . . . 8 i

4. How to profit by existing conditions. . . . . . . . . . . . . . . . . . .

5. How to plan for the greatest possible control...................

6. Propagation and artificial introductions.................... yl

7. Objectionable practices............................... 93

8. The economic significance of the investigation ............... 94

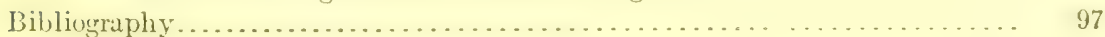




\section{L L US TRATIONS.}

PLATES.

Plate I. Shedding and retention of forms on the cotton plant. Fig. 1.-Normal scars left by shed forms. Fig. 2.-Abnormal scars with forms retained. Fig. 3,-Longitudinal section through base of retained form . . . . . . . . . . . . . . . . . . . . . . . . . . .

II. Eggs of boll-weevil parasites. Fig. 1,-1ficrodontomerus anthonomi. Fig. 2,-Unidentified egg. Fig. 3.-Cerambycobius cyaniceps. Fig. 4.-Eurytoma tylodermatis. Fig, 5.-Catolaccus hunteri. Fig. 6.-Unidentified egir . . . . . . . . . . . . . . . . . . . . . .

III. Parasites of weevils. Fig. 1.-Eurytoma tylodermatis, pupa. Fig. 2.Catolaccus incertus, pupa. Fig. 3.-Cerambycobius cyaniceps, pupa. Fig. 4.-Microdontomerus anthonomi, pupa. Fig. 5.-Larva of Microbracon. Fig. 6.-Microbracon mellitor, pupa. Fig. 7.-Larva of chalcidoid

TEXT FIGURES.

FIG. 1. Diagram illustrating the monthly percentage of mortality of immature boll weevils due to insect enemies. .......................

2. Diagram illustrating the average climatic and insect control of the immature boll weevils during $1906,1907,1908$, and 1909 , in hanging

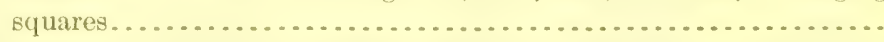

3. Diagram illustrating the average climatic and insect control of the immature boll weevils during $1906,1907,1908$, and 1909, in fallen squares.

4. Diagram illustrating Texas climatic variations in 1907, 1908, and 1909 .

5. Diagram illustrating Louisiana climatic variations in 1907, 1908, and 1909 .

6. Diagram illustrating the boll-weevil complex ..................

7. Diagram giving the parasites of the boll weevil and their other hosts.

8. Pediculoides ventricosus: Adult female, beiore and after inflation of abdomen with eggs and young . . . . . . . . . . . . . . . . . . . .

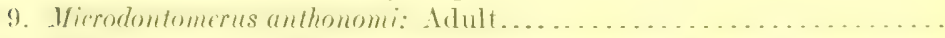

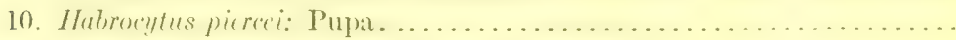

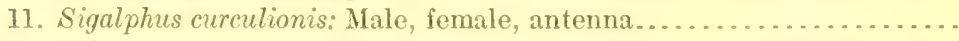

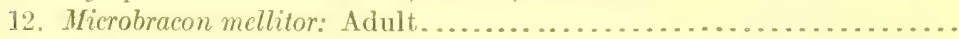

13. Diagram illustrating yearly rank of the boll-weevil parasites, 1906 , 1907,1908 , and 1909.

14. Map showing the distribution of the more important parasites of the boll weevil

15. Diagram illustrating the seasonal rotation of hosts of Catolaccus hunteri

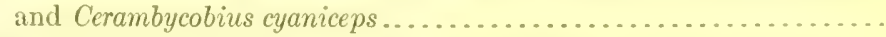

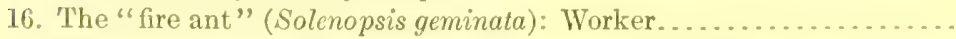

17. The little black ant (Monomorium minimum): Adult, egg, larva, pupa . 
Fig. 18. The little red ant (Monomorium pharaonis): Female and worker.... T.

19. The coffee-bean weevil (Arxcerus fasciculatus): Adult, larva, pupa... it

20. The bloodweed weevil (Lixus serobicollis): Adult................ 75

21. The ironweed weevil (Desmoris scapalis): Adult................. 76

22. The pepper weevil (Anthonomus eugenii): Adult............... i7

23. The cowpea weevil (Chalcodermus æncus): Adult................ is

24. The plum curculio (Conotrachelus nenuphar): Larva, adult, pupa.... 78

25. The potato-stalk weevil (Trichobaris trinotata): Adult, larvæ, pupæ.. $\quad$ i9

26. The rice weevil (Calandra oryza): Adult..................... 80 



\section{THE INSECT ENEMIES OF THE COTTON BOLL WEEVIL.}

\section{INTRODUCTION.}

When the cotton boll weevil first entered the United States it appeared to have almost undisputed sway. It did, in fact, escape most of its enemies. But whether parasites were introdued with it or not, we now know that within the first thee years of its exist ence in Texas it was attacked by three important species of parasites. Year after year new factors in its control are becoming apparent, although some have probably been concerned since the beginning. () n the ot her hand, it is certain that entirely new elements are cut cring the struggle as rapidly as the weevil enter's new biological complexes. Among the most striking of these new elements in the control is the recent aul justment of Microdontomoms anthonomi (rawford (fig. 9, p. 49). This spe(ies was unknown until 1906 when a very few were taken in material reared at Cuero, Goliad, Irallettsville, Vietoria, and Waco, Tex. In 1907 it was found to predominate in a portion of central Texas. In 1908 its range was found to extend northward to the Red River. In 1909 it was found as far east as the Mississippi River in Louiciana. Only a single record of the occurrence of Sigalplus curcutionis Fitch (fig. 11, p. 53), the common parasite of the plum (ureulis, had been made prior to 1908 . In that year it began to make its presence felt in northeastem Louisiana and western Mississippi. In 1908 a new chalcidoid parasite, recently described as Tetrastichus hunteri Craw ford, was found to be the leading parasite of the boll weevil in northern Louisiana and western Mississippi. In 1909 this species wats found as far west as Arlington, Tex.

With the advent of each new enemy and its more complete adjustment, the power for damage possessed by the weevil is by so much diminished. On the other hand, every factor which checks these enemies without also checking the weeril benefits the weeril.

If the solution of the boll-weeril problem consistel merely in adeling, one by one, factors which would cut off a given percentage of the weevils, the time would not be distant when that might be aceomplished. The problem is far more complicated. The various factors do not discriminate agrainst one another, for while hear, ants, cold weather, and excessive moisture may remove many parasites from the struggle, it also follows that heat, cold weather, heary rains, 
predators, and other pests remove many ants. Proliferated plant tissue frequently acts as an important check upon the weevil, and yet in many cases it serves as a ford for the weevil rather than as a controlling forec. Mroist weather aids the weevil. The leaf-worm, while cutting of most of the weevil food, finally is checked by parasites. light, heat, and dryness favor certain parasites, whereas shade and moisture favor others. Parasites that are normally primary frequently waste their energies by accidentally becoming secondary, and normally secondary parasites, and probably tertiary as well, also enter the consideration. Predatory enemies fail to distinguish the parawites from the weerils, but are in turn held in check by their own enemies. The birds devour weevils, predators, and parasites, but they in turn are kept down by other birds and even man himself, and thus the complexity grows.

There are 49 species of inserts which attack the immature stages of the boll weevil. (Of these insects 2!) species may be classed as parasitic, 5 as predatory larvæ, and 15 as predatory adults. They are divided among the orders, with 3 in the Acarina, 4 in Coleoptera, 36 in Hymenoptera, 5 in Diptera, and 1 in Lepidoptera. One of the acarians, 1 coleopteron, 15 Hymenoptera, and 1 dipteron, may be considered as quite important. A weighted average mortality of 3.93 per cent of all immature stages may be aecredited to the combined faretors of all parasitic enemies; the predators are responsible for 15.9.3 pere cent, while climate is responsible for 24.45 per cent, and plant proliferation 12.42 per' cent. This makes the total natural control of immature stages 56.73 per cent.

In addition to the insects attacking the boll weevils in the squares, there must be considered the insects which prey on the adults. These include one praying mantis, one predaceous bug, two beetles, and two ants-six species in all.

This bulletin is not concerned with the mortality of the weevils after they luecone adult becalse reliable figures can not be gathered upon this point. It is certain that many weevils fall prey to predaceous insects and to birds, and the well-known habits of the horned lizard would include it in the list of possible enemies. The important fiact is that 56 pere cent of the weeril eggess fail to produce adult weevils. The remainder is still a formidable number but the many adverse influences continue to operate upon the adults and likewise upon their progeny.

\section{CONDUCT OF THE PARASITE PROJECT.}

The investigation of the insect enemies of the cotton boll weevil was initiated in 1905. It has been conducted from the beginning by the senior author under the direction of Mr. W. D. Hunter, and with 
the direct influence and encouragement of Dr. L. O. Howard, (hief of the Bureau of Entomology. Messrs. R. A. Cushman and C. E. Inood have been intimately associated in the preparation of the material for this bulletin since 1907. The collecting, examining, and recording of the immense mass of material has involved in addition the services of Messrs, E. A. Schwarz, J. D. Mitchell, W. E. Hinds, Wilmon Newell, F. C. Bishopp, A. C. Morgan, F. C. Pratt, ('. R. Jones, W. W. Yothers, G. D. Smith, A. H. Rosenfeld, II. S. Smith, E. S. Tucker, T.C.Barber, S. Goes, C. S. Spooner, C. W. Flymn, T. C. Paulsen, J. A. Hyslop, V. I. Safro, T. E. Holloway, H. Pinkus, W. H. Hoffman, F. L. Elliott, and O. M. Lander. Considerable eredit is due Messis. E. A. Schwarz, J. C. Crawford, W. M. Wheeler, D. W. Coquillett, and C. H. T. Townsend for determination of the insects concerned. The weevils entering the biological complex have been determined by the senior author. In short, 33 entomologists have directly contributed the data which are herewith presented.

HISTORICAI, DATA.

The first definite records of the parasites of the cotton boll weevil were made by C. II. T. Townsend in 1895 when he mentioned a small hymenopterous parasite and also recorded the suspicious occurrence of sereral species of S'ymmus in the squares, and mentioned that a fungoid parasite, a species of (ordyceps, "was found growing out of a dead pupa in its cell in a boll, November 26, in a field in san Juan Allende, Mexico." (Townsend, 1895.) In 1901 Profs. Herrera and Rangel published notes concerning the parasitic attack of Ptdiculoides ventricosus Newport (fig. 8, p. 46) upon the boll weevil (Rangel, 1901b, 1901c).

In 1902 Dr. Wm. II. Ashmead deseribed Bruchophugus herrere from ('oahuila, Mex., as a primary parasite of the boll weevil (Ashmead, 1902a, 1902b; THerrera, 1902a). This is probably- Eurytoma tylod rmatis Ashmead. Prof. Herrera also recorded the activities of a predacens ant, Formica fusca Linn., subspecies subpolita Mayrr, variety perpilosa Whecler, (Herrera, 1902b; Wheeler, 1902). In the same year Prof. F. W. Mally reconded the fact that Bracon (now Microbracon) mellitor Say (fig. 12, p. 5t) and Eupelmus (now ('erambycobius. cyaniceps A. meat had been reared by him, since 1899, in considerable numbers from the boll weevil. He also recorded a species of Eurytoma. (Mally, 1902.)

In $190+$ IIunter and Hinds recorded additional primary parasites as follows: Sigalphus curculionis Fitch (fig. 11, p. 53), Catolaceus incertus Ashmead, Trosigalphus (robustus Ashmead), Bracon (dorsator Say), and Eurytoma tylodermatis Ashmead, as well as an entomogenous fungus, Aspergillus (Iunter and Ilinds, 1904). The Lrosi- 
galphus has since heen described as Urosigal pluns anthonomi Craword (Crawford, 1907a).

In 1906 Mr. Nathan Banks deseribed a mite, Tyrortyphus breviceps, collected at Victoria, Tex., from boll-weevil larva (Banks, 1906).

In 1907 the senior author of this bulletin added Hydnocera pubescens Leconte als a predaceous enemy of the boll weevil (Pierce, 1907a, 1907b, $1907 \mathrm{c})$. In the same year Dr. Hinds published two papers in which the work of Solenopsis geminata Fab. (fig. 16, p. 70), variety xyloni Mrchok, as a predator on the boll weevil was fully discussed, and (onsiderable statistical data on the parasitic control of the weevil were presented (Hinds, 1907a, 1907b). Mr. Morgan published a brief account of the predatory attack of a bug, Apiomerus spissipes Say (Morgan, 1907). Mrr. J. C. Crawford described as parasites of the holl weevil Torymus anthonomi, Crosigalphus anthonomi, and Urosigalphus schwarzi (Crawford, 1907a).

In 1908 the senior wuthor of this report recorded Catolaccus anthonomi Awhmead as a boll-weevil parasite and Cathartus cassix Reiche (gemetlutus Dural) als al predator" (Pierce, 190Sa, 1908b, 1908c, 1908d). Mr. Crawford described Cerambycobius cushmani and C'atolaccus Tunteri as new parasites of the boll weevil (Crawford, 1908). During the same year two new predaceous enemies of the boll weevil were recorded from Louisiana, namely, Evarthrus sodalis LeConte and Evarthrus sp. (Newell and Trehearne, 1908). Mr. Townsend, in a paper on the muscoidean flies, recorded Ennyomma ylobosa Townsend as a parasite of the boll weevil (Townsend, 1908). During $1909 \mathrm{Mr}$. (rawford desscribed Tetrastichus hunteri as a parasite of the boll weevil (Crawford, 1909b).

SCOPE OF PRESENT REPORT.

The present report is supplementary to a former bulletin which was based on investigations prior to 1907 (Pierce, 1908a). The matter contained herein has mainly been gathered during the years $190 \%, 1901$, and 1909. Only such notes as are of value for the sake of comparison have been repeated from the previous report.

The work is divided into three parts:

I. The status of the cotton boll weevil and its enemies.

II. The biological complex.

III. The economic application.

\section{PART X. THE STATUS OF THE COTTON BOLI WEEVIL AND ITS ENEMIES.}

Part I of this bulletin shows the large mass of statistical material gathered during the four years of the parasite investigation, and attempts to place this material in such form as to show its economic value and significance. 
The matter is arranged topically as follows:

1. A general chronological study of the insect control of the boll weevil.

2. The nature and sources of the material examined.

3. Seasonal studies of the insect cont rol by class of infest ed mat rerial.

4. A geographical study of the statistics.

5. A study of the share of insect control in the mortality of immature weevils.

6. A study of how agriculture modifies insect control.

7. Climatic considerations.

8. How insect control follows the weevil dispersion.

9. The status of the boll weevil and its control by insects.

10. A brief statement of the various classes of control exereised upon the weevil.

11. Practical conclusions derived from statistical studies.

1. A General CHronological STUDY OF THE INSECT CONTROL OF THE BOLL IVEEVIL.

Records upon parasitic control of the boll weevil begin in July, 1902, and occur more or less scatteringly until June, 1906. The records of 1906 were very extensive, but, as will be shown, they were merely preliminary.

Table $\mathrm{I}$ is arranged to show the extent of the exminations male since 1902. This table should not be used to compare the records of the various years, as the manner of investigation in each year has been different, and the sources of the material have raried greatly. The table is only intended to show the tot al of the examinations and how these were distributed from year to year. A careful analysis of the figures has been given in the various seetions of Part I.

TABLE I.--Insect control of the boll weevil, by years.

\begin{tabular}{|c|c|c|c|c|c|c|}
\hline \multirow{2}{*}{ YLAR. } & \multirow{2}{*}{$\begin{array}{l}\text { WVeril } \\
\text { stages. }\end{array}$} & \multirow{2}{*}{$\begin{array}{l}\text { Preda- } \\
\text { tors. }\end{array}$} & \multirow{2}{*}{$\begin{array}{l}\text { Para- } \\
\text { sites. }\end{array}$} & \multicolumn{3}{|c|}{ 'Per cent mortality due to- } \\
\hline & & & & $\begin{array}{c}\text { Preda- } \\
\text { tors. }\end{array}$ & $\begin{array}{l}\text { Para- } \\
\text { sites. }\end{array}$ & $\begin{array}{c}\text { All } \\
\text { inserts. }\end{array}$ \\
\hline 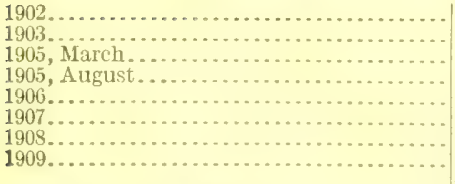 & $\begin{array}{r}602 \\
819 \\
1,005 \\
1,702 \\
40,073 \\
13,602 \\
29,349 \\
11,653\end{array}$ & $\begin{array}{l}(?) \\
(?) \\
(?) \\
(?) \\
10,547 \\
2,279 \\
3,862 \\
1,231\end{array}$ & $\begin{array}{r}7 \\
59 \\
32 \\
21 \\
1,728 \\
1,121 \\
2,952 \\
620\end{array}$ & $\begin{array}{l}(?) \\
(?) \\
(?) \\
(?) \\
26.31 \\
16.75 \\
13.15 \\
10.56\end{array}$ & $\begin{array}{r}1.16 \\
7.20 \\
3.18 \\
1.23 \\
4.31 \\
8.24 \\
10.05 \\
5.32\end{array}$ & $\begin{array}{l}(?) \\
(?) \\
(?) \\
(?) \\
30.62 \\
24.99 \\
23.20 \\
15.88\end{array}$ \\
\hline Totals and averages............... & 98,805 & $\ldots \ldots . .$. & 6,540 & .... & 16.61 & $\ldots$ \\
\hline
\end{tabular}

${ }^{1}$ This table should not be construed as indicating that parasite control has been falling off, because the table is based upon different kinds of examinations in different years. The detailed analysis of these records will be found on subsequent pages. 
The figures of 1902 are based on the total number of stages reared. The data of 1903 are based on the total number of stages reared, but include both stages in hanging and lallen forms. There were 654 stages in fallen forms of which 14 , or 2.14 per cent, were parasitized, and 165 stages in hanging forms of which 45 , or 27.27 per cent, were parasitized. The figures of 1905 were separated to show the investigations of March and August because of the great difference in the mortality from parasites in these two months. Between 1906 and 1909 the data represent all classes of infested material and all infested regions. It will be noticed that for the last four years the total insect control of the immature weevils has fluctuated between 15 and 30 per cent.

\section{NATURE AND SOURCES OF THE MATERIAL EXAMINED.}

During the four years 1906-1909 examinations of mortality have been made of material collected at 6 places in Arkansas, 26 in Louisiana, 6 in Mississippi, 7 in Oklahomal, and 65 in Texas, making a total of 110 places. These examinations are based upon 94,677 stages, involving an individual examination of over 222,700 cotton forms (squares, blooms, and bolls). Many other collections and examinations were made, but because of incomplete records are excluded from the accompanying tables.

During the four years there has been the equivalent of examinations in 176 localities, or an average of 44 localities per year.

\section{SEASONAL STUDIES OF INSECT CONTROL, BY CLASS OF INFESTED MATERIAL.}

Very shortly after the work began in 1906 it became evident that the actirity of the parasites and other insect enemies of the weevil was very different in squares and bolls, and in fallen or hanging squares ir bolls, and also that the highest control by parasites was in hanging squares.

An examination of the squares of various varieties of cotton plants will show the ohserver that certain ones have a transverse at tachment of the pedicel to the stem. In all cases where this attachment is perfectly transverse, the square when injured by any insect is caused to drop because of the separation of the infested part from the main stalk hy the growth of an absciss layer at the point of attachment. (Pl. I, fig. 1; fig. 3, a.) Certain other varieties indicate a long diagonal attachment to the stem. When these squares are injured, a diagonal absciss layer is formed which runs down the stem from onehalf to three-fourths of an inch or even more. This layer is gencrally incomplete at the lower point and consequently the square 
hangs by a few threads and dries on the plant. (Pl. I, fig. 2; fig. 3, b.) To these squares and bolls which thus hangs, we have applied the terms "hanging squares" and "hanging bolls."

Tables II and III, which are arranged to show the monthly percentages of control by parasites, by predators, and by all insects, illustrate the differences in the control of the weevil in the four principal classes of infested material, namely, lallen and hanging dry squares, and fallen and hanging dry bolls.

$\Lambda$ few words of explanation of these tables are necessary in order to show what is meant by the different classes of mortality. It has been found that a large number of stages are destroyerl as egors or young larva by the prolileration of the plant tisicues. It the time of the examination for mortality of the weevil, all evidence of the weerils destroyed in these stages has disappeared; consequently the percentages of mortality given in the following tables are the percentages of stages found which are killed br the aunses cnumerated, and the mortality from proliferation is entirely ignored.

TABLE II.-Monthly mortality of the boll weeril due to inscets in fallen squares.

\begin{tabular}{|c|c|c|c|c|c|c|c|c|c|}
\hline \multirow{2}{*}{ Month. } & \multirow{2}{*}{$\begin{array}{l}\text { Squares } \\
\text { exam- } \\
\text { ined. }\end{array}$} & \multirow{2}{*}{$\begin{array}{l}\text { Stages. } \\
\text { found. }\end{array}$} & \multicolumn{3}{|c|}{ Stages killed hy- } & \multicolumn{4}{|c|}{ Pereentage of stages killed. } \\
\hline & & & $\begin{array}{c}\text { Cli- } \\
\text { mate. }\end{array}$ & $\begin{array}{l}\text { Preda- } \\
\text { tors. }\end{array}$ & $\begin{array}{l}\text { Para- } \\
\text { sites. }\end{array}$ & Total. & $\begin{array}{c}\text { Preda- } \\
\text { tors. }\end{array}$ & $\begin{array}{l}\text { Para- } \\
\text { sites. }\end{array}$ & $\begin{array}{c}\text { All } \\
\text { insects. }\end{array}$ \\
\hline 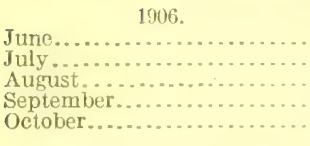 & $\begin{array}{r}4,476 \\
7,205 \\
19,301 \\
10,709 \\
1,991\end{array}$ & $\begin{array}{r}3,831 \\
4,552 \\
11,186 \\
5,365 \\
6009\end{array}$ & $\begin{array}{l}1,797 \\
1,676 \\
2, \times 25 \\
1,475 \\
205\end{array}$ & $\begin{array}{r}914 \\
1,079 \\
4,097 \\
1,625 \\
133\end{array}$ & $\begin{array}{r}115 \\
207 \\
180 \\
285 \\
33\end{array}$ & $\begin{array}{l}73.8 \\
65.1 \\
63.5 \\
63.0 \\
61.8\end{array}$ & $\begin{array}{l}23.5 \\
23.6 \\
36.6 \\
30.2 \\
22.1\end{array}$ & $\begin{array}{l}3.1 \\
4.7 \\
\text { 1. } 7 \\
5.4 \\
5.6\end{array}$ & $\begin{array}{l}26.9 \\
28.3 \\
38.3 \\
35.6 \\
27.7\end{array}$ \\
\hline $\begin{array}{r}\text { Totais and averages for } \\
1906 \text {................. }\end{array}$ & 43,736 & 25,534 & $7,981 !$ & 7,548 & 823 & 6.5. 2 & 30.7 & 3.3 & 34.0 \\
\hline 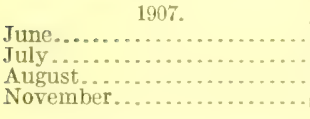 & $\begin{array}{l}2,074 \\
5,192 \\
7,400 \\
150\end{array}$ & $\begin{array}{r}1,261 \\
3,608 \\
5,058 \\
93\end{array}$ & $\begin{array}{r}339 \\
775 \\
2,156 \\
90\end{array}$ & $\begin{array}{r}198 \\
433 \\
1,372 \\
0\end{array}$ & $\begin{array}{r}76 \\
133 \\
155 \\
1\end{array}$ & $\begin{array}{l}48.6 \\
37.2 \\
72.8 \\
97.7\end{array}$ & $\begin{array}{r}15.6 \\
11.9 \\
27.1 \\
0\end{array}$ & $\begin{array}{l}7.0 \\
3.8 \\
3.0 \\
1.0\end{array}$ & $\begin{array}{r}22.6 \\
15.7 \\
30.1 \\
1.0\end{array}$ \\
\hline $\begin{array}{r}\text { Totals and averages for } \\
1907 \ldots \ldots \ldots \ldots \ldots\end{array}$ & $14, \$ 16$ & 10,020 & 3,363 & 2,003 & 365 & 57.1 & 19. 9 & 3.7 & 23.6 \\
\hline 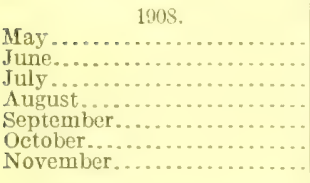 & $\begin{array}{r}100 \\
7,808 \\
7,437 \\
1,911 \\
7,189 \\
9,678 \\
604\end{array}$ & $\begin{array}{r}56 \\
5,285 \\
4,690 \\
1,208 \\
3,894 \\
5,342 \\
369\end{array}$ & $\begin{array}{r}4 \\
1,169 \\
1,047 \\
540 \\
578 \\
807 \\
90\end{array}$ & $\begin{array}{r}0 \\
866 \\
902 \\
294 \\
815 \\
259 \\
1\end{array}$ & $\begin{array}{r}2 \\
324 \\
219 \\
6.3 \\
136 \\
659 \\
88\end{array}$ & \begin{tabular}{l|l}
10.7 \\
44.6 \\
46.2 \\
74.2 \\
39.2 \\
32.8 \\
51.1
\end{tabular} & $\begin{array}{r}0 \\
16.4 \\
19.2 \\
24.3 \\
20.9 \\
5.4 \\
2.7\end{array}$ & $\begin{array}{r}3.5 \\
6.1 \\
4.7 \\
5.2 \\
3.5 \\
12.3 \\
22.6\end{array}$ & $\begin{array}{r}3.5 \\
22.5 \\
23.9 \\
29.5 \\
24.4 \\
17.7 \\
25.3\end{array}$ \\
\hline $\begin{array}{r}\text { Totals and a veraces for } \\
1908 \ldots \ldots\end{array}$ & 34,757 & 20,844 & 4,235 & 3,167 & 1,491 & 42. 7 & 15. 2 & 7.1 & 22.3 \\
\hline 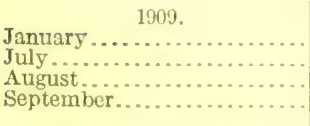 & $\begin{array}{r}50 \\
7,677 \\
5,507 \\
750 \\
\end{array}$ & $\begin{array}{r}23 \\
4,334 \\
2,866 \\
364\end{array}$ & $\begin{array}{r}0 \\
1,318 \\
680 \\
19\end{array}$ & $\begin{array}{r}0 \\
507 \\
425 \\
5\end{array}$ & $\begin{array}{r}0 \\
131 \\
39 \\
1\end{array}$ & $\begin{array}{r}0 \\
45.1 \\
39.9 \\
\text { 6. } 8\end{array}$ & $\begin{array}{r}0 \\
11.7 \\
14.9 \\
1.4\end{array}$ & $\begin{array}{r}0 \\
3.0 \\
1.3 \\
.3\end{array}$ & $\begin{array}{r}0 \\
14.7 \\
15.2 \\
1.7 \\
\end{array}$ \\
\hline $\begin{array}{l}\text { Totals and averages for } \\
1909 \ldots \ldots \ldots . . . . . . . . . .\end{array}$ & 13,984 & 7,587 & 2,017 & 940 & 170 & 41.2 & 12.4 & 2.2 & 14.6 \\
\hline
\end{tabular}


A study of Table II, on the mortality due to insects in fallen squares, shows that the principal insect work is that of predatory insects and, furthermore, that the total insect control is, as a rule, less than the climatic control. The table embraces the examination of $6: 3,985$ weeril stages of which 17,596 were killed by climate, 13,958 by predators, and 2,849 by parasites. In other words, the average pereentage of control in fallen squares by all kinds of insects is 26.2 per cent, or 21.8 per cent by predators and 4.4 per cent by parasites.

1 further study of Table II shows that the predators have in each year done their most valuable work in the month of August. The parasites, however, have shown considerable variation in the month of their best work. In 1906 the highest average percentage was in October; in 1907 it was in .June; in 1908 in November; and in 1909 in July.

TABLE III.-Monthly mortality of the boll weevil due to insects in hanging squares.

\begin{tabular}{|c|c|c|c|c|c|c|c|c|c|}
\hline \multirow[b]{2}{*}{ Months. } & \multirow{2}{*}{$\begin{array}{c}\text { Squares } \\
\text { exam- } \\
\text { ined. }\end{array}$} & \multirow{2}{*}{$\begin{array}{l}\text { Stages } \\
\text { found. }\end{array}$} & \multicolumn{3}{|c|}{ Stages killed by- } & \multicolumn{4}{|c|}{ Percentage of stages killed. } \\
\hline & & & $\begin{array}{l}\text { Cli- } \\
\text { mate. }\end{array}$ & $\begin{array}{l}\text { Preda- } \\
\text { tors. }\end{array}$ & $\begin{array}{l}\text { Para- } \\
\text { sites. }\end{array}$ & Total. & $\begin{array}{l}\text { Preda- } \\
\text { tors. }\end{array}$ & $\begin{array}{l}\text { Para- } \\
\text { sites. }\end{array}$ & $\begin{array}{l}\text { All in- } \\
\text { sects. }\end{array}$ \\
\hline 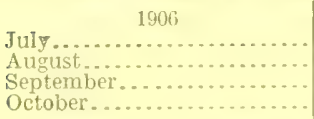 & $\begin{array}{r}474 \\
4,165 \\
2,032 \\
33\end{array}$ & $\begin{array}{r}247 \\
2,566 \\
1,285 \\
20\end{array}$ & $\begin{array}{r}47 \\
539 \\
245 \\
4\end{array}$ & $\begin{array}{r}20 \\
544 \\
269 \\
10\end{array}$ & $\begin{array}{r}76 \\
337 \\
125 \\
0\end{array}$ & $\begin{array}{l}57.9 \\
55.3 \\
49.7 \\
70.0\end{array}$ & $\begin{array}{r}8.0 \\
21.2 \\
20.9 \\
50.0\end{array}$ & $\begin{array}{r}21.0 \\
13.1 \\
9.7 \\
0\end{array}$ & $\begin{array}{l}29.0 \\
34.3 \\
30.6 \\
50.0\end{array}$ \\
\hline $\begin{array}{r}\text { Totals and averages for } \\
1906 . . . \ldots \ldots \ldots . . . . .\end{array}$ & 6,704 & 4,118 & 835 & 843 & 538 & 53.8 & 20.5 & 13.0 & 33.5 \\
\hline $\begin{array}{l}\text { June............. } \\
\text { July............. } \\
\text { August. . . . . . . . }\end{array}$ & $\begin{array}{r}150 \\
956 \\
3,5 \pm 3\end{array}$ & $\begin{array}{r}S 8 \\
513 \\
2,011\end{array}$ & $\begin{array}{r}26 \\
57 \\
296\end{array}$ & $\begin{array}{r}5 \\
16 \\
175\end{array}$ & $\begin{array}{r}13 \\
103 \\
580\end{array}$ & $\begin{array}{l}50.0 \\
34.3 \\
52.2\end{array}$ & $\begin{array}{l}5.7 \\
3.1 \\
8.7\end{array}$ & $\begin{array}{l}14.7 \\
20.1 \\
28.8\end{array}$ & $\begin{array}{l}20.4 \\
23.2 \\
37.5\end{array}$ \\
\hline $\begin{array}{r}\text { Totals and averages for } \\
1907 \ldots \ldots \ldots \ldots \ldots \ldots\end{array}$ & 4,649 & 2,612 & 379 & 196 & 696 & 48.6 & 7.5 & 26.6 & $\therefore 4.1$ \\
\hline 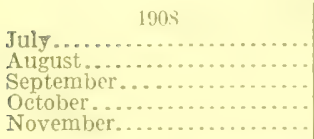 & $\begin{array}{l}2,955 \\
1,393 \\
3,922 \\
1,192 \\
1,125\end{array}$ & $\begin{array}{r}2,177 \\
842 \\
2,340 \\
553 \\
10\end{array}$ & $\begin{array}{r}395 \\
141 \\
476 \\
116 \\
8\end{array}$ & $\begin{array}{r}179 \\
91 \\
259 \\
53 \\
0\end{array}$ & $\begin{array}{r}479 \\
279 \\
410 \\
113 \\
0\end{array}$ & $\begin{array}{l}48.4 \\
60.7 \\
48.9 \\
52.9 \\
80.0\end{array}$ & $\begin{array}{r}8.2 \\
10.8 \\
11.0 \\
10.0 \\
0\end{array}$ & $\begin{array}{r}22.0 \\
33.1 \\
17.5 \\
21.2 \\
0\end{array}$ & $\begin{array}{r}30.2 \\
43.9 \\
28.5 \\
31.2 \\
0\end{array}$ \\
\hline $\begin{array}{l}\text { Totals and averages for } \\
1905 \text {...................... }\end{array}$ & 10,587 & 5,922 & 1,136 & 582 & 1,281 & 50.9 & 9.8 & 21.7 & 31.5 \\
\hline 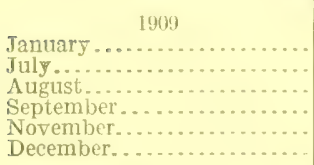 & $\begin{array}{r}80 \\
630 \\
1,290 \\
745 \\
136 \\
515\end{array}$ & $\begin{array}{r}3 \\
383 \\
856 \\
496 \\
52 \\
169\end{array}$ & $\begin{array}{r}0 \\
36 \\
96 \\
61 \\
14 \\
47\end{array}$ & $\begin{array}{r}0 \\
31 \\
72 \\
21 \\
0 \\
1\end{array}$ & $\begin{array}{r}0 \\
117 \\
88 \\
76 \\
30 \\
71\end{array}$ & $\begin{array}{r}0 \\
48.0 \\
29.9 \\
31.9 \\
100.0 \\
70.4\end{array}$ & $\begin{array}{r}0 \\
8.1 \\
8.4 \\
4.2 \\
0 \\
6\end{array}$ & $\begin{array}{r}0 \\
30.5 \\
10.3 \\
15.3 \\
68.0 \\
42.0\end{array}$ & $\begin{array}{r}0 \\
38.6 \\
18.7 \\
19.5 \\
68.0 \\
42.6\end{array}$ \\
\hline $\begin{array}{r}\text { Totals and averages for } \\
1909 \ldots \ldots \ldots \ldots \ldots \ldots\end{array}$ & 3,396 & 1,959 & 254 & 125 & 382 & 38.8 & 6.4 & 19.5 & 25.9 \\
\hline
\end{tabular}

Talble III shows that the principal insect work in hanging squares is that of parasitic insects and, furthermore, that the total insect control in hanging squares is each year higher than the climatic control. This table embraces the examination of 14,611 weevil 


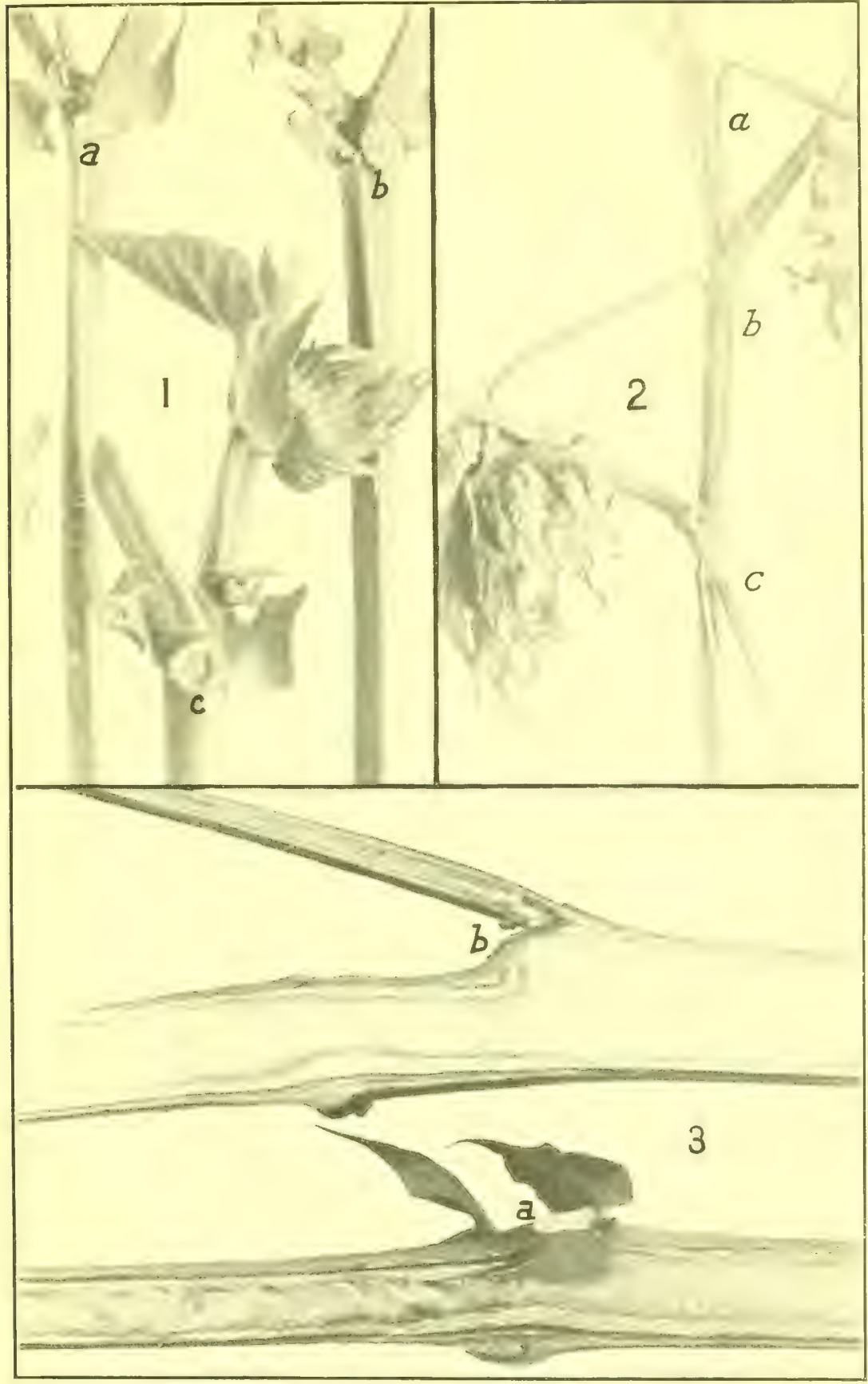

SHEDding AND RETENTION OF Forms ON THE COTTON PLANT.

Fig. 1.- $a, b, c$, Normal sears left hy shed forms. Fig, 2,- $a, b, c$, Ahizormal sears with forms retatined. Fig, 3, - $a$, Longitudinal section through base of shed form: $b$, Longitulinal section through base of retained form. Fig. 1, natural size; fig. "2, smewhat reduced; fig. 3, enlarged two diameters. (After Hinds.) 

stages of which 2,604 were killed by climate, 1,746 by predator's, and 2,897 by parasites. In other words the average percentage of control by all kinds of insects is 31.61 per cent, or 11.93 per cent by predators and 19.68 per cent by parasites. It appear's that July and $A$ ugust are usually the best months for parasite control in hanging squares.

TABLE IV.- Honthly mortality of the boll weevil due to insects in hanging bolls.

\begin{tabular}{|c|c|c|c|c|c|c|c|c|c|}
\hline \multirow{2}{*}{ Months. } & \multirow{2}{*}{$\begin{array}{l}\text { Bolls } \\
\text { exam- } \\
\text { ined. }\end{array}$} & \multirow{2}{*}{$\begin{array}{l}\text { Stages } \\
\text { found. }\end{array}$} & \multicolumn{3}{|c|}{ Stages killed by- } & \multicolumn{4}{|c|}{ Percentage of stages killed. } \\
\hline & & & $\begin{array}{l}\text { Cli- } \\
\text { mate. }\end{array}$ & $\begin{array}{l}\text { Preda } \\
\text { tors. }\end{array}$ & $\begin{array}{l}\text { Para- } \\
\text { sites. }\end{array}$ & Total. & $\begin{array}{l}\text { Preda- } \\
\text { tors. }\end{array}$ & $\begin{array}{l}\text { Para- } \\
\text { sites. }\end{array}$ & $\begin{array}{l}\text { All in- } \\
\text { sects. }\end{array}$ \\
\hline 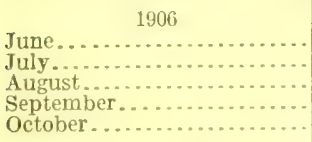 & $\begin{array}{r}145 \\
2,947 \\
23,451 \\
6,210 \\
399\end{array}$ & $\begin{array}{r}0 \\
290 \\
2,977 \\
1,555 \\
147\end{array}$ & $\begin{array}{r}0 \\
28 \\
416 \\
198 \\
21\end{array}$ & $\begin{array}{r}0 \\
14 \\
742 \\
188 \\
11\end{array}$ & $\begin{array}{r}0 \\
0 \\
19 \\
23 \\
3\end{array}$ & $\begin{array}{r}0.0 \\
14.5 \\
39.5 \\
26.3 \\
23.8\end{array}$ & $\begin{array}{r}0.0 \\
4.8 \\
24.9 \\
12.1 \\
7.5\end{array}$ & $\begin{array}{r}0.0 \\
0 \\
.6 \\
1.5 \\
2.0\end{array}$ & $\begin{array}{r}0.0 \\
4.8 \\
25.5 \\
13.6 \\
9.5\end{array}$ \\
\hline 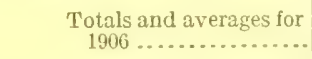 & 33,155 & 4,969 & 663 & 955 & 45 & 35.9 & 21.9 & 0.9 & 22.8 \\
\hline 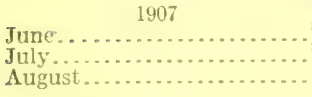 & $\begin{array}{r}50 \\
50 \\
1,272\end{array}$ & $\begin{array}{r}5 \\
7 \\
330\end{array}$ & $\begin{array}{r}1 \\
1 \\
115\end{array}$ & $\begin{array}{r}0 \\
0 \\
51\end{array}$ & $\begin{array}{l}1 \\
0 \\
4\end{array}$ & $\begin{array}{l}40.0 \\
14.3 \\
51.5\end{array}$ & $\begin{array}{r}0 \\
15.4\end{array}$ & $\begin{array}{r}20.0 \\
0 \\
1.2\end{array}$ & $\begin{array}{r}20.0 \\
0 \\
16.0\end{array}$ \\
\hline $\begin{array}{r}\text { Totals and averages for } \\
1907 \ldots \ldots . . .\end{array}$ & 1,372 & 342 & 117 & 51 & 5 & 50.6 & 14.9 & 1.4 & 16.3 \\
\hline 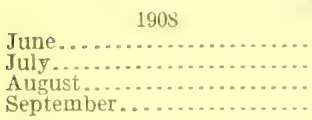 & $\begin{array}{r}2,227 \\
4,450 \\
1,123 \\
933\end{array}$ & $\begin{array}{r}238 \\
405 \\
200 \\
95\end{array}$ & $\begin{array}{r}34 \\
125 \\
22 \\
8\end{array}$ & $\begin{array}{r}6 \\
26 \\
20 \\
4\end{array}$ & $\begin{array}{r}21 \\
13 \\
0 \\
1\end{array}$ & $\begin{array}{l}29.7 \\
40.5 \\
21.0 \\
13.2\end{array}$ & $\begin{array}{r}2.5 \\
6.4 \\
10.0 \\
4.1\end{array}$ & $\begin{array}{r}8.8 \\
3.2 \\
0 \\
1.0\end{array}$ & $\begin{array}{r}11.3 \\
9.6 \\
10.0 \\
5.1\end{array}$ \\
\hline $\begin{array}{r}\text { Totals and averages for } \\
1908 \ldots . . .\end{array}$ & 8,733 & 941 & 189 & 56 & 35 & 29.7 & 5.9 & 3.7 & 9.6 \\
\hline 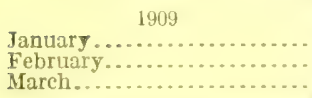 & $\begin{array}{r}1,616 \\
716 \\
608\end{array}$ & $\begin{array}{r}402 \\
115 \\
56\end{array}$ & $\begin{array}{r}133 \\
12 \\
14\end{array}$ & $\begin{array}{l}25 \\
49 \\
12\end{array}$ & $\begin{array}{r}11 \\
2 \\
1\end{array}$ & $\begin{array}{l}42.0 \\
51.7 \\
43.2\end{array}$ & $\begin{array}{r}6.2 \\
42.6 \\
21.4\end{array}$ & $\begin{array}{l}2.7 \\
1.7 \\
1.7\end{array}$ & $\begin{array}{r}8.9 \\
44.3 \\
23.1\end{array}$ \\
\hline $\begin{array}{l}\text { Totals and averages for } \\
1909 . \ldots \ldots \ldots \ldots . . . . . . . .\end{array}$ & 2,940 & 573 & 159 & 86 & 14 & 45.2 & 15.0 & 2.4 & 17.4 \\
\hline
\end{tabular}

In fallen bolls the principal insect work is that accomplished by predatory insects, and the total insect control has been less than the climatic control except in the year 1906. Table IV covers an examination of 6,825 weevil stages, of which 1,128 were killed by climate, 1,148 by predators, and only 99 by parasites. This means that 18.2 per cent of all the stages were killed by insects, or 16.8 per cent by predators and 1.4 per cent by parasites. In this class of infested forms it is also noticeable that the principal work by the predators is accomplished during the month of August.

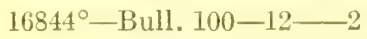


TABLE V.-Monthly mortality of the boll weevil due to insects in hanging bolls.

\begin{tabular}{|c|c|c|c|c|c|c|c|c|c|}
\hline \multirow{2}{*}{ Month. } & \multirow{2}{*}{$\begin{array}{l}\text { Bolls } \\
\text { exam- } \\
\text { ined. }\end{array}$} & \multirow{2}{*}{$\begin{array}{l}\text { Stages } \\
\text { found. }\end{array}$} & \multicolumn{3}{|c|}{ Stages killed by- } & \multicolumn{4}{|c|}{ P'ercentage of stages killed. } \\
\hline & & & $\begin{array}{l}\text { Cli- } \\
\text { mate. }\end{array}$ & $\begin{array}{l}\text { Pred- } \\
\text { ators. }\end{array}$ & $\begin{array}{l}\text { Para- } \\
\text { siles. }\end{array}$ & Total. & $\begin{array}{l}\text { Pred- } \\
\text { ators. }\end{array}$ & $\begin{array}{l}\text { Para- } \\
\text { sites. }\end{array}$ & All \\
\hline 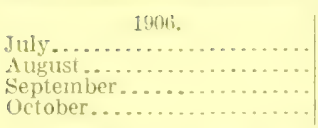 & $\begin{array}{r}434 \\
8.762 \\
4,22 ! \\
3,623\end{array}$ & $\begin{array}{l}22 \\
2,444 \\
1,627 \\
1,359\end{array}$ & $\begin{array}{r}2 \\
2 \times 1 \\
130 \\
186\end{array}$ & $\begin{array}{r}3 \\
840 \\
2 \div 2 \\
216\end{array}$ & $\begin{array}{r}0 \\
155 \\
101 \\
66\end{array}$ & $\begin{array}{l}32.6 \\
31.7 \\
32.1 \\
38.2\end{array}$ & $\begin{array}{l}13.6 \\
13.9 \\
17.9 \\
19.6\end{array}$ & $\begin{array}{l}0.0 \\
6.3 \\
6.2 \\
4.8\end{array}$ & $\begin{array}{l}13.6 \\
21.2 \\
21.1 \\
24.4\end{array}$ \\
\hline $\begin{array}{r}\text { Totals and averages for } \\
1906 . . . . . . . . . . . .\end{array}$ & 17,049 & 5,452 & 599 & 901 & 322 & 33.4 & 16.5 & 5.9 & 22.4 \\
\hline $\begin{array}{l}1908 . \\
\text { July................ } \\
\text { August........ }\end{array}$ & $\begin{array}{l}460 \\
683\end{array}$ & $\begin{array}{r}38 \\
393\end{array}$ & $\stackrel{2}{3 \tilde{3}}$ & $\begin{array}{r}0 \\
13\end{array}$ & $\begin{array}{r}0 \\
50\end{array}$ & $\begin{array}{r}5.3 \\
25.0\end{array}$ & $\begin{array}{r}0 \\
3.6\end{array}$ & $\begin{array}{r}0 \\
12.5\end{array}$ & $\begin{array}{r}0 \\
16.1\end{array}$ \\
\hline $\begin{array}{r}\text { Totals and a verages for } \\
1907 \ldots \ldots \ldots\end{array}$ & 1,143 & 431 & 37 & 13 & 50 & 23.2 & 3.0 & 11.6 & 14.6 \\
\hline 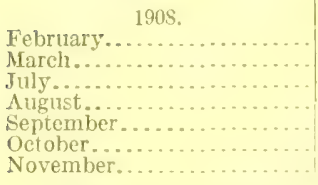 & $\begin{array}{r}12,451 \\
1,239 \\
2,132 \\
720 \\
664 \\
433 \\
519\end{array}$ & $\begin{array}{r}515 \\
22 \\
192 \\
191 \\
83 \\
262 \\
274\end{array}$ & $\begin{array}{r}424 \\
21 \\
6.3 \\
23 \\
7 \\
36 \\
134\end{array}$ & $\begin{array}{r}0 \\
0 \\
37 \\
7 \\
17 \\
11 \\
1\end{array}$ & $\begin{array}{r}54 \\
1 \\
58 \\
22 \\
2 \\
5 \\
8\end{array}$ & $\begin{array}{r}92.8 \\
100.0 \\
32.0 \\
27.2 \\
31.3 \\
19.8 \\
52.2\end{array}$ & $\begin{array}{r}0 \\
0 \\
7.5 \\
3.6 \\
20.4 \\
4.2 \\
.3\end{array}$ & $\begin{array}{r}10.5 \\
4.5 \\
11.7 \\
11.5 \\
2.4 \\
1.9 \\
2.9\end{array}$ & $\begin{array}{r}10.5 \\
4.5 \\
19.1 \\
15.1 \\
22.8 \\
6.1 \\
3.2\end{array}$ \\
\hline $\begin{array}{l}\text { Totals and averages for } \\
1908 . . . . . . . . . . . . . .\end{array}$ & $18,15 i$ & $1.839 \mid$ & 708 & 73 & 150 & 50.6 & 3.9 & 8.11 & 12.0 \\
\hline 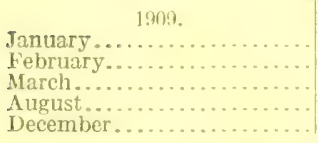 & $\begin{array}{r}3,941 \\
430 \\
653 \\
365 \\
2,148\end{array}$ & $\begin{array}{r}857 \\
35 \\
81 \\
42 \\
519\end{array}$ & $\begin{array}{r}335 \\
13 \\
16 \\
1 \\
217\end{array}$ & $\begin{array}{r}35 \\
11 \\
16 \\
2 \\
13\end{array}$ & $\begin{array}{r}43 \\
0 \\
0 \\
0 \\
11\end{array}$ & \begin{tabular}{r|}
48.5 \\
68.6 \\
39.5 \\
8.1 \\
46.4
\end{tabular} & $\begin{array}{r}4.4 \\
31.4 \\
19.7 \\
5.4 \\
25.0\end{array}$ & \begin{tabular}{r|r}
5.0 \\
0 \\
0 \\
0 \\
2.1
\end{tabular} & $\begin{array}{r}9.4 \\
31.4 \\
19.7 \\
5.4 \\
27.1\end{array}$ \\
\hline $\begin{array}{l}\text { Totals and averages for } \\
1909 \ldots \ldots \ldots \ldots \ldots . . . . .\end{array}$ & $7,537 \mid$ & 1.534 & 582 & so & 54 & 46.71 & 5.2 & 3.51 & 8.7 \\
\hline
\end{tabular}


Table $V$ was based, as may he seen, upon the examination of 9,256 weevil stages, of which 1,926 were killed hy climate, 1,067 hy predators, and 576 by parasites. In other words, tho insect enemies killed 17.74 per cent, of which 11.52 per cent were killed by predators and 6.2.2 per eent by parasites. July and August are the principal months for attack upon hanging bolls.

Summarizing the four preceding tables, Table VI is presented to show the monthly rate of mortality in all classes of infested forms.

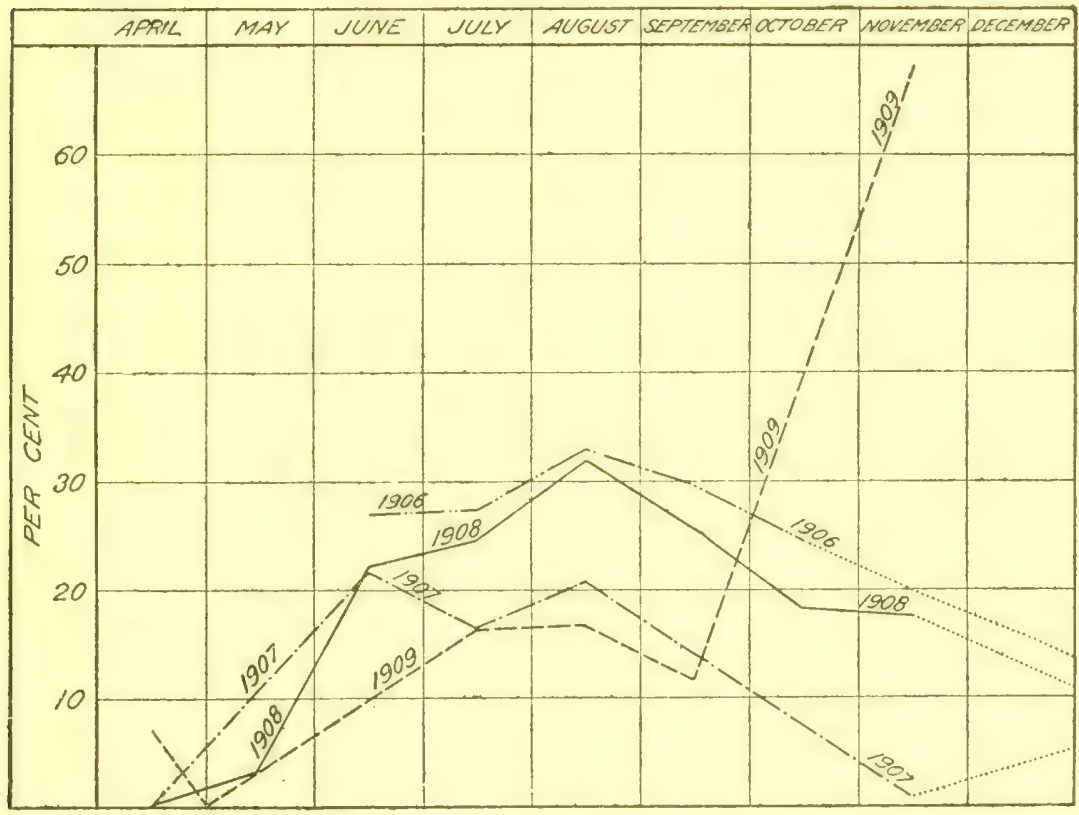

FIG. 1.-Diagram illustrating the monthly pereentage of mortality of immature boll weevils due to insect enemies. (Original.)

It will be noticed that in cach year certain months have been omitted and it must be explained that the reason therefor has been the necessity of making these examinations only when other work was not pressing. An analysis of this table is more readily made by reference to the accompanying diagram (fig. 1), which demonstrates conclusirely that August is the month during which the insect enemies of the boll weevil are most active. 
'IABLE VI.-Monthly rate of mortality of the boll weevil in infested forms of all classes

\begin{tabular}{|c|c|c|c|c|c|c|c|}
\hline \multirow{2}{*}{ Month. } & \multirow{2}{*}{$\begin{array}{l}\text { Forms } \\
\text { exam- } \\
\text { ined. }\end{array}$} & \multirow{2}{*}{$\begin{array}{l}\text { Stages } \\
\text { found. }\end{array}$} & \multicolumn{5}{|c|}{ Percentage of stages killed by- } \\
\hline & & & $\begin{array}{c}\text { All } \\
\text { causes. }\end{array}$ & Climate. & $\begin{array}{l}\text { Preda- } \\
\text { tors. }\end{array}$ & $\begin{array}{l}\text { Para. } \\
\text { sites. }\end{array}$ & $\begin{array}{c}\text { All } \\
\text { insects. }\end{array}$ \\
\hline 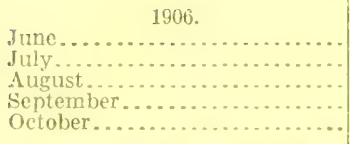 & $\begin{array}{r}4,621 \\
11,120 \\
55,686 \\
23,175 \\
6,042\end{array}$ & $\begin{array}{r}3,831 \\
5,111 \\
19,173 \\
9,832 \\
2,126\end{array}$ & $\begin{array}{l}73.80 \\
61.67 \\
54.64 \\
50.40 \\
44.12\end{array}$ & $\begin{array}{l}46.90 \\
34.29 \\
21.19 \\
20.80 \\
19.56\end{array}$ & $\begin{array}{l}23.80 \\
21.83 \\
29.32 \\
24.14 \\
19.75\end{array}$ & $\begin{array}{l}3.10 \\
5.53 \\
3.61 \\
5.43 \\
4.79\end{array}$ & $\begin{array}{l}26.90 \\
27.36 \\
32.93 \\
29.57 \\
24.54\end{array}$ \\
\hline Totals and averages for 1906 & 100,644 & 40,073 & 55.81 & 25.15 & 26.31 & 4.31 & 30.62 \\
\hline 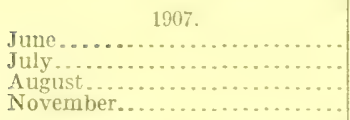 & $\begin{array}{r}2,274 \\
6,658 \\
12,898 \\
150\end{array}$ & $\begin{array}{r}1,354 \\
4,166 \\
7,792 \\
93\end{array}$ & $\begin{array}{l}48.67 \\
36.55 \\
64.19 \\
97.70\end{array}$ & $\begin{array}{l}27.03 \\
20.11 \\
33.39 \\
96.70\end{array}$ & $\begin{array}{r}14.99 \\
10.77 \\
20.67 \\
0\end{array}$ & $\begin{array}{r}6.64 \\
5.66 \\
10.12 \\
1.00\end{array}$ & $\begin{array}{r}21.63 \\
16.43 \\
30.79 \\
1.00\end{array}$ \\
\hline Totals and averages for 1907 & 21,980 & 13,405 & 54.27 & 29.06 & 16.88 & 8.32 & 25.20 \\
\hline 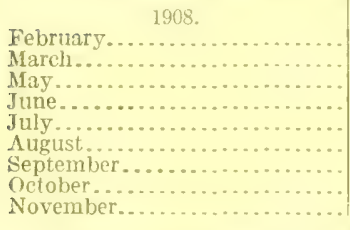 & $\begin{array}{r}12,451 \\
1,329 \\
100 \\
10,035 \\
16,974 \\
5,177 \\
12,708 \\
11,302 \\
2,248\end{array}$ & $\begin{array}{r}515 \\
22 \\
56 \\
5,523 \\
7,764 \\
2,441 \\
6,415 \\
6.157 \\
653\end{array}$ & $\begin{array}{r}92.81 \\
100.00 \\
10.70 \\
43.81 \\
45.63 \\
61.53 \\
42.29 \\
33.92 \\
50.53\end{array}$ & $\begin{array}{r}82.33 \\
95.50 \\
7.20 \\
15.78 \\
20.99 \\
29.79 \\
16.66 \\
15.57 \\
35.52\end{array}$ & $\begin{array}{r}0 \\
0 \\
0 \\
15.78 \\
14.73 \\
16.87 \\
17.06 \\
5.73 \\
3.06\end{array}$ & $\begin{array}{r}10.48 \\
4.50 \\
3.50 \\
6.24 \\
9.90 \\
14.91 \\
8.55 \\
12.61 \\
14.70\end{array}$ & $\begin{array}{r}10.48 \\
4.50 \\
3.50 \\
22.02 \\
24.63 \\
31.75 \\
25.61 \\
18.34 \\
17.76\end{array}$ \\
\hline Totals and a verages for 1908 & 72,231 & 29,546 & 44.34 & 21.21 & 13.12 & 10.00 & 23.12 \\
\hline 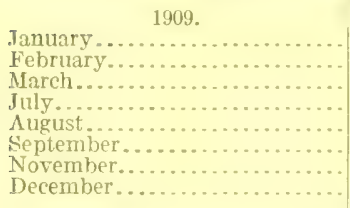 & $\begin{array}{l}5,687 \\
1,146 \\
1,261 \\
8,307 \\
7,162 \\
1,495 \\
136 \\
2,663\end{array}$ & $\begin{array}{r}1,285 \\
150 \\
137 \\
4.717 \\
3,764 \\
860 \\
52 \\
685\end{array}$ & $\begin{array}{r}45.52 \\
58.00 \\
43.06 \\
15.36 \\
37.32 \\
21.25 \\
100.00 \\
52.32\end{array}$ & $\begin{array}{l}36.42 \\
16.66 \\
21.89 \\
28.70 \\
20.64 \\
9.30 \\
32.00 \\
38.37\end{array}$ & $\begin{array}{r}4.90 \\
40.00 \\
20.43 \\
11.19 \\
13.33 \\
3.02 \\
0 \\
2.03\end{array}$ & $\begin{array}{r}4.20 \\
1.33 \\
.72 \\
5.25 \\
3.34 \\
8.95 \\
68.00 \\
11.91\end{array}$ & $\begin{array}{r}9.10 \\
41.33 \\
21.15 \\
16.34 \\
16.67 \\
11.97 \\
68.00 \\
13.94\end{array}$ \\
\hline Totals and a verages for 1009 & 27,857 & 11,653 & 41.73 & 25.84 & 10.56 & 5.32 & 15.88 \\
\hline
\end{tabular}

\section{A GEOGRAPIIC STUDY OF THE STATISTICS OF INSECT CONTROL.}

A study of these same statistics, when arranged to show the insect control by fiates, has giren much interesting light upon the subject of the control of the weevil.

In fallen squares we find an average for total insect control of 26.8 per cent in Oklahoma, 25.9 per cent in Mississippi, 24.5 per cent in 'Texas, 20.6 per cent in Louisiana, and 12.5 per cent in Arkansas. Analyaing these firures from another standpoint, we find that the State of Mississippi learls in parasite control with 14.27 per cent, Oklahoma standing next with 4.71 per cent, Texas with 3.9 per cent, Louisiana with 2.52 per cent, and Arkansas with 0.71 per cent. The relative rank of the States for predatory control is quite different. Clahoma leads with 22.16 per cent, Texas comes next with 20.6 per cent, Louisiana with 18.1 per cent, Arkansas with 11.82 per cent, and Mississippi with 11.63 per cent. In climatic control Texas leads 
with 37.9 per cent, Oklahoma comes next with 30.8 per cent, Arkansas with 25.65 per eent, Louisiana with 12.5 per eent, and Mlississippi with 11.7 per eent. Thus it may be secn that the dry, prairie sitates of Texas and Oklahoma lead in the climatic and predatory control of the weevil and also in the total amount of control, and that the climatie control in each of these sitates is greater than the total insect control. This latter fact is also true of Arkansas. In Louisiana and Mississippi, States which are naturally more hmid, the climate has less influence and the oreater proportion of the control is by the insect enemies.

In hanging squares the conditions are entirely reversed. It is noticeable that () klahoma leads in parasit ism with an aroratere of 31.74 per cent, Texas averages 26.6 per cent, Arkansas 24.16 per cent, Mississipi 21.2 per cent, and Louisiana 12.07 per cent. In predatory control Louisiana leads with 12.9 per cent, Texas comes next with 10.9

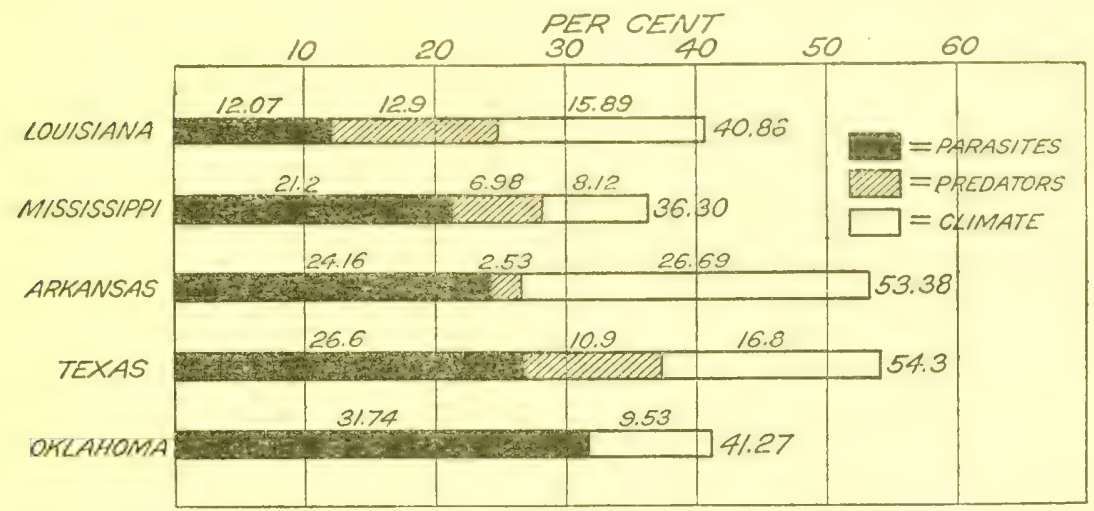

FIG. 2,-Diagram illustrating the arerage climatic and insect control of the immature boll weevils during 1906, 1907, 1908, and 1909, in hanging squares. (Original.)

per cent, Mississippi with $6.9 \mathrm{~s}$ per cent, and Arkansas with 2.58 per cent. We have no record of predatory control in OkJahoma. In all five States insect control in hanging squares is greater than climatic control. With regard to climatic control Arkansas leads with 26.69 per cent, Texas has 16.8 per cent, Louisiana 15.89 per eent, Oklahoma 9.53 per eent, and Mississippi 8.12 per cent. These statisties are graphically shown in figures 2 and 3.

A brief comparison of the condition in hanging and fallen squares will show that the States of Texas and Oklahoma have a higher arerage pereentage of control from all factors in fallen squares than in the hanging squares; the States of Louisiana and Arkansas have a higher average pereentage of control from all factors in hanging squares than in fallen squares, and in the State of Mississippi the difference is rery slight, although in favor of the fallen squares. 'This illustrates the 
diffeulty of giving any single recommendation for the rontrol of the boll weevil which would apply to all regions. This point will be brought out more fully in other sections of this bulletin.

\section{A STUDY OF THE SHARE OF INSECT CONTROL IN THE MORTALITY OF} IMMATURE BOLI, WEEVILS.

The condensed tables which have been presented are likely to give the impression that the parasite control of the werevil is on an arerage very low, but it must be remembered that the examinations have been made in all parts of the infested region whether the weevil has been present 17 years or only a few months, and whether the weevil damage amounts to less than 1 per cent of the crop or to almost 100

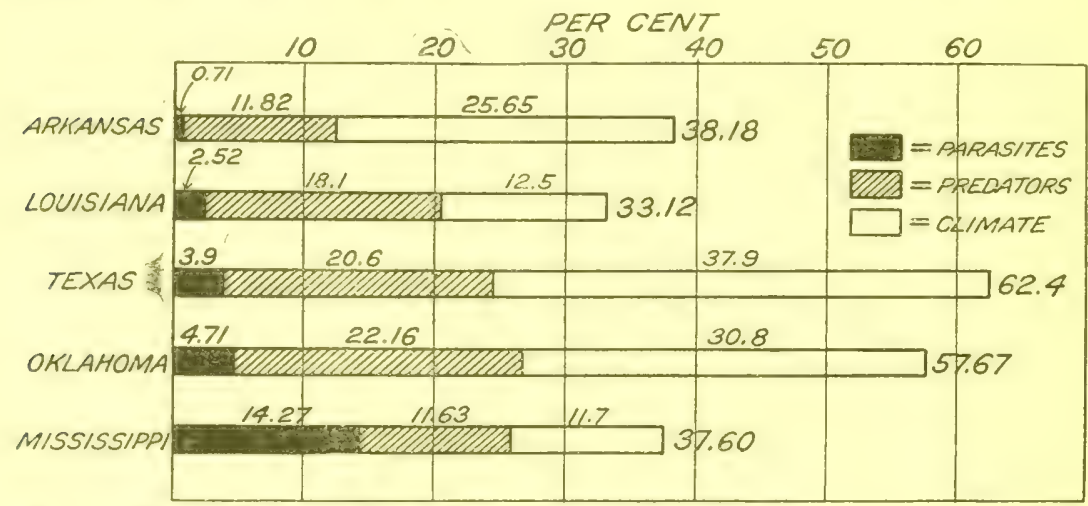

Fli. 3,-1)iagrum illuctrating the average climatic and insect control of the immature boll weevils during $1906,1907,1908$, and 1909 , in fallen squares. (Original.)

per cent. 'This great difference in the sources of the material examined has necessarily lowered the average mortality to its minimum. The following records show some of the cases of very high mortality due to parasites:

Highest records of parasitism of the boll weevil.

IN FALLEN SQUARES.

\begin{tabular}{|c|c|c|c|}
\hline Locality. & Date. & $\begin{array}{l}\text { Number } \\
\text { of stages. }\end{array}$ & $\begin{array}{l}\text { Percent- } \\
\text { age of } \\
\text { parasit- } \\
\text { ism. }\end{array}$ \\
\hline 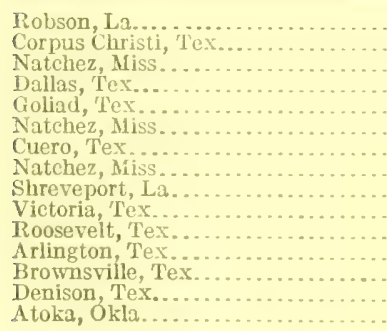 & 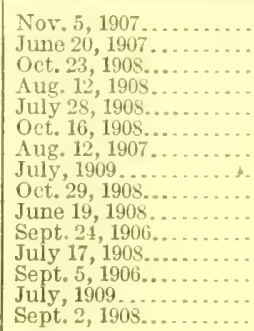 & $\begin{array}{r}53 \\
92 \\
157 \\
18 \\
114 \\
230 \\
105 \\
200 \\
624 \\
513 \\
69 \\
382 \\
1,177 \\
494 \\
100\end{array}$ & $\begin{array}{l}77.36 \\
36.95 \\
28.6 \\
27.78 \\
26.63 \\
21.7 \\
19.04 \\
18.5 \\
15.8 \\
14.5 \\
14.4 \\
13.35 \\
12.4 \\
8.5 \\
8.0\end{array}$ \\
\hline
\end{tabular}


Highest records of parasitism of the boll weevil-Continued.

IN HANGING SQUARES.

\begin{tabular}{|c|c|c|c|}
\hline Locality. & Date. & $\begin{array}{l}\text { Number } \\
\text { of stages. }\end{array}$ & $\begin{array}{l}\text { Pereent- } \\
\text { age of } \\
\text { parasit- } \\
\text { 1sm. }\end{array}$ \\
\hline 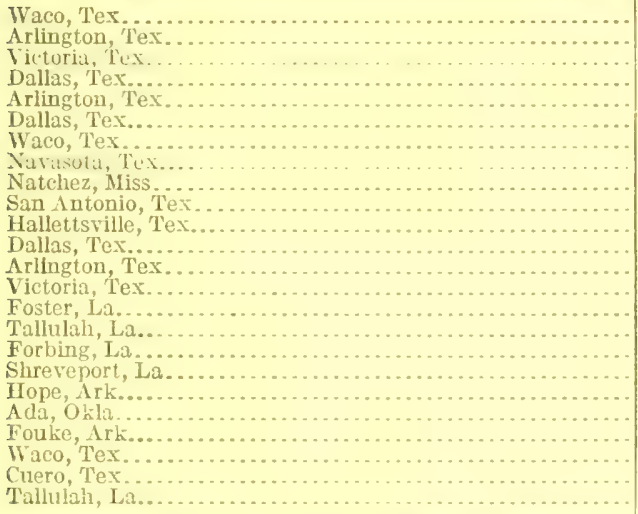 & 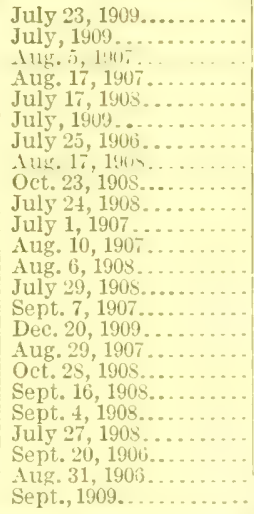 & $\begin{array}{r}39 \\
55 \\
-11 \\
52 \\
51 \\
57 \\
99 \\
22 \\
82 \\
29 \\
19 \\
193 \\
260 \\
140 \\
22 \\
85 \\
41 \\
37 \\
69 \\
63 \\
251 \\
109 \\
347 \\
495\end{array}$ & $\begin{array}{l}66.6 \\
63.63 \\
61.5 \\
59.7 \\
56.86 \\
52.63 \\
52.6 \\
51.7 \\
51.3 \\
48.27 \\
47.3 \\
47.15 \\
46.92 \\
45.71 \\
45.47 \\
44.7 \\
43.9 \\
37.84 \\
33.33 \\
31.74 \\
26.05 \\
23.8 \\
21.3 \\
15.35\end{array}$ \\
\hline
\end{tabular}

IN FALLEN BOLLS.

\begin{tabular}{|c|c|c|}
\hline 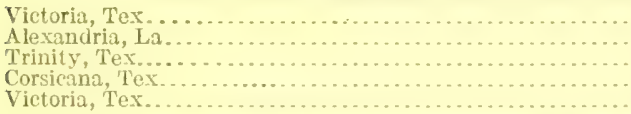 & $\begin{array}{l}\text { June } 17-29,1908 \ldots \ldots . \\
\text { July } 29,1905 \ldots \ldots \ldots . \\
\text { Aug. } 24,1907 \ldots \ldots \ldots . \\
\text { Sept. } 18,1906 \ldots \ldots \ldots \ldots \\
\text { Jan., } 1909 . \ldots \ldots \ldots \ldots \ldots\end{array}$ & $\begin{array}{l}97 \\
22 \\
12 \\
31 \\
87\end{array}$ \\
\hline
\end{tabular}

IN IIANGING BOLLS.

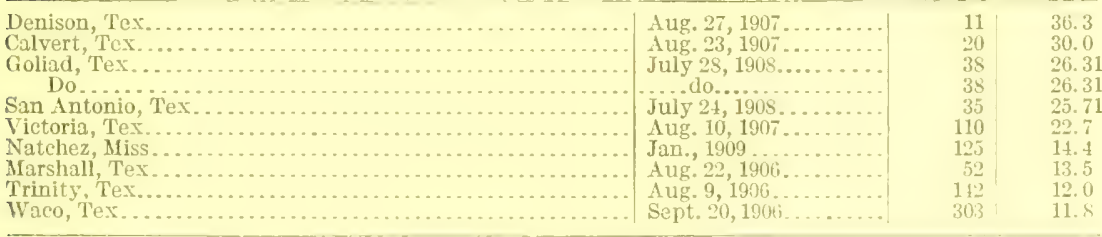

These records give merely the highest percentages for each State in each year. There are many other records which might be included between the above figures.

Highest records of total insect control of the boll weevil.

IN FALLEN SQUARES.

\begin{tabular}{|c|c|c|c|}
\hline Locality. & Date. & $\begin{array}{l}\text { Number } \\
\text { of stages. }\end{array}$ & $\begin{array}{l}\text { Percent- } \\
\text { age of } \\
\text { Insect } \\
\text { control. }\end{array}$ \\
\hline 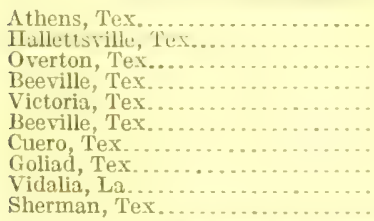 & $\begin{array}{l}\text { Aug. 1, 1907.. } \\
\text { Aug. 1, 130... } \\
\text { Aug. 1, 1906.. } \\
\text { July 29, } 1908 . \\
\text { Sept. 1, } 1906 . . \\
\text { June 20, } 1908 . \\
\text { Aug. } 28,1908 . \\
\text { Sept. } 15,1908 . \\
\text { July, } 1909 . . .\end{array}$ & $\begin{array}{r}255 \\
100 \\
197 \\
1,310 \\
375 \\
678 \\
549 \\
114 \\
142 \\
171\end{array}$ & 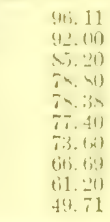 \\
\hline
\end{tabular}


Highest records of total insect control of the boll weevil-Continued.

IN HANGING SQUARES.

\begin{tabular}{|c|c|c|c|}
\hline Locality. & Date. & $\begin{array}{l}\text { Number } \\
\text { of stages. }\end{array}$ & $\begin{array}{l}\text { Percent- } \\
\text { age of } \\
\text { insect } \\
\text { control. }\end{array}$ \\
\hline 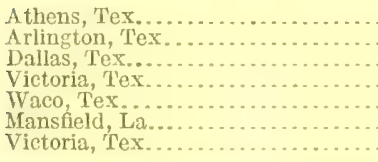 & 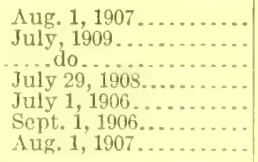 & $\begin{array}{r}75 \\
55 \\
57 \\
87 \\
99 \\
244 \\
253\end{array}$ & $\begin{array}{l}84.00 \\
75.44 \\
69.99 \\
58.54 \\
56.56 \\
50.90 \\
50.00\end{array}$ \\
\hline
\end{tabular}

IN IAANGING BOLLS.

\begin{tabular}{|c|c|c|c|}
\hline 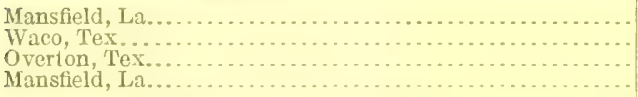 & $\begin{array}{l}\text { Sept. } 29,1906 \ldots \ldots \ldots . \\
\text { Aug. } 1,1906 \ldots \ldots \ldots . \\
\text { Sept. } 24,1906 \ldots \ldots \ldots .\end{array}$ & $\begin{array}{r}145 \\
421 \\
89 \\
479\end{array}$ & $\begin{array}{l}58.30 \\
42.04 \\
40.50 \\
33.00\end{array}$ \\
\hline
\end{tabular}

THE CORRECT BASIS FOR COMPARISON OF MORTALITY STATISTICS.

As has been explained, the examinations have been made from various sources. It is therefore necessary to arrive at some true basis for the comparison of these data before an exact knowledge of the conditions existing can be obtained. The first mortality of the weevil is that due to proliferation. Dr. Hinds, in Bulletin 59 of this bureau, has shown that the average mortality of weeril stages in squares from proliferation is 13.5 per cent and that the average mortality in bolls is 6.3 per cent. In the absence of further data these two percentages are used as a basis for obtaining the weighted average mortality.

As nearly as the proportion can be estimated throughout the entire season, 15 per cent of the weevil stages are to be found in bolls and 5 per cent in hanging forms. Whether these arbitrary estimates be true or not, this is the only manner in which it will be possible to compare the mortality by the different factors in the rarious years. On this basis, therefore, a series of hypothetical tables has been erected.

In order to show how the hypothetical average differs from the average obtained from the total examinations, two tables are given for cach year, the first being a table giving the actual conditions in the four classes of infested material and the second table being a hypothetical table based upon 10,000 weevil stages on the arbitrary basis of 5 per cent of the stages in hanging forms and 15 per cent of the stages in bolls. The process continues by first subtracting the mortality by proliferation and then computing the mortality from climate, predators, and parasites from the remainder. The percentages of mortality given in the total line are based upon the total of 10,000 stages. 
1906. - The data on the mortality of the weevil in 1906 may therefore be condensed and tabulated as follows:

TABLE VII.-Boll-weevil mortality in 1906.

\begin{tabular}{|c|c|c|c|c|c|c|}
\hline \multirow{2}{*}{ Class of forms. } & \multirow{2}{*}{$\begin{array}{c}\text { Number of } \\
\text { weevil } \\
\text { stages. }\end{array}$} & \multirow{2}{*}{$\begin{array}{l}\text { Percentage } \\
\text { of stages } \\
\text { alive. }\end{array}$} & \multicolumn{3}{|c|}{ Percentage of stages killed by- } & \multirow{2}{*}{$\begin{array}{l}\text { Total per- } \\
\text { centage of } \\
\text { mortality. }\end{array}$} \\
\hline & & & Climate. & Predators. & Parasites. & \\
\hline 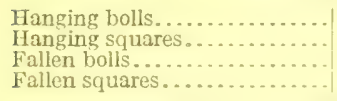 & $\begin{array}{r}5,452 \\
4,118 \\
4,969 \\
25.534\end{array}$ & $\begin{array}{l}66.59 \\
46.20 \\
64.53 \\
34.80\end{array}$ & $\begin{array}{l}10.98 \\
20.30 \\
13.34 \\
31.20\end{array}$ & $\begin{array}{l}16.52 \\
20.50 \\
19.01 \\
30.70\end{array}$ & $\begin{array}{r}5.90 \\
13.00 \\
.90 \\
3.30\end{array}$ & $\begin{array}{l}33.41 \\
53.80 \\
35.47 \\
65.20\end{array}$ \\
\hline Totals and averages..... & 40,073 & 44.19 & 25.15 & 26.31 & 4.31 & 55.81 \\
\hline
\end{tabular}

TABLE VIII.--The hypothetical or weighted average mortality of the boll wecvil, 1906.

\begin{tabular}{|c|c|c|c|c|c|c|c|c|c|c|c|c|c|}
\hline \multirow[b]{3}{*}{ Class of forms. } & \multirow{3}{*}{ 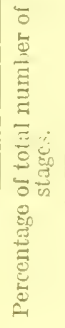 } & \multirow[b]{3}{*}{ 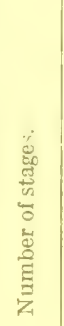 } & \multicolumn{11}{|c|}{ 1906-Mortality from- } \\
\hline & & & \multicolumn{2}{|c|}{$\begin{array}{l}\text { Prolifera- } \\
\text { tion. }\end{array}$} & \multirow[b]{2}{*}{ 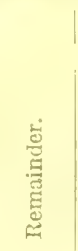 } & \multicolumn{2}{|c|}{ Climate. } & \multicolumn{2}{|c|}{ Predators. } & \multicolumn{2}{|c|}{ Parasites. } & \multicolumn{2}{|c|}{ Total. } \\
\hline & & & 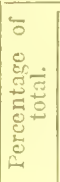 & 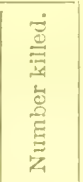 & & 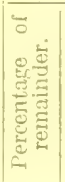 & 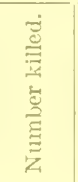 & 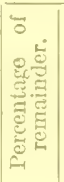 & 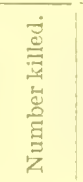 & 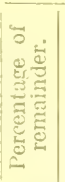 & 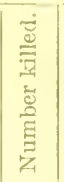 & 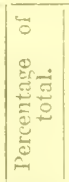 & 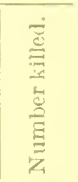 \\
\hline $\begin{array}{l}\text { Hanging bolls........ } \\
\text { Hanging squares..... }\end{array}$ & $\begin{array}{l}0.75 \\
4.25\end{array}$ & $\begin{array}{r}75 \\
425\end{array}$ & $\begin{array}{r}6.30 \\
13.50\end{array}$ & $\begin{array}{r}4.7 \\
57.4\end{array}$ & $\begin{array}{r}70.3 \\
367.6\end{array}$ & $\begin{array}{l}10.98 \\
20.30\end{array}$ & $\begin{array}{r}7.7 \\
74.6\end{array}$ & $\begin{array}{l}16.52 \\
20.50\end{array}$ & $\begin{array}{l}11.6 \\
75.4\end{array}$ & $\begin{array}{r}5.90 \\
13.00\end{array}$ & $\begin{array}{r}4.1 \\
47.8\end{array}$ & $\mid \begin{array}{l}37.4 \\
60.0\end{array}$ & $\begin{array}{r}2 S .1 \\
255.2\end{array}$ \\
\hline Total hanging.. & 5.00 & 500 & $\cdots$ & 62.1 & 437.9 & $\ldots$ & 82.3 & $\ldots \ldots$ & $87.0^{\prime}$ & $\ldots \ldots$ & $\mid 51.9$ & ..... & 283.3 \\
\hline $\begin{array}{l}\text { Fallen bolls...... } \\
\text { Fallen squares.. }\end{array}$ & $\begin{array}{l}14.25 \\
80.75\end{array}$ & $\begin{array}{l}1,425 \\
S, 0751\end{array}$ & $\begin{array}{r}6.30 \\
13.50\end{array}$ & $\begin{array}{r}90.0 \\
1,090.1\end{array}$ & $\begin{array}{l}1,335.0 \\
, 984.9\end{array}$ & $\begin{array}{l}13.34 \\
31.20\end{array}$ & $\begin{array}{r}178.1 \\
2,179.3\end{array}$ & $\begin{array}{l}19.01 \\
30.70\end{array}$ & $\begin{array}{r}253.8 \\
2,144.4\end{array}$ & $\begin{array}{r}.90 \\
3.30\end{array}$ & \begin{tabular}{|l|}
12.0 \\
230.5
\end{tabular} & $\begin{array}{l}37.4 \\
69.8\end{array}$ & $\begin{array}{r}533.9 \\
5.644 .3\end{array}$ \\
\hline Total fallen.... & 95.00 & 9,500 & ..... & $\mid 1,180.1$ & $3,319.9$ & $\ldots \ldots$ & $2,357.4$ & -..... & $2,398.2$ & ..... & 242.5 & $5, \ldots$. & $6,178.2$ \\
\hline $\begin{array}{r}\text { Totals and arer- } \\
\text { ages.......... }\end{array}$ & & & & & & & & & & & & & 61.5 \\
\hline
\end{tabular}

1 Given 10,000 weeril stages.

190\%.-The mortality during 1907 was 54.27 per cent when figured from the total number of stages and total mortality, thus showing a decrease of 1.54 per cent from the mortality of 1906 figured in the same manner. The parasitism showed an increase of 4.01 per cent.

TABle IX.-Boll-weevil mortality in 1907.

\begin{tabular}{|c|c|c|c|c|c|c|}
\hline \multirow{2}{*}{ Class of forms. } & \multirow{2}{*}{$\begin{array}{l}\text { Number of } \\
\text { weevil } \\
\text { stages. }\end{array}$} & \multirow{2}{*}{$\begin{array}{c}\text { Percentage } \\
\text { of stages } \\
\text { alive. }\end{array}$} & \multicolumn{3}{|c|}{ Pereentage of stages killed by-- } & \multirow{2}{*}{$\begin{array}{l}\text { Total per- } \\
\text { centage of } \\
\text { mortality. }\end{array}$} \\
\hline & & & Climate. & Predators. & Parasites. & \\
\hline $\begin{array}{l}\text { Hanging bolls................. } \\
\text { Hanging squares............. } \\
\text { Fallen bolls ................ } \\
\text { Fallen squares. . . . }\end{array}$ & $\begin{array}{r}431 \\
2,612 \\
342 \\
10,020\end{array}$ & $\begin{array}{l}76.80 \\
51.40 \\
49.42 \\
42.90\end{array}$ & $\begin{array}{r}8.58 \\
14.50 \\
31.28 \\
33.50\end{array}$ & $\begin{array}{r}3.02 \\
7.50 \\
14.91 \\
19.90\end{array}$ & $\begin{array}{r}11.60 \\
26.60 \\
1.46 \\
3.70\end{array}$ & $\begin{array}{l}23.20 \\
48.60 \\
50.58 \\
57.10\end{array}$ \\
\hline Totals and arerages.... & 13,405 & 45.73 & 29.06 & 16.88 & 8.32 & 54.27 \\
\hline
\end{tabular}


Following the plan adopted for the 1906 records these figures may be weighted for comparison with the earlier records.

'TABLE X.-The hypothetical or weighted average mortality of the boll weevil in $190 \% .1$

\begin{tabular}{|c|c|c|c|c|c|c|c|c|c|c|c|c|c|}
\hline \multirow[b]{3}{*}{ Class of forms. } & \multirow{3}{*}{ 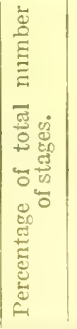 } & \multirow[b]{3}{*}{ 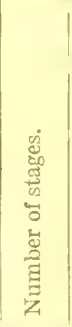 } & \multicolumn{11}{|c|}{ 1907-Mortality from - } \\
\hline & & & \multicolumn{2}{|c|}{$\begin{array}{l}\text { Prolifer- } \\
\text { ation. }\end{array}$} & \multicolumn{3}{|c|}{ Climate. } & \multicolumn{2}{|c|}{ Predators. } & \multicolumn{2}{|c|}{ Parasites. } & \multicolumn{2}{|c|}{ Total. } \\
\hline & & & 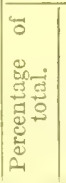 & 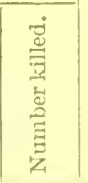 & 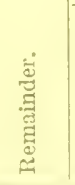 & 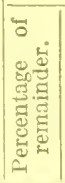 & 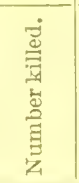 & 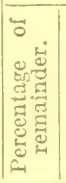 & 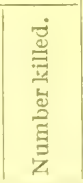 & 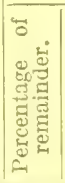 & 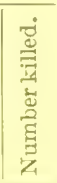 & 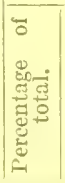 & 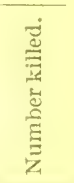 \\
\hline $\begin{array}{l}\text { Hanging bolls. . . . . . } \\
\text { Hanging squares.... }\end{array}$ & $\begin{array}{l}0.75 ! \\
4.25\end{array}$ & $\begin{array}{r}75 \\
425\end{array}$ & $\begin{array}{r}6.30 \\
13.50\end{array}$ & $\begin{array}{r}4.7 \\
57.4\end{array}$ & $\begin{array}{r}70.3 \\
367.6\end{array}$ & $\mid \begin{array}{r}8.5 s \\
14.50\end{array}$ & $\begin{array}{r}6.0 \\
53.3\end{array}$ & $\begin{array}{l}3.02 \\
7.50\end{array}$ & $\begin{array}{r}2.1 \\
27.7\end{array}$ & $\begin{array}{l}11.60 \\
26.60\end{array}$ & $\begin{array}{r}8.1 \\
97.8\end{array}$ & 29.70 & $\begin{array}{r}20.9 \\
236.2\end{array}$ \\
\hline Total hanging . - & 5.00 & 500 & $\ldots \ldots$ & 62.1 & 437.9 & $|-\ldots|$ & 59.3 & & $\overline{29.8}$ & & 105.9 & $\mid \ldots \ldots$ & 257.1 \\
\hline $\begin{array}{l}\text { Fallen bolls....... } \\
\text { Fallen squares... }\end{array}$ & $\begin{array}{l}14.25 \\
80.75\end{array}$ & $\begin{array}{l}1,425 \\
8,075\end{array}$ & $\begin{array}{r}6.30 \\
13.50\end{array}$ & $\begin{array}{r}90.0 \\
1,090.1\end{array}$ & $\begin{array}{l}1,335.0 \\
, 954.9\end{array}$ & 31.28 & $\begin{array}{r}417.6 \\
2,339.6\end{array}$ & $\begin{array}{l}14.91 \\
19.90\end{array}$ & $\begin{array}{r}199.0 \\
1,359.8\end{array}$ & $\begin{array}{l}1.46 \\
3.70\end{array}$ & $\begin{array}{r}19.5 \\
255.4\end{array}$ & $\begin{array}{l}50.90 \\
62.80\end{array}$ & $\begin{array}{r}726.1 \\
5,077.9\end{array}$ \\
\hline Total fallen..... & 95.00 & 9,500 & $\overline{\ldots . . .}$ & $1,180.1$ & 319.9 & & $\overline{2,757.2}$ & & $\overline{1,588.8}$ & $\ldots$ & 277.9 & & $5, \$ 01.0$ \\
\hline $\begin{array}{l}\text { Totals and aver- } \\
\text { ages.......... }\end{array}$ & 100.00 & 10,000 & 12.42 & $|1,2+2.1|$ & & 28.16 & $2,816.5$ & 16. 18 & $1,618.6$ & 3.83 & 353.8 & 60.61 & $6,061.1$ \\
\hline
\end{tabular}

1 Given 10,000 weevil stages.

This table shows a weighted increase of 0.89 per cent for parasites and a weighted decrease of 4 per cent for all agencies due to the falling oft in control by predators.

1908. - The mortality during 1908 was 44.34 per eent when figured from the total number of stages, the total mortality thus showing a decrease of 9.93 per cent from 1907. The parasitism showed an increase of 1.68 per cent.

TABLE XI.-Boll-weenl mortality in 1:08.

\begin{tabular}{|c|c|c|c|c|c|c|}
\hline \multirow{2}{*}{ Class of forms. } & \multirow{2}{*}{$\begin{array}{l}\text { Number of } \\
\text { weeril } \\
\text { stages. }\end{array}$} & \multirow{2}{*}{$\begin{array}{c}\text { Percentage } \\
\text { of stage's } \\
\text { alive. }\end{array}$} & \multicolumn{4}{|c|}{ I'ercentage of stages killed by- } \\
\hline & & & Climate. & Predators. & Parasites. & $\begin{array}{l}\text { All agen- } \\
\text { cies. }\end{array}$ \\
\hline 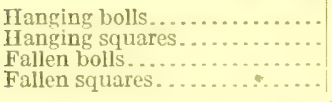 & $\begin{array}{r}1,839 \\
5,922 \\
941 \\
20,844\end{array}$ & $\begin{array}{l}49.40 \\
49.11 \\
70.25 \\
57.25\end{array}$ & $\begin{array}{l}38.48 \\
19.24 \\
20.08 \\
20.30\end{array}$ & $\begin{array}{r}3.97 \\
9.80 \\
5.95 \\
15.18\end{array}$ & $\begin{array}{r}8.15 \\
21.70 \\
3.71 \\
7.15\end{array}$ & $\begin{array}{l}50.60 \\
50.99 \\
29.75 \\
42.72\end{array}$ \\
\hline Totals and averages..... & 29,546 & 55.60 & 21.21 & 13.12 & 10.00 & 44.34 \\
\hline
\end{tabular}


Following the plan adopted for the 1906 and 1907 records these figures may be weighted for comparison with the earlier records.

TABLE XII.-The hypothetical or weighted average mortalty of the boll weevit in $1908 .{ }^{1}$

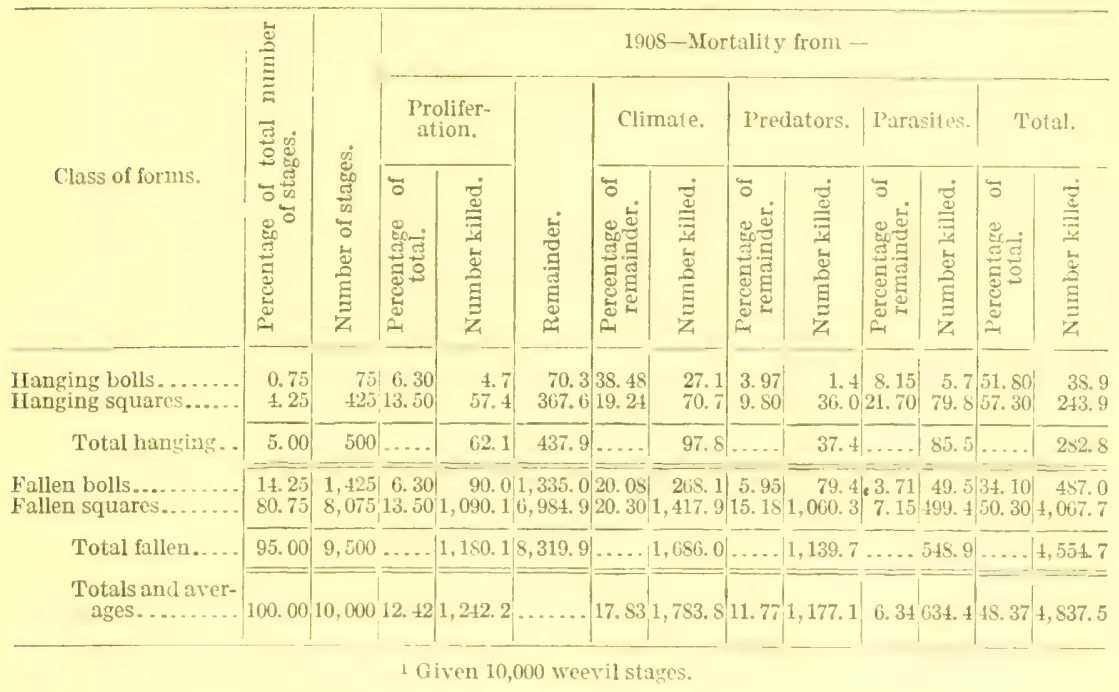

This table shows a weighted increase of 2.51 per cent for parasites and a weighted decrease of 12.24 per cent for all agencies, due to the falling off in control by both climate and predators.

1909.-The mortality during 1909 was 41.73 per cent when figured from the total number of stages, the total mortality thes showing a decrease of 2.61 per cent from 1908. The parasitism showed also a decrease amounting to 4.68 per cent.

TABLE XIII.-Boll-weevil mortality in 150!.

\begin{tabular}{|c|c|c|c|c|c|c|}
\hline \multirow{2}{*}{ Class of forms. } & \multirow{2}{*}{$\begin{array}{l}\text { Number of } \\
\text { weevil } \\
\text { stages. }\end{array}$} & \multirow{2}{*}{$\begin{array}{l}\text { Percentage } \\
\text { of stages } \\
\text { alive. }\end{array}$} & \multicolumn{4}{|c|}{ Percentage of stages killed by- } \\
\hline & & & Climate. & Predators. & Parusites. & $\begin{array}{l}\text { All agen- } \\
\text { cies. }\end{array}$ \\
\hline 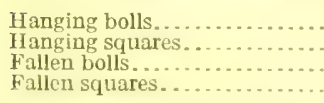 & $\begin{array}{r}1,534 \\
1,959 \\
573 \\
7,587\end{array}$ & $\begin{array}{l}53.33 \\
61.16 \\
54.82 \\
58.79\end{array}$ & $\begin{array}{l}37.94 \\
12.96 \\
27.74 \\
26.58\end{array}$ & $\begin{array}{r}5.21 \\
6.38 \\
15.00 \\
12.39\end{array}$ & $\begin{array}{r}3.52 \\
19.49 \\
2.44 \\
2.24\end{array}$ & $\begin{array}{l}46.67 \\
35.84 \\
45.18 \\
41.21\end{array}$ \\
\hline Totals and averages.... & 11,653 & 58.27 & 25. 84 & 10.56 & 5. 32 & 41. 73 \\
\hline
\end{tabular}


Following the plan adopted for the three preceding years these figures may be weighted for comparison with the earlier records:

TABLe XIV. - The hypothetical or werghted average mortaluty of the boll weevil in 1505.1

\begin{tabular}{|c|c|c|c|c|c|c|c|c|c|c|c|c|c|}
\hline \multirow[b]{3}{*}{ Class of ferms. } & \multirow{3}{*}{ 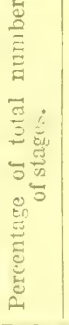 } & \multirow[b]{3}{*}{ 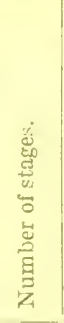 } & \multicolumn{11}{|c|}{ 1909-Mortality from- } \\
\hline & & & \multicolumn{2}{|c|}{$\begin{array}{l}\text { Prolifer- } \\
\text { ation. }\end{array}$} & \multirow[b]{2}{*}{ 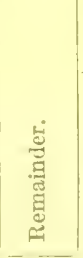 } & \multicolumn{2}{|c|}{ Climate. } & \multicolumn{2}{|c|}{ Predators. } & \multicolumn{2}{|c|}{ Parasites. } & \multicolumn{2}{|c|}{ Total. } \\
\hline & & & 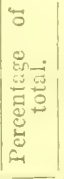 & 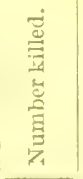 & & 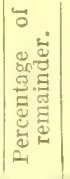 & 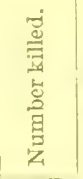 & 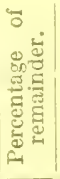 & 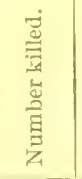 & 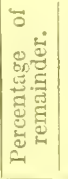 & 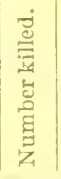 & 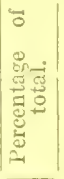 & 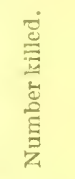 \\
\hline $\begin{array}{l}\text { Hanging bolls ......... } \\
\text { Hanging squares..... }\end{array}$ & $\begin{array}{l}0.75 \\
4.25\end{array}$ & $\begin{array}{r}73 \\
425 \\
\end{array}$ & $\begin{array}{r}6.30 \\
13.50\end{array}$ & $\begin{array}{r}4.7 \\
57.4\end{array}$ & $\begin{array}{r}70.3 \\
367.6 \\
\end{array}$ & $\begin{array}{l}37.94 \\
12.96\end{array}$ & $\begin{array}{l}2 u .7 \\
47.6\end{array}$ & $\begin{array}{l}5.21 \\
6.38\end{array}$ & $\begin{array}{r}3.7 \\
23.4\end{array}$ & $\begin{array}{r}3.52 \\
19.49\end{array} \mid$ & $\begin{array}{r}2.5 \\
71.6\end{array}$ & $\begin{array}{l}50.13 \\
17.29\end{array}$ & $\begin{array}{r}37.6 \\
200.0\end{array}$ \\
\hline Total hanging. . & 5. 00 & 500 & $|\ldots \ldots|$ & 62,1 & 437.9 & $|-\ldots|$. & 74.3 & $\ldots$ & 27.1 & $\ldots$. & 71.1 & $|\ldots| \mid$ & 237.6 \\
\hline $\begin{array}{l}\text { Fallen bolls........... } \\
\text { Fallen squares....... }\end{array}$ & $\begin{array}{l}14.25 \\
80.75\end{array}$ & $\begin{array}{l}1,425 \\
\mathrm{~s}, 075\end{array}$ & $\begin{array}{r}6.30 \\
13.50\end{array} \mid$ & $\begin{array}{r}90.0 \\
1,090.1\end{array}$ & $\begin{array}{l}1,335.0 \\
6,981.9\end{array}$ & 927.74 & $\begin{array}{r}370.3 \\
1,856.6\end{array}$ & $\begin{array}{l}15.00 \\
12.39\end{array}$ & $\begin{array}{l}200.2 \\
865.4\end{array}$ & $\left|\begin{array}{l}2.44 \\
2.24\end{array}\right|$ & $\begin{array}{r}32.6 \\
156.5\end{array}$ & $\begin{array}{l}48.63 \\
49.14\end{array}$ & $\begin{array}{r}693.1 \\
3,968.6\end{array}$ \\
\hline 'Total fallen.... & 95.001 & 9,500 & $|\ldots \ldots|$ & $|1,180.1|$ & $8,319.9$ & $\mid \ldots \ldots$ & $2,226.9$ & $|\ldots \ldots|$ & $1,065.6$ & $|\ldots \ldots|$ & $|189.1|$ & $|\ldots|$ & $4,661.7$ \\
\hline $\begin{array}{l}\text { Totals ancl aver- } \\
\text { ages.......... }\end{array}$ & 100.00 & 10,000 & 12.42 & $1,242.2$ & & 23.01 & $2,301.2$ & $10+2$ & 1,092. 7 & 2.63 & 203.2 & 18.99 & $4,899.3$ \\
\hline
\end{tabular}

This table shows a weighted decrease of 3.71 per cent for parasites and a weighted increase of 0.62 per cent for all agencies due to an increase in climatic control.

In the following table is given a comparison of the weighted average control by all agencies for the four years.

TABLE XV.-Weighted average mortality of the boll weevil, 1506-1509.

\begin{tabular}{|c|c|c|c|c|c|}
\hline \multirow{2}{*}{ Years. } & \multicolumn{5}{|c|}{ Weighted average mortality due to- } \\
\hline & $\begin{array}{l}\text { Prolifera- } \\
\text { tion. }\end{array}$ & Climate. & Predation. & Parasites. & $\begin{array}{l}\text { All agen- } \\
\text { cies. }\end{array}$ \\
\hline $\begin{array}{l}1906 \ldots \ldots \ldots \\
1907 \\
1908 \ldots \ldots \ldots \\
1909 \ldots \ldots \ldots\end{array}$ & $\begin{array}{l}12.42 \\
12.42 \\
12.42 \\
12.42\end{array}$ & $\begin{array}{l}24.39 \\
28.16 \\
17.83 \\
23.01\end{array}$ & $\begin{array}{l}24.85 \\
16.18 \\
11.77 \\
10.92\end{array}$ & $\begin{array}{l}2.94 \\
3.83 \\
6.34 \\
2.63\end{array}$ & $\begin{array}{l}64.61 \\
60.61 \\
48.37 \\
48.99\end{array}$ \\
\hline A verage...... & 12.42 & 24.45 & 15.93 & 3.93 & 56.73 \\
\hline
\end{tabular}

In view of the fact that certain cotton varieties retain the infested squares more than others, it is interesting to make another hypothesis on the basis that 50 per cent of the infested forms are hanging. The year 1908 is chosen to illustrate this phase of the subject. 
TABLE XVI.-A hypothetical average mortality of the boll weevil in square-retaining varieties. ${ }^{1}$

\begin{tabular}{|c|c|c|c|c|c|c|c|c|c|c|c|c|c|}
\hline \multirow{3}{*}{ Class of forms. } & \multirow{3}{*}{ 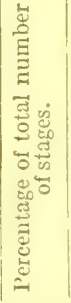 } & \multirow[b]{3}{*}{ 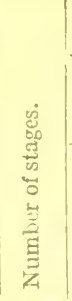 } & \multicolumn{11}{|c|}{ 1908. Mortality from- } \\
\hline & & & \multicolumn{2}{|c|}{$\begin{array}{c}\text { Prolifera- } \\
\text { tion. }\end{array}$} & \multirow[b]{2}{*}{ 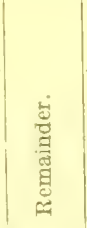 } & \multicolumn{2}{|c|}{ Climate. } & \multicolumn{2}{|c|}{$\begin{array}{l}\text { Preda- } \\
\text { tors. }\end{array}$} & \multicolumn{2}{|c|}{ Parasites. } & \multicolumn{2}{|c|}{ Total. } \\
\hline & & & 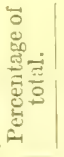 & 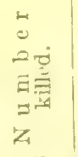 & & 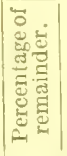 & 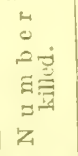 & 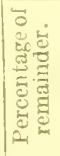 & 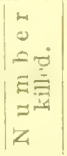 & 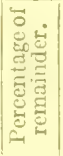 & 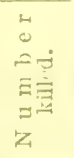 & 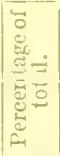 & $\begin{array}{l}4 \\
0 \\
=- \\
E \bar{\Xi} \\
=\bar{\Xi} \\
z\end{array}$ \\
\hline $\begin{array}{l}\text { Hanging bolls........ } \\
\text { Hanging squares.... }\end{array}$ & $\begin{array}{r}7.50 \\
42.50\end{array}$ & $\begin{array}{r}750 \\
4,250\end{array}$ & $\begin{array}{r}6.3 \\
13.5\end{array}$ & $\begin{array}{r}47.2 \\
573.7\end{array}$ & $\left|\begin{array}{r}702.8 \\
3,676.3\end{array}\right|$ & $\left|\begin{array}{l}38.48 \\
19.24\end{array}\right|$ & $\begin{array}{l}270.4 \\
707.3\end{array}$ & $\begin{array}{l}3.97 \\
9.50\end{array}$ & $\left|\begin{array}{r}27.9 \\
360.3\end{array}\right|$ & $\left|\begin{array}{r}8.15 \\
21.70\end{array}\right|$ & $\begin{array}{r}57.3 \\
797.8\end{array}$ & $\begin{array}{l}53.7 \\
57.3\end{array}$ & $\begin{array}{r}402.8 \\
2,439.1\end{array}$ \\
\hline Total hanging.-. & 50.00 & 5,000 & $|\ldots|$. & 620.9 & $4,379.1$ & $\ldots$ & 977.7 & $\ldots$ & 358.2 & $\ldots$ & 855.1 & $\ldots$ & $2,841.9$ \\
\hline $\begin{array}{l}\text { Fallen bolls .......... } \\
\text { Fallen squares. . . . . }\end{array}$ & $\begin{array}{r}7.50 \\
42.50\end{array}$ & $\left|\begin{array}{r}750 \\
4,250\end{array}\right|$ & $\left|\begin{array}{r}6.3 \\
13.5\end{array}\right|$ & $\begin{array}{r}47.2 \\
573.7\end{array}$ & $\mid \begin{array}{r}702.8 \\
3,676.3\end{array}$ & $\left|\begin{array}{l}20.08 \\
20.30\end{array}\right|$ & $\begin{array}{l}141.1 \\
746.3\end{array}$ & $\begin{array}{r}5.95 \\
15.18\end{array}$ & $\left|\begin{array}{r}41.8 \\
558.1\end{array}\right|$ & $\left|\begin{array}{l}3.71 \\
7.15\end{array}\right|$ & $\begin{array}{r}20.1 \\
202.8\end{array}$ & $\begin{array}{l}34.1 \\
50.3\end{array}$ & $\begin{array}{r}256.2 \\
2,140.9\end{array}$ \\
\hline Total fallen..... & 50.00 & 5,000 & & 620.9 & $4,379.1$ & & 887.4 & ...... & $\mid 599.9$ & $\mid \ldots$. & 288.9 & $\ldots$ & $2,397.1$ \\
\hline $\begin{array}{l}\text { Totals and arer. } \\
\text { ages.......... }\end{array}$ & 100.00 & 10,000 & & $1,241.8$ & & 18.65 & $1,865.1$ & 9.88 & 958.1 & 11.44 & $1,144.0$ & 52.39 & $5,239.0$ \\
\hline
\end{tabular}

1 Given 10,000 weevil stages.

This series of tables, wherein the mortality of the weeril is given an accurate basis for comparison, brines to light some very important points. This is especially the case in Table XVI, which is based upon the hypothesis that 50 per cent of the infested forms are hanging. By comparing this hypothesis for the year 1908 with the table of the same year in which it is considered that only 5 per cent of the forms are hanging, it will be noticed that under the condition of the greatest proportion of hancring squares the total control of the weevil would be 52.39 per cent and the number of parasites to 101000 weevil stages would be 1,154: whereas, with the smaller proportion of hanging foums, the total control of the weevil would he fis.:37 per cent and the total number of parasites $63 \pm$ to 10,000 wereil stages. Now this shows a gain of 4 per cent in the actual control of the weevil and almost double the number of parasites to 10.000 weeril stages. Naturally, under such conditions it would follow that the parasitic control would be even hicher than that which has heen used as a basis for the estimate and would increase in rapid proportion. In view of this showing of the farct that the larger the moportion of hanging squares to the entire amount of infested forms, the larger the insect control becomes, we recommend that those who are interested in the breeding of cotton varieties attempt to secure rarieties of cotton which will combine the necessary qualitics of productiva ness, length of lint, and early maturing with the square-retainime tendency. It may be pointed out that the varieties hnown as Rubiec and Cook's Improved are not only conspicuous for the square-retaining qualities but also for their desirability under boll-weevil concli- 
tions. Several other varieties have been noticed to have this same tendency, but they have not the other characteristics to recommend them. In this connection we refer the reader to section 4 (p. 21), in which it has been shown that at least two States have had a higher arerage control of the boll weevil in hanging squares than in fallen squares when all of the records available are considered. It will also be noticed in section 5, under Table XI, giving the actual conten of the boll weeril in 1908, that hanging squares and hanging bolls were decidedly in the lead in the total control over either fallen squares or fallen bolls. While this has not been the case in the other years under consideration, we nevertheless consider that the presence of a nursery for the parasites in the field is most desirable. Undoubtedly these hanging squares constitute such a nursery.

\section{A STUDY OF HOW AGRICULTURE MODIFIES INSECT CONTROL.}

From studies made during 1907 the following comparisons may be made to show the number of factors that it is actually necessary to consider in order that differences in parasitism may be understood.

At Arlington, Tex., records were kept on a field in the red loam post-oak country or "cross timbers," another in the Trinity River bottoms, and a third on the black waxy prairie. The first was planted March 12, the second April 1, the third April 5. On August 28 the weevil infestation of squares in the timbers was 80.5 per cent, in the bottoms 94.3 per cent, and on the prairie 21.4 per cent. At the same time the parasitism in falien squares on the timbers was 3.12 per cent, in the bottoms 1.9 per cent, and on the prairie 2.56 per cent. In the timbers the parasitism of hanging squares was 39 per cent and in the bottoms 24.78 per cent. The variable factors are soil, flora, time of planting, variety of cotton, and weevil abundance. Hanging squares were found in 1906 to be more highly parasitized in timher lambl than on the prairie, and fallen squares inversely. There appears to be an indication of the value of early planting. This first field was the eirliest field known in the vicinity and it showed a high parasitism in hanging forms throughout the season.

At Calvert, Tex., were two fields on the prairie, one planted March 11 and 12, the other April 1. On June 21 the weevil infestation of the first was 18 per cent and of the second 21 per cent. On July 5 the parasitism in the first was 2 per cent and in the second nothing.

At Denison, Tex., were two fields, one in the red clay, the other on sandy loam, neither surrounded by timber. On the first the stalks were burned February 28, on the second March 15. Both were planted March 30. On August 27 the weevil infestation on the first was 88.3 per cent, on the second 87.6 per cent; the parasitism in fallen squares on the first was 6.31 per cent, on the second 2.85 per 
cent; the parasitism in hanging squares on the first was 5.79 per cent, on the second 11.53 per cent. Here the only rariable conditions were soil, possibly weeds, and time of plant destruction. The parasitism in the two classes of forms was diametrically reversed.

At Terrell, Tex., were two fields on the sandy prairie, both planted in March, but having different weeds present. The weevil infestation August 26 on one was 65.2 per cent, on the other 97.5 per cent, while the parasitism in hanging squares on the first was 29.5 per' ('ent and on the second 25.6 per cent. The variables were field surroundings and weevil abundance.

The unknown influence which entered most of these examples was very probably the relative abundance of the difierent species of parasites. This may best be illustrated by the hanging squares from the timbers and bottoms at Arlington, which are quoted above. In the timbers the determinable para-iten proved to he 16 Eurytoma tylodermatis, 10 Microbracon mellitor, 6 Cerambycobius cyaniceps, 5 Hicrodontomerus anthonomi, and 3 Catolaceus spp. In the bottoms there were 17 Cerambycobius cyaniceps, 13 .Therodontomerus anthonomi, 10 Eurytoma tylodermatis, S Catolaccus spp., and 7 Microbracon mellitor. The rank of the species was almost entirely reversed.

Probably the most important point in the entire set of examples is that the earliest crop had the most parasites. To show this in another way we may refer to the conditions on the experimental farm at Dallas. The first part of the field to put on squares was the first part to show parasites. On July 8 infested squares were to be found in six plats, but only on this earliest plat was there any parasitism5.7 per cent. On July 19 it and the adjacent plat were still considerably in the lead.

That the earliest field should show the highest parasitism was expected by the writers in riew of the early spring observations. The parasites in hibernation, whether on the boll weevil or on winter cohosts, all reached maturity in the latter half of Mfarch at Dallas. It was reasoned that cotton, squaring and attacked by April 15, would get the hibernated parasites in any part of the State; that cotton squaring and attacked by May 1.5 would get the first generation of parasites from the cohosts, and so on. It is reasonable to expect that cotton with squares infested in season to attract hibernated parasites or a new brood from cohosts will fare better than cotton that commences squaring when all the parasites are concentrated upon neighboring cohosts. This cotton must wait until the period of the farored cohosts begins to wane before the parasites will begin to seek new scenes of activity. Although it was so reasoned, it was hardly expected that there would be sufficient proof to warrant. voicing the proposition. 
A series of examinations was made in the vicinity of Victoria, Tex., in 1907 and 1908. On October 9, 1907, Mr. Cushman noted that fall destruction of the cotton was being carried on quite extensively, but in different manners. On the east side of the river, south and east of town, was an area in which practically all of the cotton had been defoliated by the cotton leaf-worm. This area was separated by the river and by a wide strip of huisache timber from other cotton areas. In other directions were located fields stripped by grazing, some that were plowed under, and one field only was found which had received no treatment.

On June 17, 18, and 19, 1908, fallen squares from several of these fields were examined, with the following results:

TABLE XVII.-Boll-wecvil mortality in various cotton fields, Victoria, Tex., 1908.

\begin{tabular}{|c|c|c|c|c|c|}
\hline \multirow{2}{*}{ Treatment, 1907.} & \multirow{2}{*}{$\begin{array}{c}\text { Total } \\
\text { stages. }\end{array}$} & \multirow{2}{*}{ Total. } & \multicolumn{3}{|c|}{ Percentage of mortality, 1908.} \\
\hline & & & Climate. & $\begin{array}{l}\text { Preda- } \\
\text { tors. }\end{array}$ & Parasites. \\
\hline 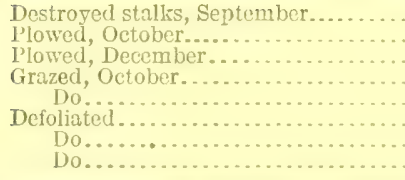 & $\begin{array}{l}314 \\
296 \\
354 \\
144 \\
290 \\
480 \\
513 \\
375\end{array}$ & $\begin{array}{l}18.18 \\
13.80 \\
60.70 \\
44.40 \\
37.50 \\
293.30 \\
52.80 \\
23.70\end{array}$ & $\begin{array}{r}5.43 \\
7.70 \\
14.40 \\
24.30 \\
25.50 \\
20.60 \\
27.00 \\
16.50\end{array}$ & $\begin{array}{r}4.14 \\
3.00 \\
41.20 \\
15.97 \\
7.20 \\
2.50 \\
11.10 \\
3.10\end{array}$ & $\begin{array}{r}9.23 \\
3.00 \\
5.08 \\
4.16 \\
4.80 \\
6.20 \\
14.40 \\
4.50\end{array}$ \\
\hline
\end{tabular}

These striking differences in the percentage of control can not be attributed to the differences of treatment in 1907, although that may have had a bearing. The diflerent fields had different weeds and phints surrounding them, they received diflerent treatment in the spring of 1908, and there are many other reasons why no one basis of comprarion can be chosen. The table is offered to illustrate low wide a diference in natural control can be found in fields only a few miles aprat and proves conchusely the ralue of individual effort in the fight against the weevil.

Sumerous ofher instances aro contained in the notes that are quite as striking as the one to which reference has been made. There is every reason why each planter should follow out as complete a program against the weevil as he can, because each effort reduces the total infestation of his neighborhood.

\section{CLIMATIC CONSIDERATIONS.}

The elimate of the hibernating season of $1906-7$ was very unusual, so much so that the boll weevil hardly became quiescent, and the emergence was largely during March, whereas normally it is in April. The boll-weevil parasites mature simultaneously with the 
great wave of boll-weevil emergence. A glance at the aceompanyine diagrams (firs. 4, 5) will show that in Louisiana tho monthly mean temperafure was from $3^{\circ}$ Fahrenheit (November) to $10^{\circ}$ (January) higher than the normal, and in Texas it varied from normal (November) (a $10^{\circ}$ above normal (Nareh) during the entire winter. On the other hand, the accumulated moisture from Norember 1 , 1906 , fo March 1, 1907, in Lonisiana was 5 inches helow mormal and in Texas 1 inch below normal.

Cotton was planted in Mareh and April (1907) and normally would have squared in May and June, but it was retarded a montle by the low temperature in April and May, during which months the monthly mean temperature was $2^{\circ}$ to $3^{\circ}$ below normal in Louisiana and $3^{\circ}$ to $6^{\circ}$ below normal in Texas. In addition to the cold of the spring, the precipitation in Lurisiana from Mareh 1 to July 1 was 7 inches above the normal and in Texas 2 inches above. This cold and the presence of volunteer eotton tided the boll weevi! over unt il the planted cotton was up. The parasites were obliged to seck cohosts from March 15 until late in May or in ofune. The cold, damp weather undoubtedly retarded their development so that the first generation was ready to attack such boll weevils as were hreeding late in May and early in Junc. As only a few ficlds held this advantage to the parasites, these fields naturally berame mueh better stocked with parasites, as las been pointed out in another paragraph.

The summer and earty fall months showed a slight deficieney in rainfall and a sighty higher mean temperature-to such an extent, howerer, that the season was eomsidered dry, for the cotton did not put on a very luxuriant foliage, and ílus gave the sum plenty of play on the fallen squares. The result is evidenced by the high percentage of mortality from heat shown in the mortality tables. The increase in parasitism may be ascribed to the same cause.

The mean temperature of October, 1907, was normal in Texas, but $10^{\circ}$ above normal in Louisiana. This warm season was followed by a very sudden drop in temperature on November 11, the "norther" lasting until the isth. This caused the Norember mean in both States to he $3^{\circ}$ below normal (Texas $53^{\circ} \mathrm{F}$., Louisiana $56^{\circ} \mathrm{F}$.). In both States during this one month the precipitation was 3 inches above the normal. In northern Texas about 30 per ecnt of the adult weevils were killed by cold. 'The temperature at Dallas ${ }^{1}$ reacher $14^{\circ}$ on November 13 , which was $11^{\circ}$ colder than was experienced in 1906 and $21^{\circ}$ lower than at any time in November, 1905. The boll weevils were not prepared for this cold, as they were still in great

1 The record was made both by the minimum thermometer and the self-registering thermograph at the laboratory in East Dallas, and is a few degrees lower than the official record at Oah Cliff, about 5 miles to the west and across the 'Trinity River.

16844-Bull. $100-12-3$ 
numbers on the plants and many immature stages were developing in green squares and bolls.

Table XVIII gives the results of the examinations made immcdiately after the freeze.

Table XVIII.-Mortality of the boll weevil in Texas, November, $190 \%$.

\begin{tabular}{|c|c|c|c|c|c|c|c|c|c|}
\hline \multirow{2}{*}{ Place. } & \multirow{2}{*}{ Date. } & \multirow{2}{*}{ Form. } & \multirow{2}{*}{ Location. } & \multirow{2}{*}{$\begin{array}{l}\text { Condi- } \\
\text { tion. }\end{array}$} & \multirow{2}{*}{ Stages. } & \multirow{2}{*}{$\begin{array}{l}\text { Mortal- } \\
\text { ity. }\end{array}$} & \multicolumn{3}{|c|}{ Mortality duo to- } \\
\hline & & & & & & & Cold. & $\begin{array}{l}\text { Para- } \\
\text { siles. }\end{array}$ & $\begin{array}{l}\text { Other } \\
\text { causes. }\end{array}$ \\
\hline 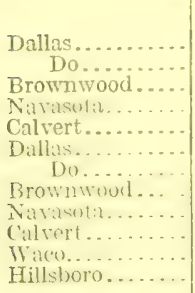 & 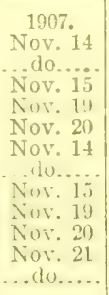 & 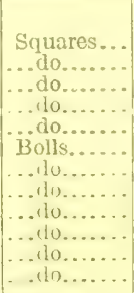 & 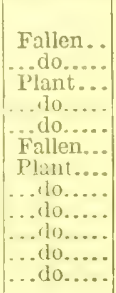 & 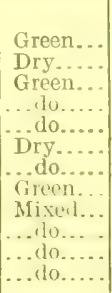 & $\begin{array}{r}93 \\
151 \\
2 \\
(1) \\
(1) \\
13 \\
8 \\
7 \\
56 \\
21 \\
16 \\
27\end{array}$ & $\begin{array}{r}\text { Per ct. } \\
97.7 \\
47.0 \\
100.0 \\
100.0 \\
100.0 \\
30.7 \\
37.5 \\
100.0 \\
96.3 \\
95.2 \\
100.0 \\
100.0\end{array}$ & $\begin{array}{r}\text { Per ct. } \\
96.7 \\
30.4 \\
100.0 \\
11 i 1.0 \\
100.0 \\
7.6 \\
100.0 \\
57.1 \\
38.0 \\
50.0 \\
63.0\end{array}$ & 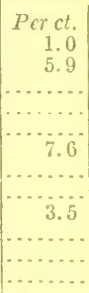 & 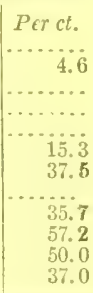 \\
\hline $\begin{array}{l}\text { Totals and } \\
\text { averages. }\end{array}$ & & $\left\{\begin{array}{l}\text { Squares... } \\
\text { Bolls..... }\end{array}\right.$ & & $\left|\begin{array}{ccc}\cdots & \ldots & \ldots\end{array}\right|$ & $\begin{array}{l}246 \\
148\end{array}$ & $\begin{array}{l}66.3 \\
88.5\end{array}$ & $\begin{array}{l}59.8 \\
49.3\end{array}$ & $\begin{array}{l}4.0 \\
2.0\end{array}$ & $\begin{array}{r}2.8 \\
237.1\end{array}$ \\
\hline $\begin{array}{l}\text { Totals and } \\
\text { averages. }\end{array}$ & & & 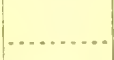 & & 394 & 74.3 & 55.3 & 3.2 & 15.8 \\
\hline
\end{tabular}

1 Several.

2 Most of the death from "other causes" in bolls was due to proliferation, which seems to be stimulated by frosts.

From this small number of stages no general statement cain be made. Of the 394 stages 55.3 per cent were killed by cold. Of the stages in green squares or bolls, 98 per cent were killed by the cold.

The most interesting point is that although 98 out of 100 weevil stages in ereen forms were killed, a paraite larva was foumd to have just hatched from its egg on a weevil larva killed by cold. Three other similar cases were found in dry forms. Seventeen cases of parasitism were found on the 394 stages. Among these were two liviner egrs of which one was an entirely new type and also two pupe which proved to be Habrocytus piercei.

The remainder of the winter of 1907-8 - that is, from December to March 1-had a mean temperature a few degrees above the normal, but with several severe cold spells. During the four winter months the precipitation in both States was above the normal. The short cold spells with warmer intervening weather and heavier rainfall were disastrous to the boll weevil. The February examination to ascertain the mortality of the weevil indicated about 98 per cent mortality. As a result of the extreme scarcity of weevils in the spring and summer in most parts of Texas, there was a great reduction in the number of parasites. In fact, in the northern portion of the Texas black prairie the parasites were forced to seek other hosts. A killing lreeze in tovember, 1908, again killed many boll weevils. 
Following the cold of November, 1908, the winter was unusually warm, being at least $5^{\circ} \mathrm{F}$. above the normal in both Louisiana and Texals. From March 15 to July 15, in both States, the temperature was almost normal. IIowever, by this time there was an acemulated deficiency of precipitation in each State of several inches. The months of July and August in Texas were extremely warm and many places recorded the maximum temperatures for their entire preriod of records. While the heat was less excessive in Louisiana, it nererthelesis reached very high points. This extreme weather during these two monthis had a tremendous eflect upon the boll weeril and upon its parasites, although records taken after some of the hot test days showed that the mortality of the boll weevil from the heat was considerably higher than the mortality of the parasites of the boll weevil. After the middle of August a pariod of renewal arowth ol' the cotton plant gave the boll weevil an opportunity for increased development and consequently permitted a large number of weevils to mature before the hibernation season. Incidentally with this fall brood of weevils, we find that there was a very great increase in the parasites, especially in Louisiana. The following two diacrams (figrs. 4, 5) illustrate the temperature of the years under consideration.

\section{HOW INSECT CONTROL FOLLOWS THE DISPERSION OF THE BOLL} WEEVIL.

From an economie standpoint it is rery important to know what kind of natural control of the boll weeril can be expeeted in newly invaded country. Since 1904 it has been noticed that maximum infestation is generally reached by Lugust 1, and that simultaneously an extensive dispersion of the boll weevil takes place. At this perioul the boll weevils fly to fields many miles beyond the parasite.: The climatic conditions during the dispersion period are such as will not serious ly interfere with prolific breeding of the weevils in the newly infested territory. The extent of the dispersion is limited only by the number of weevils flying and the amount of lood supply available. In the fall of 1909 the sparse production of cotton in southern Mississippi brought about a dispersion of 120 miles into new territory.

On knowledge of the inserts which attack the boll weevil shows that most of them are derived from the parasites of similar weevils that are native to the recion infested. Therefore, if parasites and predators are present in the invaded region, it is reasonable to expect that they will immerliately begin attacking the boll weevil. This assumption has been proven in many definite cases. At Minden, Ia s, in 1906, a parasite larva was found in a green square infesiod by the first generation. At Roxie, Miss., where the weeviis had been present only a few weeks in September, 1908, ant work and parasite work 
were easily found. Later in the season of 1908, an isolated infestation was found at lioadside, in Yazoo ('ounty, Miss., about 40 miles beyond

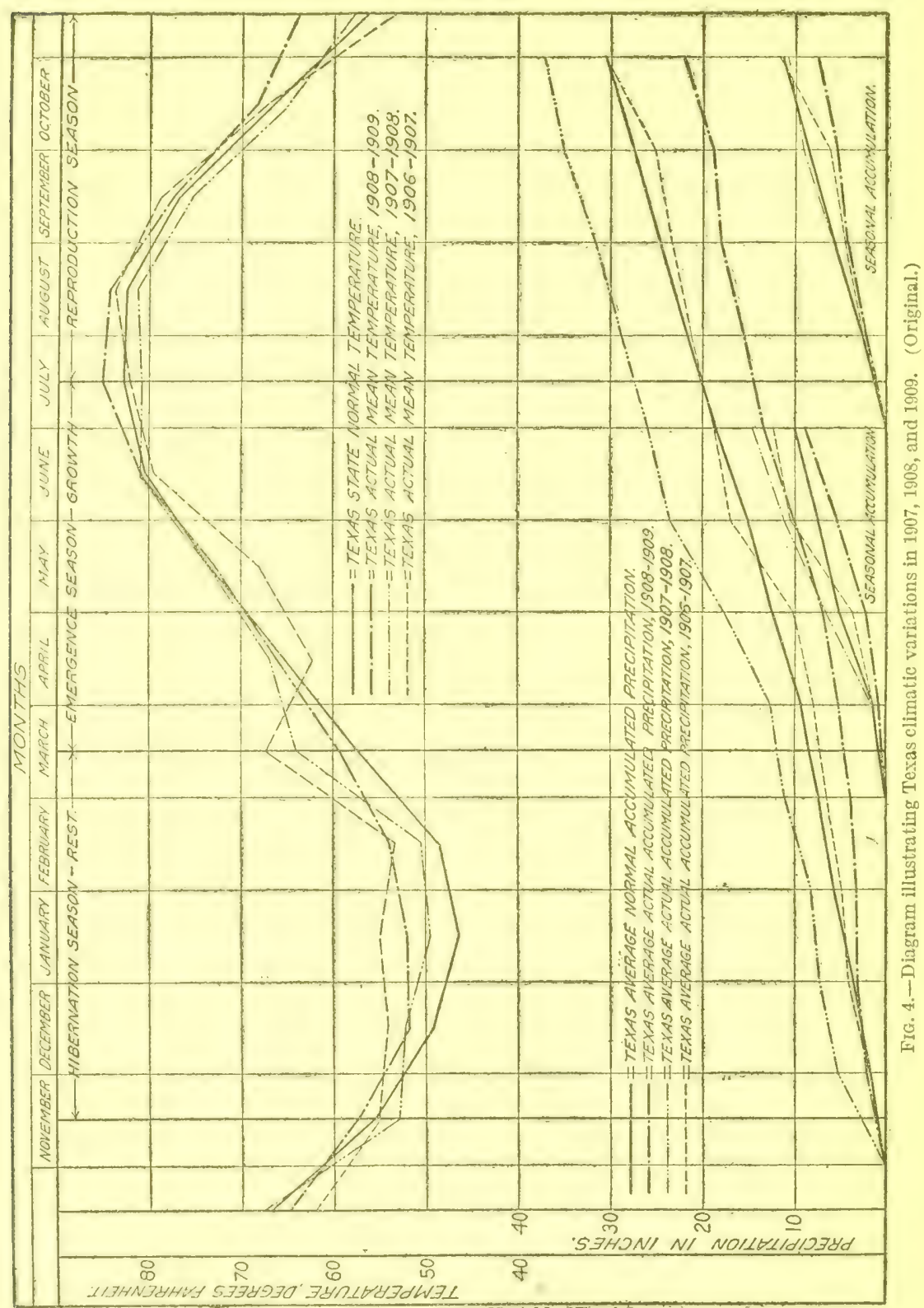

the regular line of infestation, but it was noticeable that the weevil was parasitized in this particular field. 
9. THE STATUS OF THE BOLL WEEVIL AND ITS CONTROL BY INSECTS.

1)uring the seasons of $190 \mathrm{~s}$ and 1909 the cxaminations of the boll weevil to determine its status demonstrated that there had been a

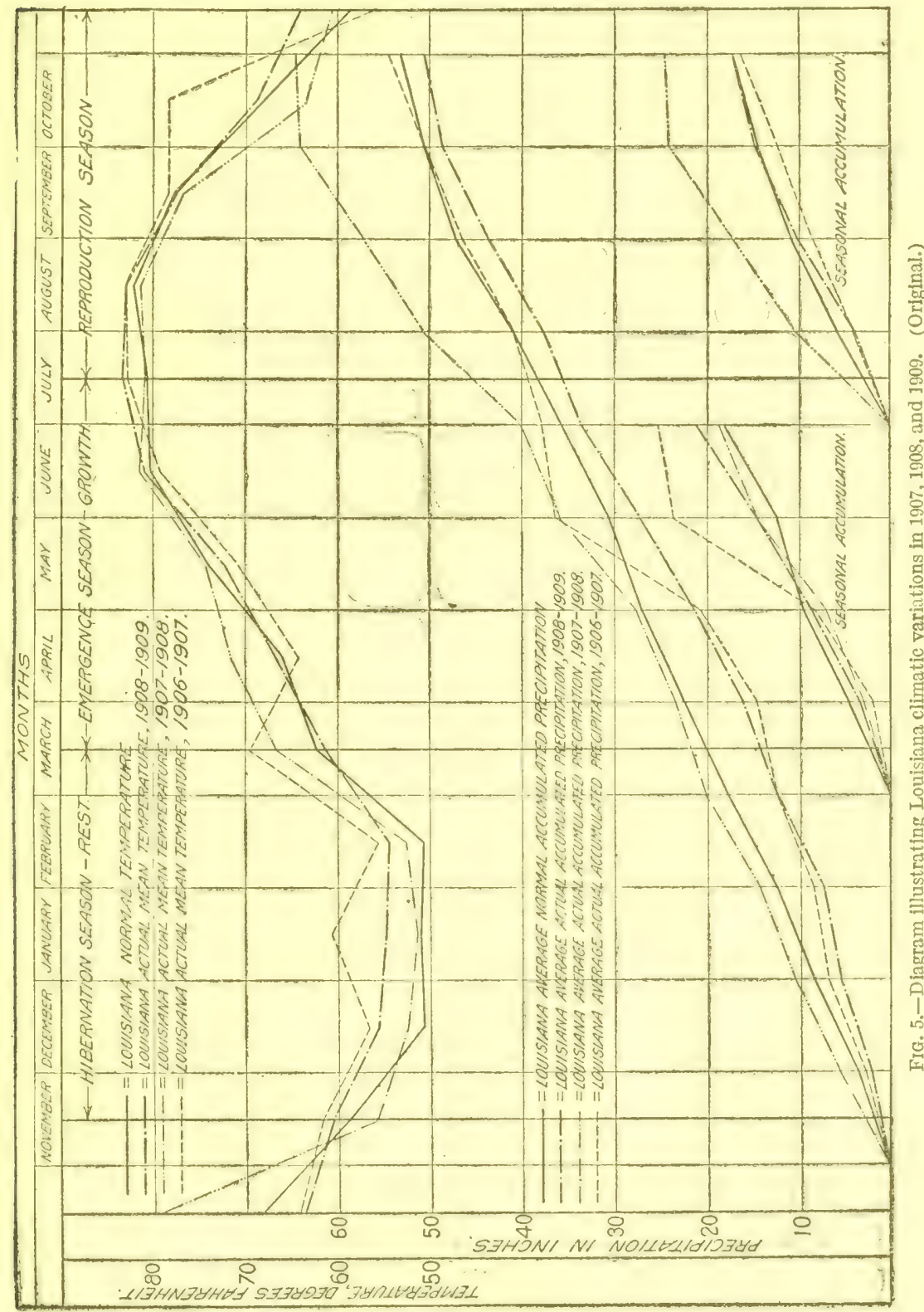

tremendous falling ofl of the weevil in all westem and northerm 'Texas. In Angust, 19)9, there was less than 10 per cent infestation in hall of 
Texas and in all of Oklahoma. At the same time a maximum infestation was found in all of that part of Louisiana lying south of the Red River and in Mississippi for about 2() miles east of Nat (hez. An analysis of the parasite records for this same season shows that the parasite control of the weevil in these sparsely infested regions of Texas was very light, whereas the control in the heavily infested regions of southern Louisiana and Mississippi was correspondingly very high. The inlerence drawn from this observation is either that the boll weevil had ceased to be the predominating weevil species for parasitic attack in the lightly infested region, or that the parasites hald been destroyed by the heat. That the parasites were not all destroyed by the heat is demonstrated by many records of the sime parasites on other species of weevils during the fall and winter of 1909 .

10. A BRIEF STATEMENT OF THE VARIOUS CLASSES OF CONTROL EXERCISED UPON THE BOLL WEEVIL.

Before passing from this part of the report, which deals with the general conditions obtaining, it is necossary to say a few words concerning the classes of control which are of importance in repressing the boll weevil. The first agency which is responsible for mortality of the weevils is the resistance of the cotton plant to attack, evidenced either by the toughness of the plant tissues which must be punctured, or by the proliferation of the tissues, which destroys the weevil eggs and larra by arushing. When the infested form l'alls to the ground or wither's on the plant it becomes immediately a subject for numerous other factors of control. Intense heat kills many stages. A large number of parasite species seek out infested squares for their prowony; myriads of ants, beotles, and mites find nourishing food by merely cutting their way into the infested forms and devouring the weevil stages. In addition to these, sudden cold freezes countless numbers of developing weovils. Neither are adults free from adrerse conditions. Many are killed by heat, or cold, or drowning; many are picked up by birds and lizards or preyed upon by other insects; and linally multitudes are starved on acrount of the ravages of other insects upon their food supply. In this report we are able to deal only with the three factors which are determinable in the control of immature weevils, namely, climate, parasites, and predators.

11. PRACTICAL CONGLUSIONS DERIVED FROM STATISTICAL STUDIES.

The following conclusions of economic importance have been reached from a study of this large series of statistics:

I. 'The month of August is the most important month for the control of the woevil by insect enemies. As this month is also the most 
important in the control affected by climate, it should be considered as one of the most critienl times of the rear for controlling the boll weevil. When a sudden drop in the temperature below freesing oceurs in the month of November before a large proportion of the weevils has entered hibernation, and while many are still immature, an excellent control of the species can be obtained. As, however, this is only an occasional occurrence, it can not be relied upon and every measure possible should have been carried out to prevent the weevils from going into hibernation at all.

II. II anging squares are the most important infested parts for the work of parasites, and fallen squares in a similar degree for the work of the predatory enemies. It has been demonstrated also that in certuin years the total control by all agencies is greater in hanging squares than in fallen squares, and furthermore that in the nore humid States this condition is the prevalent one.

III. It has been shown by examples that the total mortality of the weevil can bo increased in proportion as the number of hanging squares in a given area is increased and likewise that the proportion of parasites to weevils is increased. It is therefore recommended that plint breeders attempt to develop varieties of rotton which will retain the squares, but will also have the other desirable variotal characteristic's necessary for the production of an early cotton crop.

IV. The insect control of tho boll weevil is dependent in a large measure upon the operations of the farm and for this reason all those field practires which have been included in the srstem of enltural control of the boll weeril are further recommended as tending to increase the insect control.

\section{PART II. BIOLOGICAL COMPLEX.}

In Part I of this bulletin one set of facts, composed of statistice, was dealt with, and it was merely hinted that the causes of these conditions were very complex. In this part is presenterl another series of facts, even more significant than the first, but much more difficult to present in a tangible manner. The study of these biological factors received its first impetus when at Clarendon. Tex., in 190.). Mr. C. R. Jones and the senior author were fortunate cnough to leam the biologies of three species of weevils and to find that all these were parasitized more or less abundantly by the same parasites an is the boll weeril. It was already known that some of the parasites of the boll weevil attacked other weevils, but the significance of this fact had not been realized. 
With this simple begimning the sonch for other hosts of the bollweeril parasites was started and we have now built up the knowledge of the following complex:

Owing to the complicated nature of the data to be presented in this part, these have also been arranged in the following sections:

1. A list of the insect enemies of the boll weevil.

2. The hosts of boll-weevil parasites.

3. Mites which attack the boll weevil.

4. Flies which parasitize the boll weevil.

5. The hymenopterous parasites of the boll weevil.

6. Biological notes upon the parasites of the boll weevil.

7. The development of the parasites.

8. The distribution of the parasites.

9. The parasite seasons.

10. Adjustment to new hosts.

11. Beetles which prey upon the boll weevil.

12. Lepidopterous larvæ which are incidentally predatory upon the boll weevil.

13. Ants which prey upon the boll weevil.

14. Biology of the cohosts of the boll-weevil parasites.

15. A list of the host plants of the cohost weevils.

16. A summary of the most important biological facts.

\section{A LIST OF THE INSECT ENEMIES OF TIIE COTTON BOLL WEEVIL.}

The boll weevil is known to be attacked by 29 species of parasites, while 20 species of predators attack the immature stages and 6 species of predators attack the adults. These species are listed as follows:

\section{Arachnida.}

Acarina. Sarcoptoidea.

Tarsonemidæ. Pediculoidinæ.

Pediculoides ventricosus Newport (parasite on larva), Mexico.

Pediculoides sp. (parasite on larva), Louisiana, Texas.

Tyroglyphidx.

Tyroglyphus breviceps Banks (parasite on larva), Texas. Insecta.

Orthoptera. Mantoidea.

Mantide.

Stagmomantis limbatn IIahn (predator on adult), Texas.

Hemiptera-Heteroptera.

Reduviidæ.

A piomerus spissipes Say (predator on adult), Texas.

Coleoptera. Adephaga.

Carabidœ.

Evarthrus sodalis Le Conte (predator on adult), Louisiana, Texas.

Evarthrus sp. (predator on adult), Louisiana. 
Insecta-Continued.

Coleoptera. Polyphaga. Diversicornia.

Cantharidæ.

Chauliognathus spp. (predators on larva), Louisiana, Mississippi.

Cleridæ.

Hydnocera pallipennis Say (predator on larva), Texas.

Hydnocera pubescens Le Conte (predator on larva), Texas.

Cucujidæ.

Cathartus cassix Reiche (predator on larva), Texas.

Lepidoptera. Bombycoidea.

Noctuidæ.

Alabama argillacea Hübner (defoliator, cuts off food supply).

Hymenoptera. Formicoidea. ${ }^{1}$

Dorylidæ.

Eciton (Acamatus) commutatum Emery (predator on larva), Texas.

Poneridx.

Ectatomma tuberculatum Olivier (predator on adult) Guatemali.

Myrmicidx. Cremastogasterinæ.

Cremastogaster lineolata (Say) var. clara Mayr (predator on larva) Texas.

Myrmicidæ. Solenopsidinæ.

Solenopsis geminata (Fabricius) var. diabola Wheeler (predator on larva), Louisiana, Mississippi, Texas.

Solenopsis molesta Say (=debilis Mayr) (predator on larva), Oklahoma.

Solenopsis texana Emery (predator on larva), Louisiana, 'Texas.

Myrmicidæ. Myrmicinæ.

Monomorium minimum Buckley (predator on larva), Louisiana, Mississippi, Texas.

Monomorium pharaonis Linnæus (predator on larva), Arkansas, Louisiana, Oklahoma, Texas.

Pheidole sp, near flavens (predator on larva), Texas.

Pheidole crassicornis Emery (predator on larva), Texas.

Dolichoderidre.

Forelius maccooli Forel (predator on larva), Texas.

Dorymyrmex pyramicus Roger (predator), Cuba.

Dorymyrmex pyramicus (Roger) var. flavus McCook (predator on larva), Texas. Iridomyrmex analis André (predator on larva), Texas.

Formicidx.

Formica fusca subpolita perpilosa Wheeler (predator on adult), Mexico.

Formica pallidi-fulva Latreille (predator on larva), Arkansas.

Prenolepis imparis Say (predator on larva), Arkansas.

Hymenoptera. Chalcidoidea.

Chalcidide. Chalcidinæ. Smicrini.

Spilochalcis sp. (parasite), Texas.

Torymidæ. Monodontomerinæ.

Microdontomerus anthonomi Craw ford (parasite), Louisiana, Texas.

Eurytomidx.

Eurytoma tylodermatis Ashmead (parasite), Arkansas, Louisiana, Mexico, Oklahoma, Texas.

Bruchophagus herreræ Ashmead (parasite), Mexico.

Eurytoma sp. (parasite), Texas. 
Insecta-Continued.

Hymenoptera. Chalcidoidea-Continued.

Perilampidie.

Perilampus sp. ${ }^{1}$ (parasite), Louisiana.

Encyrtidx. Eupelminæ.

Cerambycobius cyaniceps Ashmead (parasite), Arkansas, Louisiana, Mississippi, Oklahoma, Texas.

Cerambycobius cushmani Crawford (parasite), Texas.

Cerambycobius sp. (parasite), Mississippi.

Pteromalidæ. Pteromalinæ.

Catolaccus incertus Ashmead (parasite), United States.

Catolaccus hunteri Crawford (parasite), Louisiana, Mississippi, Mexico, Texas.

Habrocytus piercei Crawford, Louisiana, Texas.

Lariophagus texanus Crawford (parasite), Texas.

Eulophidæ. Tetrastichinæ.

Tetrastichus hunteri Crawford (parasite), Louisiana, Mississippi, Texas.

Hymenoptera. Ichneumonoidea.

Ichneumonidæ. Pimplinæ. Pimplini.

Pimpla sp. (parasite), Texas.

Braconidæ. Sigalphinæ.

Sigalphus curculionis Fitch (parasite), Louisiana, Mississippi, Texas.

Urosigalphus anthonomi Crawford (parasite), Texas.

Urosigalphus schwarzi Crawiord (parasite), Guatemala.

Urosigalphus sp. (parasite), Texas.

Braconidæ. Braconinæ. Braconini.

Microbracon mellitor Say (parasite), Mexico, United States.

Braconidix.

Unknown species (parasite), Texas.

Diptera. Cyclorrhapha.

Phoridx.

Aphiochæta nigriceps Loew (parasite), Texas.

Aphiochxta fasciata Fallen (parasite), Texas.

A phiochæta pygmxa Zetterstedt (parasite), Texas.

Tachinide.

Myiophasia anea Wiedemann (determined by Coquillett) (parasite), Texas.

Ennyomma globosa Townsend (parasite), Louisiana, Texas.

Diptera.

HYPERPARASITES, ${ }^{2}$

I'lastophora (Pseudacteon) crawfordi Coquillett on Solenopsis geminata Fabricius.

\section{THE HOSTS OF BOLL-IVEEVIL PARASITES.}

As has just been stated, the boll weevil has 55 species of insects, which are known to attack it. Among the parasites are to be found 7 which are occasionally accidentally hyperparasitic. At least 1 parasite is known to attack one of the predators. The accidental predator (Alabrma argillacea) is attacked by 12 parasites, 46 predators,

\footnotetext{
1 This species may be a parasite of a Chrysopa larva or of some lepidopteron which had entered a weevil cell.

2 The enemies of Alabama argillacco. Hübner afford sone interesting sidelights ou the complexity of the biological relations of cotton insects.
} 
and 1 hyperparasite. Among these 46 predators are 6 which also prey upon the boll weevil. At least 1 very common predatory insect is known to prey upon many of the boll-meril predators. Fifty-five species of weerils are known to be attaleked as cohosts of 26 species of parasites and of the 19 species of preclator's which at ack the boll weevil. These 55 species of weevils are known to breed upon 91 species of plants, most of which are to be found in the vicinity of the cot ton ficlds. Three of these weerils somet imes breed upon the cotton plant. Among the great number of parasites which attack the 5i) cohost weevils, 44 species are definitely known to science and at least 6 species of hymenopterous parasites are known to attack these 44 species of parasites. This complexity could be carried still further, but probably enough has been stated to show how the many influences of nature are dependent upon one another. The statements are illustrated graphically in the aceompanying diagram (fig. (i).

The principal point of importance in all of these facts is that the boll weevil has been deriving its parasites from these 51 species of weevils and from other weevils which are not known to us, and there is every reason to believe that some of these other 44 species of parasites, or still additional ones to be discovered, may be drawn orer to the boll weevil as parasites in the future. The weevils serving as cohosts and the parasites are listed in the accompanying table (fig. 7) in such manner as to show the nature of the interrelationships.

It will be noticed from this table that 6 weevils, namely, Laria sallici, Laria exigua, Smicraulax tuberculatus, Anthonomus albopilosus, Tyloderma foveolatum, and Trichobaris texana each have 4 of the bull-weevil parasites; 4 weevils are attacked by 3 of the parasites, 15) of the weerils by 2 parasites each, and the remaining 37 by only 1 parasite each.

of the parasites, Cerambycobius cyaniceps attacks 18 hosts, Eurytoma tylodormatis attacks 16 hosts, ('atolaccus incertus 14, Catolaccus Thuntri 1:), and Ificrobrucon mellitor 12. These 5 parasites are also regarded as the most inportant parasites attacking the boll weevil itself. Perhaps this importance is due to the fact that they have a larger number of native hosts and are hence in greater abundance arotind the cotton fields than the parasites having fewer native hosts.

\section{MITES WHICH ATTACK THE BOL, WEEVIL.}

ACARINA. TARSONEMIDE.

The mites of the genus Peliculoiles are assuming an important rôle among insect parasites, two species being accredited to the boll weevil. 
Pediculoides ventricosus Newport (fig. 8). This mite has been somervhat prominent in the study of the boll weevil since its first notice in 1901 (Rangel, 1901) under the name of Pediculoides ven-

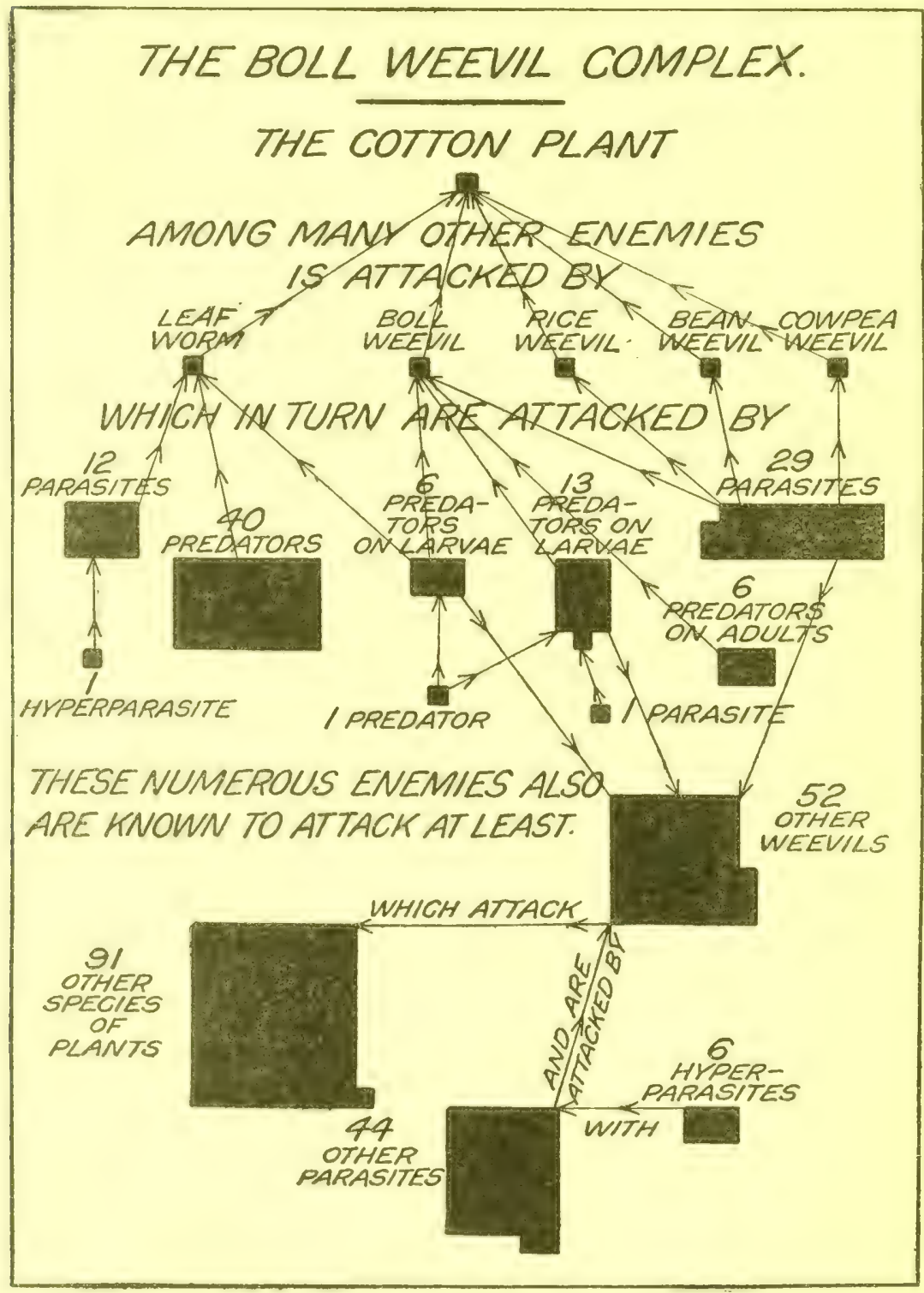

FiG, 6,-Diagram illustrating the boll-weevil complex. (Original.)

triculosus. Mr. Banks has stated that it may possibly be different from the European species, but as it is known throughout this country under the above name it is so quoted here. Mr. Rangel 


\section{THE OTHER HOSTS}

LARIA BISIGNATA

LARIA COMPRESSICORNIS

LARIA EXIGUA

LARIA OBTECTA

LARIA OCHRACEA

$\angle A R I A$ SALLAEI *

SPERMOPHAGUS ROBINIAE

BRACHYTARSUS ALTERNATUS

ARAECERUS FASCICULATUS

LIXUS MUSCULUS

LIXUS SCPOEICOLLIS

SMICRONYX TYCHOIDES

DESMORIS SCAPALIS

APION DECOLORATUM

APION GRISEUM

APION NIGRUM

APION ROSTRUM

APION SEGNIPES

MACRORHOPTUS SPMAERALCIAE TACHYPTERELLUS QUADRIGIBBUS SMICRAULAX TUEERCULATUS ANTHONOMUS AENEOLUS

ANTHONOMUS ALBOPILOSUS ANTHONOMUS APHANOSTEPHI ANTHONOMUS EUGENII ANTHONOMUS FULVUS ANTHONOMUS GRANDIS ANTHONOMUS HETEROTHECAE ANTHONOMUS LIGATUS ANTHONOMUS NEBULOSUS ANTHONOMUS NIGRINUS ANTHONOMUS SIGNATUS ANTHONOMUS SQUAMOSUS TVCHIUS SORDIOUS

CONOTRACHELUS AFFINIS

CONOTRACHELUS ELEGANS CONOTRACHELUS JUGLANDIS CONOTRACHELUS NASO CONOTRACHELUS NENUPHAR CHALCODERMUS AENEUS TYLODERMA FOVEOLATUM GERSTAECKERIA NOBILIS AULEUTES TENUIPES CRAPONIUS INAEQUALIS CEUTORHYNCHUS SP. RHINONCUS PYRRHOPUS BARIS CUNEIPENNIS BARIS SP

ORTHORIS CROTCHII

TRICHOBARIS COMPACTA TRICHOBARIS TEXANA TRICHOBARIS TRINOTATA AMPELOGLYPTER SESOSTRIS ZYGODARIS XANTHOXYLA BALANINUS NASICUS CALANDRA ORYZA TOTAL NUMEER OF HOSTS

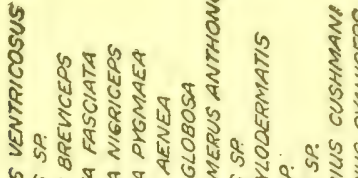

है है

岱

तas

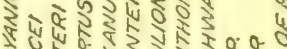

o

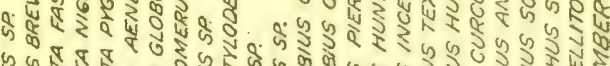

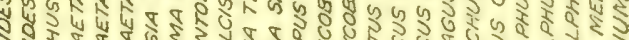

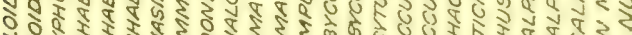
1 v

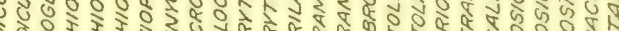
की Q

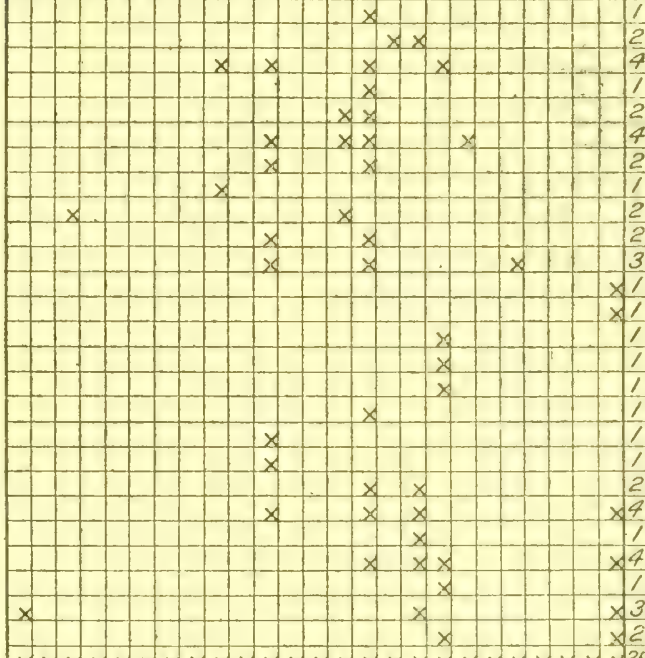
$\times x \times \times \times \times \times \times \times \times \times \times \times \times \times \times \times \times \times 2$

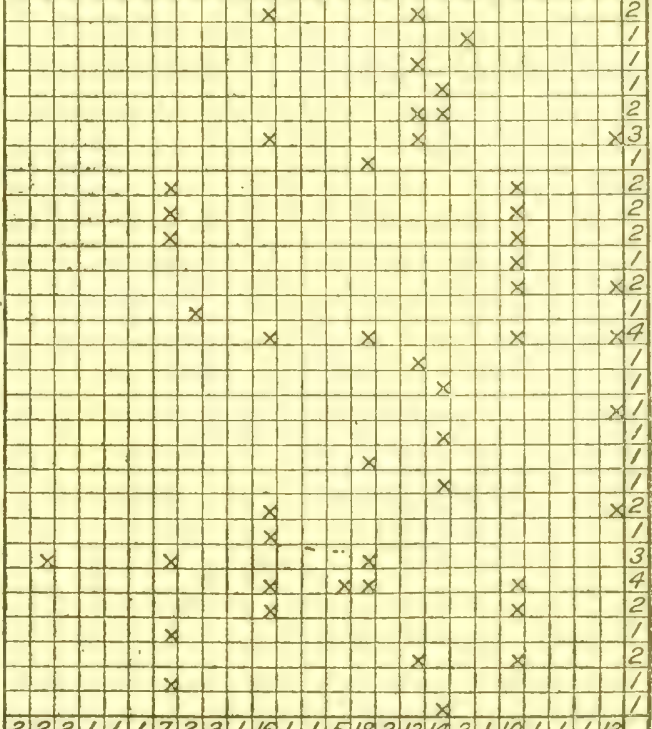
2द2///723//6//15/82/3/433/10////Va

Fig. 7.-Diagram giving the parasites of the boll weevil and their other hosts. (Original.) 
printel out that these mites reproduce viviparously and that their offipring are mature and fertile at birth. IIe found that when they attach themselves to a host the abdomen commences to inflate until it becomes many times larger than the thorax. The time required for engorgement varies from 22 to 5 days. Then the abdomen commences to grow, the young commence to leave the parent. The males fertilize the females before leaving the parent's body and shortly afterwalds die. An average of 100 female offspring to an individual was recorded. Rangel found that in 48 hours, 21 stages out of 40 in squares were attacked by the mites. In larger series of tests, after four days 50 out of 153 weevil stages in squares were attacked, or 32.6 per cent.

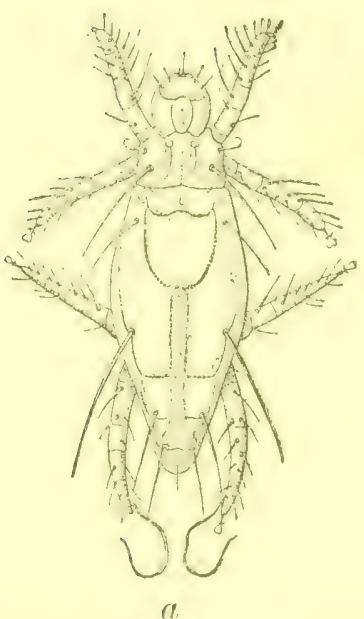

a.

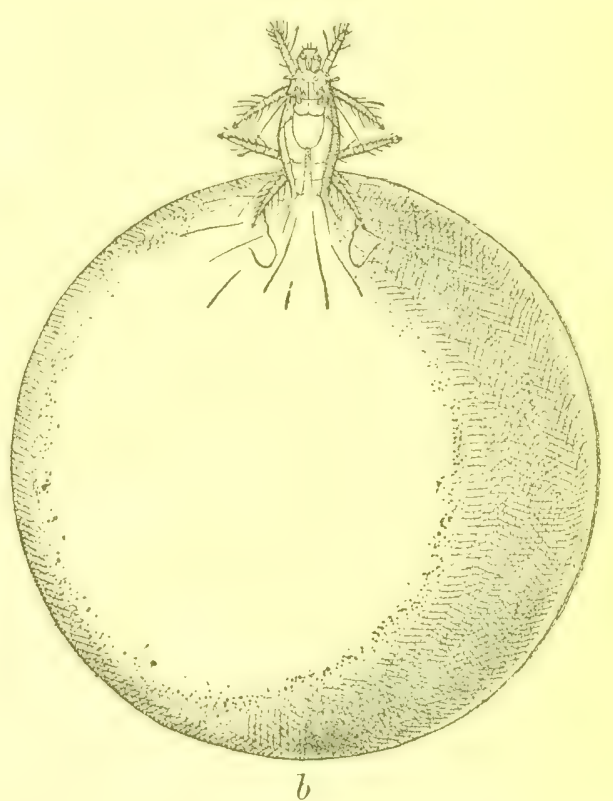

FIG. 8.-Pediculoides ventricosus: $a$, Adult female before inflation of abdomen with eggs and young; $b$, adult female after inflation of abdomen with eggs and young. Greatly enlarged. (Redrawn from Brucker.)

Pediculoides n. sp. This mite was discovered in the laboratory at Dallas, Tex., June 13, 1907, by the senior author. Careful observations on the length of generations were made with the following result:: $\Lambda$ gravid female was isolated June 13 and on June 17 there were 31 gravid mites. All but 5 were removed. On June 19 there were many oflepring, one mite being well grown. On June 21 the fourth generation began to appear. In other words, between June 13 and June 21, that is, in less than 8 days, there were two complete gencrit ions. Another genealogy was as follows: Parent isolated June 13, second generation began to appear Jume 14, were mature 
June 17, and reproducing June 19. On June 24 the third generation was reproducing. In this case there were 11 days covering two complete generations.

The mites appeared willing to feed on any insect foorl available, as they were first found feeding on stages of Trichobaris compacta, then on boll-weevil stages, and finally on a Baris, on boll-weevil parasites isolated in rearing tubes, and on IIydnocerd pubescens. They were reared readily on larve of Chtorion cyancum and Polistes rubiginosus. Mrr. John B. Railsback, of Forbing, Ia., found that they attacked the larva of the bollworm and other smooth caterpillars very readily.

\section{TYROGLYPHID E.}

Tyroglyphus breviceps Banks was described as a weevil enemy from Victoria, Tex. 'This, or a similar mite, was found to be very abundant at Calvert, 'Tex., in 1906.

\section{FLIES WHICH PARASITIZE THE BOLL WEEVIL.}

Very few Diptera are known to be primarily parasitic upon boll weevils, but the genera Myiophasia and Ennyomma in the Tachinidx seem to be confined to hosts of this nature. The genus Aphiochat a, of the Phoridre, contains at least 3 species which have been reared under circumstances pointing to primary parasitism. The larve of the tachinils work singly and those of Aphiocheta several to a host, but in both cases as endoparasites. When the former become full grown they completely fill the skins of the weevil larve and frequently the appendages of Myiophasia penetrate to the exterior. The weevil skin partakes of the character of parchment and hecomes a cocoon within which the fly larva pupates and from which the adult emerges. On the contrary the Aphiochæta larve leave the host when they have reduced it to a shell and pupate in the weevil cell.

The flies evidently prefer to attack weevil stages in moist, shaded spots in preference to sunny locations. By this habit they become very valuable in fields located in bottom lands where the dry conditions conducive to parasites liko the hymenopterous parasites are absent. The puparia of Myiophasia and Ennyomma are so near like that of the chalcidoid internal parasite Tetrastichus hunteri that they can be differentiated only by the larger size of the dipterous puparia.

PHORIDA.

Aphiochacta nigriceps Loew (determined by D. IV. Coquillett). Aphiochata fasciata Fallen (determined by D. W. Coquillett). Aphiochxta pygmæa Zetterstedt (determined by D. W. Coquillett). 
On September 12, 1906, in dry hanging bolls collected at Dallas, Tex., a werevil larva was fomml parasitized and isolated in a separate fube, with the following recorel: "Very small parasite larva on small weevil larva." On September 26 a single specimen of Aphiochxta nigriceps Loew was reared and the following note made by the senior author: "Found dipterous puparium, skin of hairy parasite larva (may be the dipteron or a hymenopteron); also remains of weevil larva." On October 6, 1906, in lianging bolls collected at Dallas a weevil larva was isolated with the note, "Weevil larva full of dipterous larvæ." Eleven larvæ left this host larva and pupated. On October 29, 7 Aphiochxta (?) fasciata Fallen and two A. pygmxa Zetterstedt were reared. At least the latter case seems to b very stome ovidence of primary parasitism. These flies are reared frequenty from holls and many are perhaps seavengers. 'Two other species, A. epeirx Brues and A. scalaris Loew, have also been reared from cotton forms at Calvert, Tex. Records made in 1911, at TalIulah, La., by Mr. Harry Pinkus, point conclusively to primary parasitism.

TACHINIDA.

Myiophasia ænea Wiedemann is recorded from a number of very important weevils. Of these, Balaninus nasicus Say (the acorn weevil), ('omolmalulus juglandis Le('onte (the walnut weevil), $1 \mathrm{~mm}$ Toglypter scsutris Tof'onte (the graperine gall-maker), Conotruchelus affinis Boheman (the hickory-nut weevil), and Conotrachelus elegans Say (tho pecan-gall weevil) are all weevils which enter the ground for pupation, carrying their parasites with them, and consequently it becomes necestary lor the flies to emerge from the weevil cell through several inches of earth before attaining freedom. The only weevils which this fy allacks and which do not enter the ground for pupation are the boll weevil and Trichobaris compacta Casey (the Jamestownweed pod weevil). Very few records have been made to ascertain its derelopmental period, but the three pecords at hand indicate from 22 to 29 days as the period from collection of the infested material to the mat urity of the fly. This period would cover largely the underground period only.

Ennyomma (Locwia) globosa Townsend. Several specimens of this fly were reared during 1907 by Mr. C. R. Jones at Alexandria, La., as primary parasites of the boll weevil. It is a very common parasite of Chalcodermus æneus Boheman (the cowpea-pod weevil) in the Southern States.

\section{THE HYMENOPTEROUS PARASITES OF THE BOLL WEEVIL.}

Sio mule information has been gained concerning the hymenopterous parasites of the boll weevil that it will be necessary to omit many of the technical facts learned about them. The present sec- 
fion is concerned with the sources of the parasites and with important records of their oncurrence, wh: le the other interesting facts to be presented are included in the five following sections:

CHAICIDOIDEA. CHALCIDIDE. CHALCIDINA. SMICRINI.

Spilochukis sp. A single male of this species was found dead in a weevil cell with the remains of the weevil and its own exuvium in a hanging square collected August 10, 1907, at Victoria, Tex.

TORYMIDA. MONODONTOMERINA.

Ificrodontomems anthonomi Crawford (fig. 9). Brachylarsus altermatus Say was formerly the only weevil recorded as a host of this species. $\Lambda$ male and female of this parasite were reared on Septem-

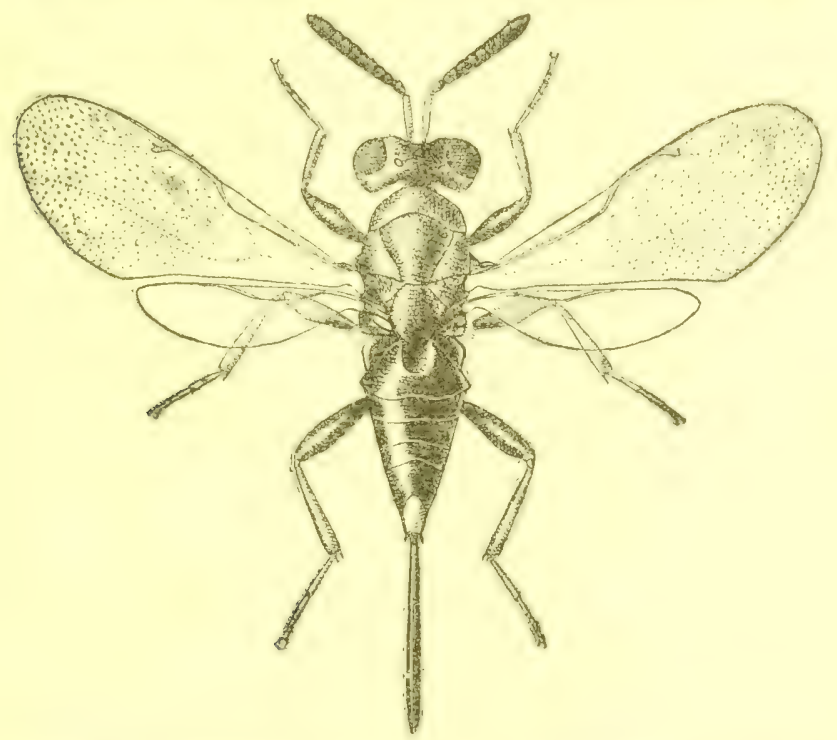

FIG.9.- Hicrodoniomerus anthonomi: Adult. Much enlarged. (Original.)

ber 12, 1967, from pods of the flowering shrub Amorptu frutiense at Dallas, Tex., which were highily infested by (Bruchus) Lurie cxigun IIorn. In 1906, in which year this parasite was first dicenvered, it ranked as seventh species in importance as a boll-weevil enemy. In 1907 it adranced to fourth place and was rery important in the central black-prairie region of Texas. In 1909 the easternmost limit of our records was Tallulah. La., which did not become inferted by the boll weevil until 1908 .

\section{EURYTOMIDE. EURYTOMINI}

Eurytoma tylodermatis Ashmead (Bruchophagus herrere A.hnead). This is without doubt one of the most important species under consideration, haring a range of distribution practically coexten$16844^{\circ}-$ Bull. $100-12-4$ 
sive with that of its host, the boll weevil. In previous publications it has been recorded as a parasite of Lixus musculus Say, L. scrobicollis Boheman, Apion segnipes Say, Anthonomus heterolhecx Pierce, Anthonomus squamosus LeConte, Tyloderma foncolatum Say, and Orthoris crotchii I.eConte. To this list may be added (Bruchus) Laria axigua Iforn in Amorpha pods at Dallas, Tex.; (Bruchus) Laria sallai Sharp in pods of huisache (Iachellia furnesiana) collected at Victoria, 'Tex.; Spermophagus robiniz Schönherr in pods of the loney and water locusts (Gleditsia triacanthos and (r. aquatica) in Louisiana; Mfacrorhoptus sphæralciæ Pierce in pods of Sphæralcia at Del Rio, Tex.; Smicraulax tuberculatus Pierce in mistletoe stems at Dallas, Tex.; Trichobaris texana LeConte in stems of Solanum rosiratum at (isco and Victoria, Tex.; and also Trichobaris trinotate Say; and finally it was reared in September, 1908, from Baris sp. in roots of ambrosia at Camden, Ark., by C. E. Hood.

Euryioma sp. A female primary parasite and a male accidentally secondary on Microbracon mellitor Say were reared from hanging squares collected August 10, 1907, at Victori̊, Tex.

PERILAMPIDA.

Perilampussp. A single individual was reared from the boll weevil in an isolated weevil cell by C. E. Hood from squares collected September 7, 1907, at Shreveport, La. Another specimen of Perilampus was reared from squares collected at Granbury, Tex., August 8, 1907, but its source could not be proved. If it were not for the definite record made by Mr. Ifood these species could hardly be placed in this list. This record may possibly be based upon the parasite of an intruder in the weevil cell instead of upon the weevil itself. It is not impossible that after several years this parasite may be found normally as a boll-weevil parasite, as was the case with Sigalphus curculionis.

ENCYRTIDE. EUPELMINA.

Cerambycobins cyaniceps Ashmead. The list of hosts of this species as previonsly recorded included Anthonomus albopilosus Dietz, (Bruchus) Laric obtecta Say, L. exigua Horn, Lixus musculus Say, Trichobaris texana LeConte, and Tyloderma foventatum Say. To this list may be added Laria bisignata. Iorn in pods of Acuan illinoensis; L. ochracea Schacfier in pods of Vicia sp.; L. sallæi Sharp in pods of huisache (Tachellia farnesiana); Spermophagus robinix Schönherr in pods of (ileditsia triacanthos at Alexandria, La.; Lixus serobicollis Boheman in stems of Ambrosia trifida and A. psitostachya; Apion rostrum Say in pods of Baptisiu tinctoria at Washington, D. C.; Tachypterellus quadrigibbus Say in fruit of Cratægus mollis at Victoria, Tex.; Smicraulax tuberculatus Pierce in stems of mistletoe 
(Phoradendron faveseens); Tyethius sordidus Lechonte in Baptisia pods at Collecre Station, Tex.; Trichobaris compactn ('asey in pods of Datura stramonium at Paris, Tex.; and, furthermore, on Languria sp. in stems of Gaura sp. at Ballinger, Tex.

Cerambycobius cushmani Crawford. This parasite was reared in small numbers as early as 1906 in southern Texas from the boll weevil. It has been reared from Laria ochracea Schaeffer in pods of Tieire sp.: from L. sallaxi Sharp in pods of Tachellen furnesinne; from Arecerms fesciculatus DeGeer in fruit of chinaborries (Melin azderach) at Victoria, Tex.; and from Trichobaris texana in stems of Solanum rostratum.

('orambyeobrius sp). On February 2:3, 1909, a male of at sereen speeries of (crambyenthius was reared from the weevil in squares collected at Natchez, Miss., January 19.

PTEROMALIDA. PTEROMALINA.

Catolucens huneri ('rawford. This is the species which in all previons artiel (w on the boll weevil has been known as Catolacens incertus. Its hosts ats now known are Laria compressicornis Schacfier in pods of Acuan illinonsis: Tachypterellus quadrigibbus Say in fruit of Cratecyns spp.; Smicranlax tuberculatus Pieree in stems of Phoradendron favescens; Anthonomus æneolus Dietz in buds of Solanum splu.; Anthonemus albopitosus Dietz in seeds of Croton sp.; Anthonomus cugenii Cano in fruit of pepper (Capsicum spp.); Anthonomus heterothecip Pieres in heads of IIcterotheca subaxitlaris; Anthonomus nebulosus Le Conte in buds of Cratregus spp.; Anthonomus sigmatus Say in buels of dewherry (Rubus spp).); Anthonomus squamosus te ('onte in heads

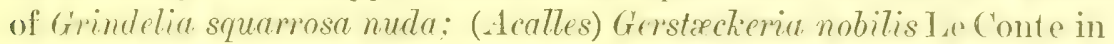
joints of Opuntia spp.; Zygoburis xanthoxyli Pierce in berries of . Ianthoxylum clava-hercutis.

Catolacens incertus Ashmead. This parasite is also very common and is known from a considerable number of hosts, among which are Laria cxifua Le Conte in pods of Amorple frutiensa: A piom decoloratum Smith in poods of Meibomia paniculata: Apron ariseum. Smith in pords of Phaseolus sple.; A pion niymum Smith in huds of Robinia psondacacia; Anthonomus albopitosus Dietz in seeds of ('roton spp.; Anthonomus aphenostephi Pieree in heads of Aphanostephus stirmobusis: Anthonomus fulves Le Conte in buds of Catlirrhoe involuerata: Anthonomus nigrinus Boheman in buds of solanum carolinense: Anthonomus signalus say in buds of strawberry (Fragaria virginiana); Autentes tcruipes Dist\% in buds of Galpinsia hurtwegi: ('cutorhynchus n. sp). in crown of Sclenin aurer: Baris cuncipennis ('asey in roots of Helenium tenuifolium and Calandra oryza Linnæus in corn 
Habrocytus piercei Crawford. 'This is a brilliant green parasite resembline fatolacems anthonomi Ashmearl. It is reared from the boll wereil mainly in the fall and from hibernat erl individuals in the spring. It has been reared from Laria compressicornis Schaeffer in pods of Acuan illinoensis. (See fig. 10.)

Lariophagus texanus Crawford. There is every evidence that this speries is a true parasite of the boll weevil, although it has not been positively reared by isolation from the boll weevil. On August 17 and 27, 1907, two specimens were reared from material collected at Hallettsville, Tex., August 13; on Aurust 19 and 23 three specimens were reared from cotton squares collected at Victoria, Tex. ; on August 29 three specimen; were reared from squares which were collected at Eagk Takke, Tex., on August 14. The Victoria lot was peculiar in that it fumished the first recorels of Cerambycolius cushmani Crawford, Spritochateis sp., and Eurytoma n. sp). This species is described as a

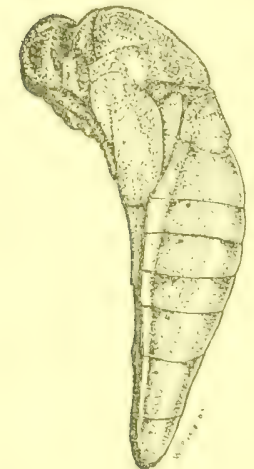

FIG. 10. - H abrocytus piercei: Pupa. Much enlarged. (Original.) parasite of (Bruchus) Laria prosopis Le Conte. It undoubtedly also attacks L. sallæi Sharp, which also breeds in the porls of huisache; furthermore, the species was reared from stem galls of Leucosyris spinosus containing Anthonomus ligatus Dietz.

Tetrastichus hunteri Crawford. This interesting new parasite of the boll weevil was first reared in the fall of 1908 from isolated parasitized individuals of the boll weevil collected at Natchez, Miss., by I. S. Smith. It is internal in weevil larvæ and pupæ and has even been reared from immature adults. A parasitized individual can easily be told by its brownish color and smoothening of the various segmental wrinkles. In more advanced stages of the parasite's development, the parasitized individual beremes a mere brown skin of parchment. This skin serves as puparium for the parasite. The developmental period is of considerable length in the fall. Specimens isolated in Norember do not mature until April or May. In 1908 it was found only at Natchez, Mis.s, and $\mathrm{X}$ [onroe, Lan., but in 1909 it was reared at a number of places in Louisiana and also at Arlington, Tex. This species gives an excellent example of the adjustment of native parasites to the boll weevil.

ICHNEUMONOIDEA. ICHNEUMONIDA. PIMLIN.E.

Pimpla sp. On January 27, 1909, a larva of this species was isolater from a weevil larra in squares collected at Nacogdoches, 'Tex. This became a mature female on February 23. 
BRACONID.E. SIG.ITIIIN.E.

Sirgalphus curculion is Fitch. Previous to the summer of $190 \mathrm{~s}$ the first record of rearing this species (see fig. 11) from the boll weevil was considered doubetul, but begimning in August it was reared repeatedly in material from Ruston and Nonoe, La., and Natrher, Miss. Its other host.s are Comotrachchus affinis Boheman in hirkory nuts; (onotruchelus teyans Bohemam in petioles of hickory at J allas, 'T'ex., and

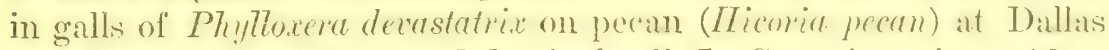
and Victoria, Tex.; Conotruchclus juglendis Le Conte in walnuts (.JugTans nigra); Conotrachelus nenuphar Irerbst in fruit of phum, peach, etc.; Tyloderma focedatum Say in stems of Onagme bremis at Washingtom, D. C. ; Trichobaris texana Le Conte in stems of Solanum rostratum; Trichobaris trinotata Say in stems of potato (Solanum tuberosum); and Zygobaris xanthoxyli Pierce in seed of Xanthoxylum clavaherculis.

Urosigalphus anthonomi Crawford has never been reared since the original records which were made at Brownsville, Tex.

Urosigalphus schwarzi

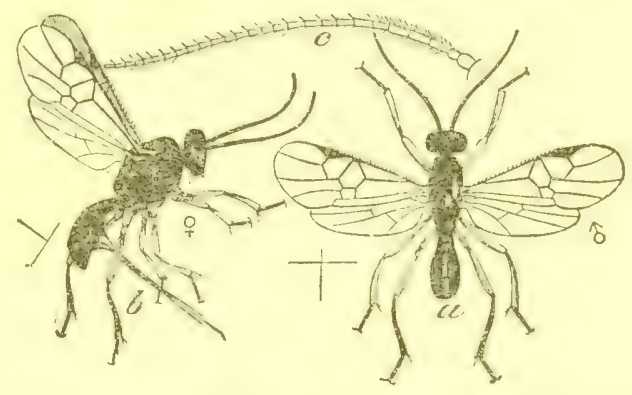

FIG. 11.-Sigalphus curculionis: $a$, Male; $b$, female; $c$, antenna. All enlarged. (After Riley.)

Crawford. This Guatemalan boll wereil parasite has never been reared in the United States.

Trosigalphus n. sp). At Arlington, 'Tex., in 1909, a single specimen was reared from an isolated cocoon.

BRACONINAS.

Micmbracon metlitor Say. ${ }^{1}$ This parasite (see fig. 12) still holds the lead as the most important boll-weevil parasite. Its other host relations are only partially discovered. The following hosts have been ascertained: Desmoris scapatis Le Conte in heads of Silcranthus mbiginosus; Smicrulax tubreulatus Pierce in stems of Phoralendron flaveseens; Anthonomus albopilosus Dietr in seed of C'roton spp.; Anthonomus euifenii Cano in fruit of pepper

1 Bracon mellitor Say is recorded by Girault (1907) as a parasite of the lesser peach borer (Synanthedon pictipes Grote and Robinson) and of the peach borer (Sanninoidea exitiosa Say). The gregarious habit of these parasites appears to prove that the determination was incorrect. Mr. F. E. Brooks, of West Virginia, has furnished the record of this species from Sanninoidea exitiosa and also from Craponius inzqualis Say at French Creek, W. Va. The determinations were made in the Bureau of Entomology. Dr. F. H. Chittenden states that he reared this species from the strawberry leaf-roller, A ncylis comptana Froelich (fragarixe Walsh and Riley), at Cabin John, MId., July 9, 1899. It is probable that all parasites of Lepidoptera determined as Bracon mellitor belong to some other species. The lepidopterous and coleopterous parasites are not distinguishable by structural characters, but are so different in habits that it is considered advisable to call the lepidopterous parasite Microbracon dorsator Say and the coleopterous parasite $\mathbf{M}$. mellitor Say. 
(C'rpsicum spp.); Anthonomus futvus Le Conte in buds of Callimhoe inmoncrain; Anthonomus squamosus tse Conte in heads of Grindelia stunarrose muda; Conotrachelus nenuphar Herbst in pearehes; Tyloderma forcolutum Say in stems of Onagra biennis; Craponius inxquatis Say in fruit of grape (Vitis spp.), and Baris sp. in roots of Ambrosia. sp.

\section{BIOLOGICAT, NOTES UPON THE PARASITES OF THE WEEVIL,}

A number of very interesting facts, which deserve mention in an economic hullet in, have been learned about the biology of the parasites.

\section{ABUNDANCE OF PARASITES.}

It is unusual for the parasites of the boll weevil to be found flying in numbers. Their work is in a general way quietly and unostentationsy done, but occasionally it is the privilege of the observers to see swarms of parasites hovering around the food plant of their

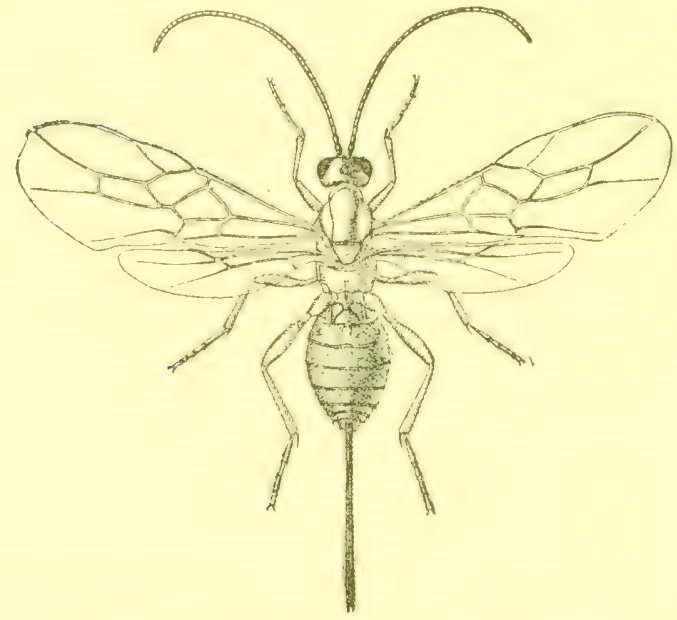

Fig. 12.-Microbracon mellitor: Adult. Much enlarged. (From Hunter and Hinds.) favorite host. At Clarendon, Tex., in August and September, 1905, $\mathrm{Mr}$. C. R. Jones and the senior author witnessed large numbers of Microbracon nuperus and Tetrastichus sp. hovering around the highly infested pods of Mentzelia nuda. The parasitism in pods gathered at this time was so high that much superparasitism by Tetrastichus upon theMicrobracon occurred. At Ruston, La., in October, 1907, the senior author saw Catolaceus

Tunteri flying in all directions and resting on the flowers and leaves of Ileterotheca subaxillaris and found a very high parasitism of Anthonomus heterothec by this species. In November, 1908, in this same field at Ruston, a very high pereentage of parasitiom of the boll weevil by ('atolaceus was found. In September, 1908, Mr. Hood saw many species of parasites around the flower heads of Vernonia at Camden, Ark. During the same month Mr. II. S. Smith found Catolaceus hunteri swarming on Croton capitatus containing inthonomus albopilosus. Such observations are very important because they suggest excellent sources for parasites to be used in introlution experiments or suggest foreing of the parasites to the boll weevil by the elimination of the host. 
FREQUENTATION OF NECTARIES.

'The feeding habits of adult hymenopterous parasites have long escaped ohservation, but within recent years the intensive study of parasites has proved that very little can be accomplished in the propagation of parasites unless they can be fed. In the case of the parasites of the boll weevil it is imposible for the adults to obtain 1uomishment from the host in which they are oripositing, as has been proven in the case of parasites of externally feeding insects. The host plant of the boll weevil, however, furnishes the desired fond. The nectaries of cotton are about as plentiful as those of any other plant. The majority of varieties of cotton have three large nectaries on the leaves and also have them on the outside and inside of the involucre, as well as on the inside of the flower. Frequent observations of cotton plants which were producing considerable nectar have enabled us to observe practically all of the parasites of the boll weevil, as well as all of the ant enemies and many other insects. Some of these insects which visit the nectaries are injurious to the cotton plant, but the majority seem to be beneficial.

The quantity of nectar secreted by various varieties of cotton is quite valiable. The variety which seems to secrete more than any other which has been observed is the Egyptian Mit Lffif. This variety is frequently surrounded by large numbers of beneficial hymenopterous insects, although at the same time it appears to be very susceptible to boll weevil attack.

HELIOTROPISM.

The majority of the hymenopterous insects which have been under observation in this investigation appear to be positively heliotropic. In general this tendency can be utilized in rearing-cage technique to induce the parasites to go into small tubes placed in the rearing loxes, from which they can be easily removed. It has been noticed in rearing cages in which there were growing plants with plenty of food, air, and heat, that the parasites sought the lightest portion of the cage rather than the plants which could give them some shade from the hot sun.

The activity of the parasites is greatest when the sunlight is most intense. Observations at the nectaries of the Exrptian cotton confirmed this. When the sun was shining the parasites were very active at the nectaries and flying around the plants, but when a choul passed orer they seemed to disappear entirely. On doudy days none of the Irmenoptera, except the most inclustrious bees and wasps, was to be found at the nectar. Trelease (1879) states that "the extrafloral nectar of the cotton plant is far more abundant during night and in the early morning than at any other time, and 
this is true whether we consider the involucral or foliar glands." The parasites probably frequent the nectaries during the morning sunlight hours and then are equipped to go about their other duties during the hottest part of the day.

In addition to these actual observations as to the preference of parasites there are other very strong proofs of heliotropism. It has been found that there is a decided increase in the parasitism of weevil stages in hanging forms exposed to the sum over those in fallen forms which are more or less shaded. It is also apparent that the fallen forms most exposed to the sun receive the greater amount of parasitism. Among the hymenopterous parasites there is only one at present which seems to prefer a moist shady place for its work. This is Telrestichus hunteri (rawford, which is an internal parasite.

SEXES.

A numerical study of the records of rearing of parasites from the boll weevil shows that in the majority of the species the males are relatively fewer than the females. The following table will show the pereentage of each sex and also the number of parasites upon which these percentages are based.

TABLE XIX.-Relative percentages of the sexes of boll-weevil parasites.

\begin{tabular}{|c|c|c|c|}
\hline \multirow{2}{*}{ Species. } & \multirow{2}{*}{$\begin{array}{l}\text { Total in- } \\
\text { dividuals. }\end{array}$} & \multicolumn{2}{|c|}{ Percentage of sexes. } \\
\hline & & Female. & Male. \\
\hline 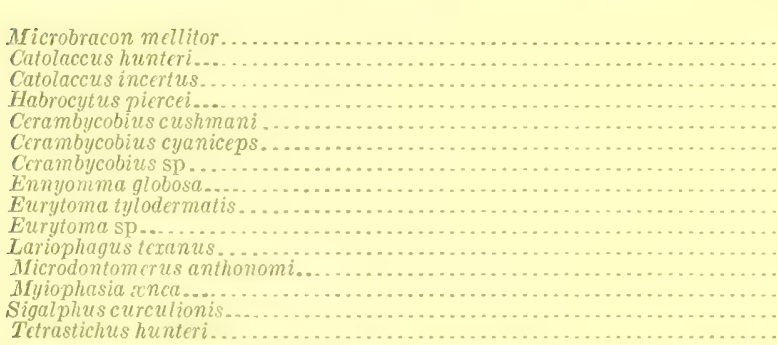 & $\begin{array}{r}980 \\
809 \\
429 \\
30 \\
64 \\
509 \\
1 \\
8 \\
433 \\
2 \\
2 \\
223 \\
2 \\
13 \\
41\end{array}$ & $\begin{array}{r}\text { Per cent. } \\
60.78 \\
78.37 \\
81.12 \\
100.00 \\
71.88 \\
70.34 \\
0 \\
37.50 \\
64.90 \\
50.00 \\
50.00 \\
81.76 \\
50.00 \\
61.54 \\
100.00\end{array}$ & $\begin{array}{r}\text { Per cent. } \\
39.22 \\
21.63 \\
18.88 \\
\ldots . . \\
28.12 \\
29.66 \\
100.00 \\
62.50 \\
35.10 \\
50.00 \\
50.00 \\
15.24 \\
50.00 \\
38.46 \\
\ldots . . .\end{array}$ \\
\hline
\end{tabular}

OVIPOSITION.

It has been found by numerical study of the large number of parasites collered during the last five years that whenerer the parasitism in a field reaches between 50 and 70 per cent there is a strong likelihood of reduplication, with resulting superparasitism. The exact records of superparasitism olutained in this investigation have been published in another article (Pierce, 1910). Parasites have no power of discerning the presence of another egg on the prospective hosts, and hence there occurs at times a tremendous duplication of energies. 

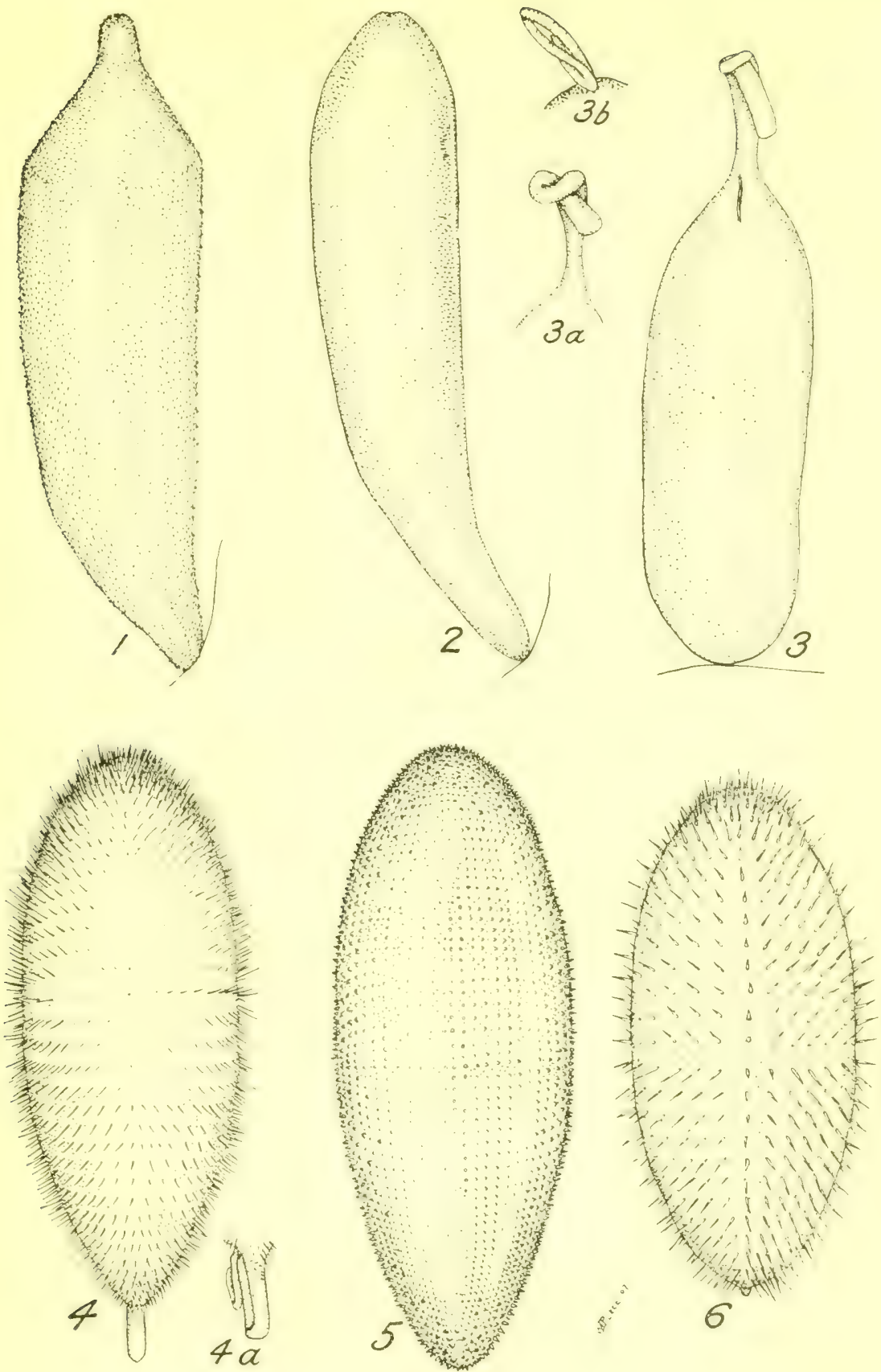

EgGS OF BOLL-WEEVIL PARASITES.

Fig. 1-Type II. Vicoduntomerus anthomomi: Calvert, Tex., August 23, 1907; color white; size 0.35 by $0.11 \mathrm{~mm}$. Fig. 2.- Type VI. Unidentified egg: Dallas, Tex., November 14, 1907. color white; size 0.45 by $0.19 \mathrm{~mm}$, Fig. 3,-Type 1. Cerctmbyerbius cyntecps: 3t, view from side; $3 b$, view from end; color white: size about $0.8 \mathrm{~mm}$. Fig, 4,-Type III. Eury-

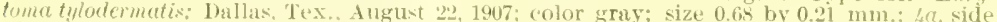
view of another egg. Fig. 5.-Trpe IV. Catolacens hinnteri: Dallas. Tex. August 22, 1907: color white: size 0.t2 by 0.22 mm. Fig. 6.-Type V. Unidentified egy; Glemmora, Lat. August 23,1907 ; color gray: size 0.44 by $0.11 \mathrm{~mm}$. (Original.) 


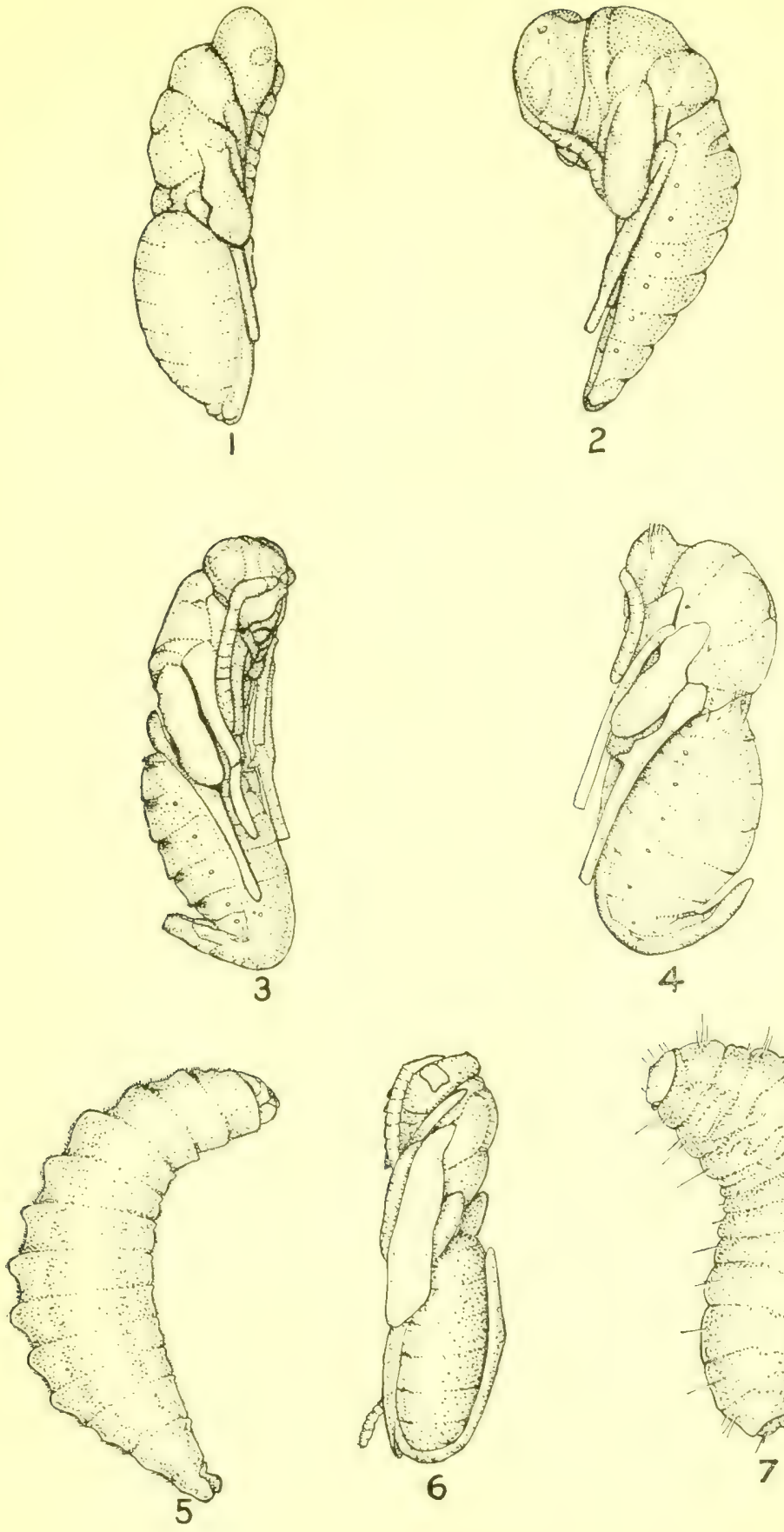

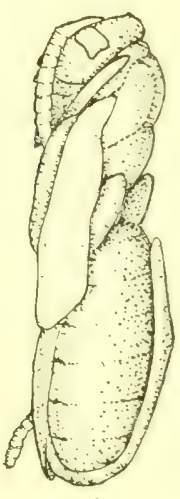

6

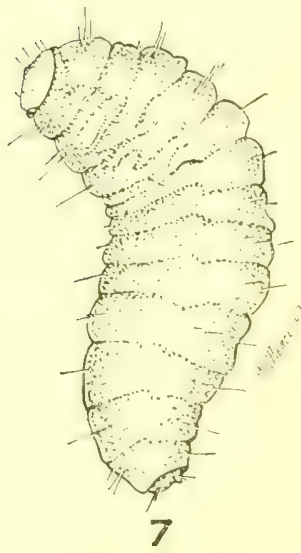

\section{PARASITES OF WEEVILS.}

Fig. 1.-Eumtoma tulotermatis, pupa. Fig. 2.-Catolaerus incertus, pupa. Fig. 3.-Crrambucobius cyonicess, pupa, Fig. 4.-Yicrofontomerus anthonomi, pupa. Fig. 5.-Larva of microbracon. Fig. 6.-Hicrubracon mellitor, pupa. Fig. 7.-Larva of chalcidoid. Much enlarged. (From Pierce.) 

It may therefore be possible that a parasite will visit the same square several times and oviposit. In general it maly be sald that as the primary parasitism of the bell weevil increases the superparasitism also increases, with the result that sometimes the parasitiom might, be enniderably increased if every egg reached a single host. The following instances will illustrate this. At Calvert, Thes. 41 stages were attacked by 44 parasites, although only 36.5 per went of the weerils were parasitized. If every parasite egg had reacherel a hest, there would have been 107.3 per cent parasitism. At Dallas, Tex, out of 309 weeril stages, 44.6 per cent were attacked by 216 palrasites. The possible parasitism was 699.9 per eent. Many other instanees of this kind eould be given, but these two cases illustrate the condition perfectly as it exists in many places during the fall of each year.

The time for oviposition apparenty differs for the various species. Microbracon mellitor, as a rule, oviposits before the boll-ivervil darva has constructed a cell, that is, several days before the flared square falls or dries. Eurytome tylodermatis appears to oviposit in squares on the plant after the normal time of falling and hence is more important in

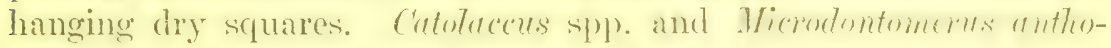
nomi favor fallen forms for oviposition. The chalcids generally oviposit after the weevil larva has formed its cell. Tetrastichus hunteri is most frequently found in fallen squares.

\section{THE DEVElophent OF THE PARASites.}

THE EGGS.

The eggs of the boll-weevil prarasites are all oblong-elliptis: and either smooth or sculptured. The eggs of several speries have at one or both ends a small tulie which is tied into a knot. Six types of egus of the boll-weeril parasites have been elosely observed and designated by number in the records of rearing. There are illustrated on Plate II. The eggs of all the boll-weevil parasites are placed in the weevil cell or on the larva or pupa and usualiy without injuring the latter.

Type I.-Type I is the egg of Cerambycobius cyaniceps. It was determined for the species by the use of a mica plant care in which the parasite was isolated with newly infested squares. This eyge is about $0.8 \mathrm{~mm}$. long, pure white, (roindrical, unseulptured, and with a narrow neck, which is twisted into a knot, probably by the ovipositor after the latter has released it. (Plate II, fig. 3.)

Type II.- This is the egog of Merodontomemes anthomomi, as shewn by the rearing of an isolated specimen. The color is white, the exger being distinguished by the shightly papillese seulpture and hy the nipple at one end. It measures $0.38 \mathrm{~mm}$. in length and $0.11 \mathrm{~mm}$, in breadth. (Plate II, fig. 1.) 
Type III.--This is the egg of Eurytoma tylodermatis, found by the isolation of a larva seen in the act of hatehing, collected at Dallas, Trex., Angust 2'?. The egre is dark-gray and thickly covered with spines. It measures $0.68 \mathrm{~mm}$. in length and $0.21 \mathrm{~mm}$. in breadth. The process at one end is frequently twisted. (Plate II, fig. 4.)

Type IV.-This egg was practically identified as that of a Catolaceus by isolation of specimens collected August 22 at Dallas, Tex. The color is white and the egg is covered with very small tubereles or papillar. It is $0.62 \mathrm{~mm}$. long and $0.22 \mathrm{~mm}$. broad. (Plate II, fig. 5.)

Type V.-This egg was taken only at Glenmora, La., August 23, on two weeril stages, and has not been identified. It is dark-gray and very spiny, but the spines are larger, longer, and sparser than in Type III. The length is $0.44 \mathrm{~mm}$, and the breadth $0.19 \mathrm{~mm}$. (Plate II, fig. 6.)

Type VI.--This new type was discovered November 14 at Dallas, Tex, and has not yet been identified. There is no seulpturing whatever. It is pure white. The length is $0.85 \mathrm{~mm}$. and the breadth $0.19 \mathrm{~mm}$. (Plate II, fig. 2.)

THE LARY F。

The larva of the boll-weevil parasites live as readily on dead food as on fresh food. The hosts generally die within a rery short time after the larvæ begin attack. The larvæ have been found pretty well grown with dry weevil larvæ as food. They have been found on weevil larvæ and pupæ indiseriminately and several times under the elytra of teneral or unemerged alults. Just before transforming from the larva to pupa there is considerable meconial discharge. The majority of the boll-weevil parasites are external feeders, but the larre of Myiophasia atne, Ennyomma globose, and Tetrastichus hunteri are internal feeders. These larvæ kill the host in a short time, its skin becoming shriveled and forming a perfect puparium for the parasite. Pupation takes place within this skin. (Pl. III, figs. 5,7 .)

Pupation.-All the chalcidoid parasites have naked pupæ. The braconids usually form silken cocoons of characteristic size, shape, mesh, or color. The cocoons of Microbracon mellitor are very variable in size, color, and consistency, so that they appear almost to belong to diflerent insects. The cocoons of Sigalphus curculionis are generally of a rather bright yellow and with very fine silk. The pupal exuvium of the various species of chalcids and braconids is sufficiently characteristic to enable a skilled observer to determine the species after the parasite has left. (Pl. III, figs. 1-4, 6.)

Rativity of development.-It is rather difficult to make an accurate study of the developmental period of parasites, especially when every adult parasite that matures under observation must be saved, if 
possible, for further experimentation or for determination. It is inadvisathe to isolate many of the parasites until the lawra is partially developed, as the isolation seems to dry out both food and larva. In the study of the parasites all those in the same stage were plabed on the same tray. When they passed to the next stage in development they were transferred to another tray. In this mammer an alectlrate record was kept of the development. In order to determine the total length of the breeding period it seems best to take the total period from the collection of the material to the maturity of the late specimen and add a plus mark $(+)$ to this figure. 'The total period can hardly be more than 2 or :3 days longer than the longest period thus obtained, as the egg period is very seldom more than 3 days. To oltain the exact length of the pupal period, the maximum period is taken to be the longest time from the observation of a fresh or newly-formed pupa to maturity, and the minimum time is talsen to be the shortest period from the observation of the grown latrat to matturity. IIaving thus aceurately defined the pupal stane, the relative limits of the egg and larval stages are obtained by subtracting the pupal stage from the total derelomental period. Tiable XX, which follows, presents all of the available data as they have been reduced in this manner to show the length of devclopment of the various stages. It will be seen that most of the species pass their entire developmental period in from 20 to 30 dabys between . Tune and Oetober 15, but that after the middle of October the developing stages are caught by the cold weather and the development is suspended until spring. Thus, it is noticeable that parasites becoming larva in early October and November have a short lanval periox of probahly less than 20 days, beeming pupa before the cold wave and passing a pupal period of about 150 days. Parasite larvæ which hatch a little later are caught in the larval stage and hibernate thus for from 120 to 150 days, then becoming pupæ and maturing in from 15 to 40 days. It will be noticed that Microbracon mellitor, Euryfomatylodermatis, and the two species sif Catolacens have short developmental periods during the summer, while the species of Cerambycobius have a little longer period. It will be noticed that Hethroeytus piencei has only appeared in the fall of the year. This species has been recorded four years in suression and never before Octobes. On the other hand, Hifrodontomerns anthoment secms to be almost exclusively a summer parasite, having never been recorded after September. Of course the species of which we have records throughout the breeding season are the ones most important. This statement is borne ont by the figures on the relative numbers and importance of the different species. 
TABLE XX.-Lengths of developmental periods of the boll-weevil parasites.

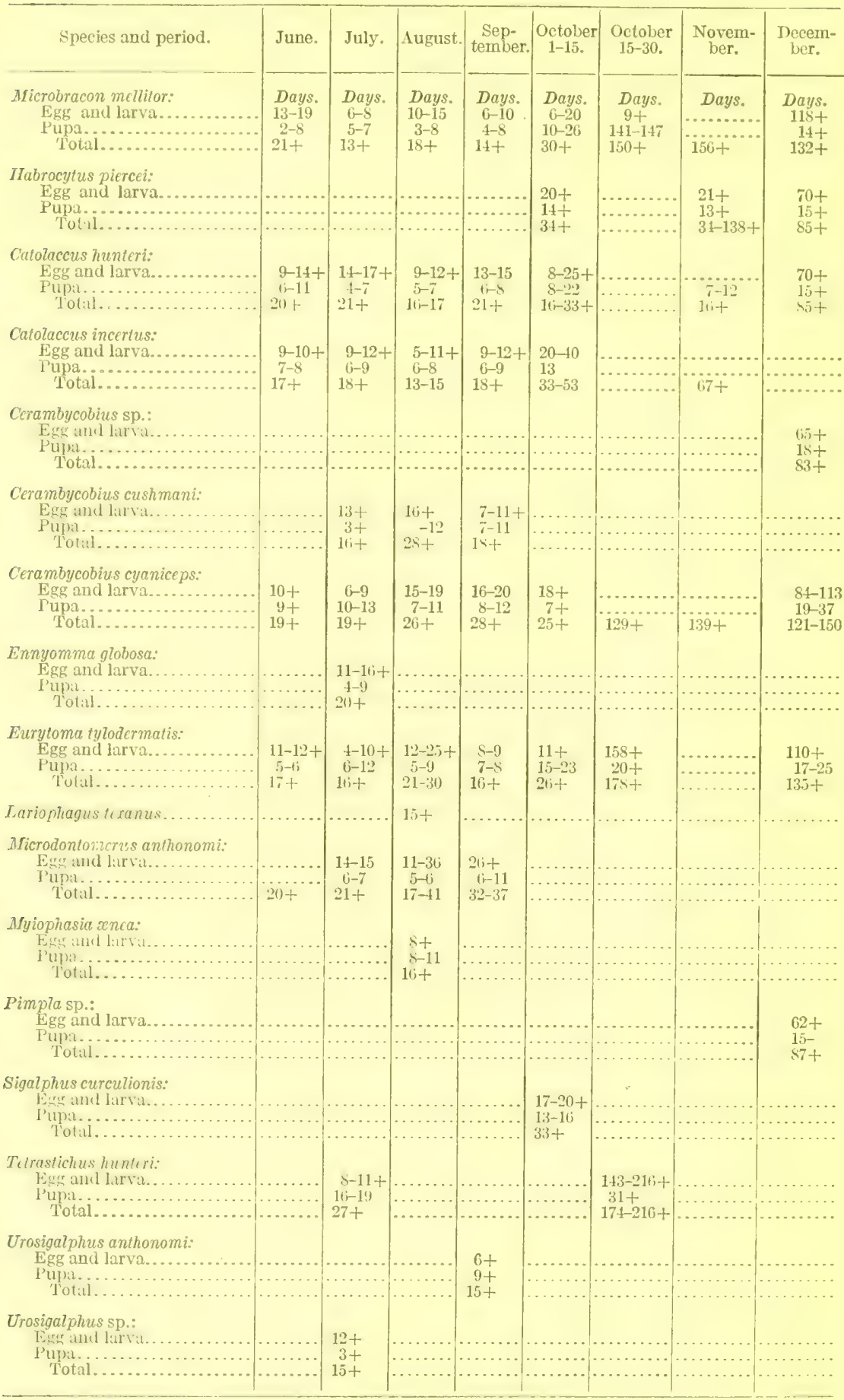




\section{THE DISTRIBUTION OF THE PARASITES.}

Parasites of the boll weevil have been recorded from every part of the territory so far invarled. The records are so mumerous that we are able to show statistically which are the most important parasites of the weevil. The following list gives the specios in their numerical rank for the entire period from January 1, 1906), fo January 1, 1910, giving only the number which were accurately dotermined foreach species. The first seren species are the mostimport ant, as has been shown in almost erery section of this report. 'The last nine species may be considered as more or less adventitious ur alcidental. These species may possibly never be recorded again, or, on the other hand, they may become in the near future among the nore important parasites. 'This very event has happened in the casce of three or four of the other more important species. L'p 101906 only four of the first fire in this list had been recorded from the boll weeril. The other species have been added since and some of them will become very important as the weevils enter the moister wooded regions of the East.

'TAвте XXI.-Numerical rank of the parasites for the entire period, 1906 to 1910.

\begin{tabular}{|c|c|c|c|}
\hline Species. & $\begin{array}{l}\text { Number } \\
\text { of } \\
\text { records. }\end{array}$ & Species. & $\begin{array}{l}\text { Number } \\
\text { of } \\
\text { records. }\end{array}$ \\
\hline 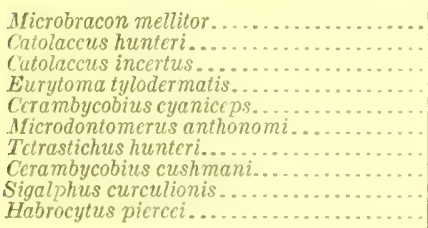 & $\begin{array}{r}2,147 \\
1,094 \\
578 \\
575 \\
574 \\
302 \\
168 \\
76 \\
37 \\
36\end{array}$ & 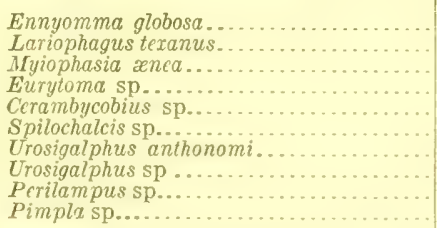 & $\begin{array}{r}35 \\
8 \\
4 \\
1 \\
1 \\
1 \\
1 \\
1 \\
1 \\
1\end{array}$ \\
\hline
\end{tabular}

A study of the value of these parasites by years has shown that the majolity of the species had not occupied the same rank in two successive years. The aceompanying diagram (fig. 13), wiving the rearly rank of the boll-weevil parasites from 1906 through 1909, shows that in each year new parasites were recorded and that in some (aises these parasites continued to attack the weevil. Microbracon mellitor appears to vary but little in importance in different scasons, while Catoleceus liunteri shows inereasing importance year hy year. Some of the ot her parasites of considerable importance appear ext remely variable in their relative rank. It will be noticerl that Habrocytus piercei has occupied the ninth place three years in succession and is now in eighth place. This parasite occurs in small numbers, but may at any time become a leading parasite in Louisiana and Mississippi. In addition to giving the yearly rank of the species this diagram also shows the proportion of the sexes observed each year. 
In order to show the regions in which the various species are of createst importance, the accompanying map) (fig. 14) is presonted. This slows that while Microbracon metlitor has yielded more individuals than the other species, it is the predominating parasite in by far the Jarere proportion of the infested territory. It can also be seen that mich more can be expected from the other parasites as the weevil moves castwar! into their territory. Nicrodontomerus anthonomi is quit important throughout the central black-prairie region of 'Texas. Euryfomatyleltmatis is more important in north-central Texas and also in the coast region of Texas. Combucobius cushmani is charac-

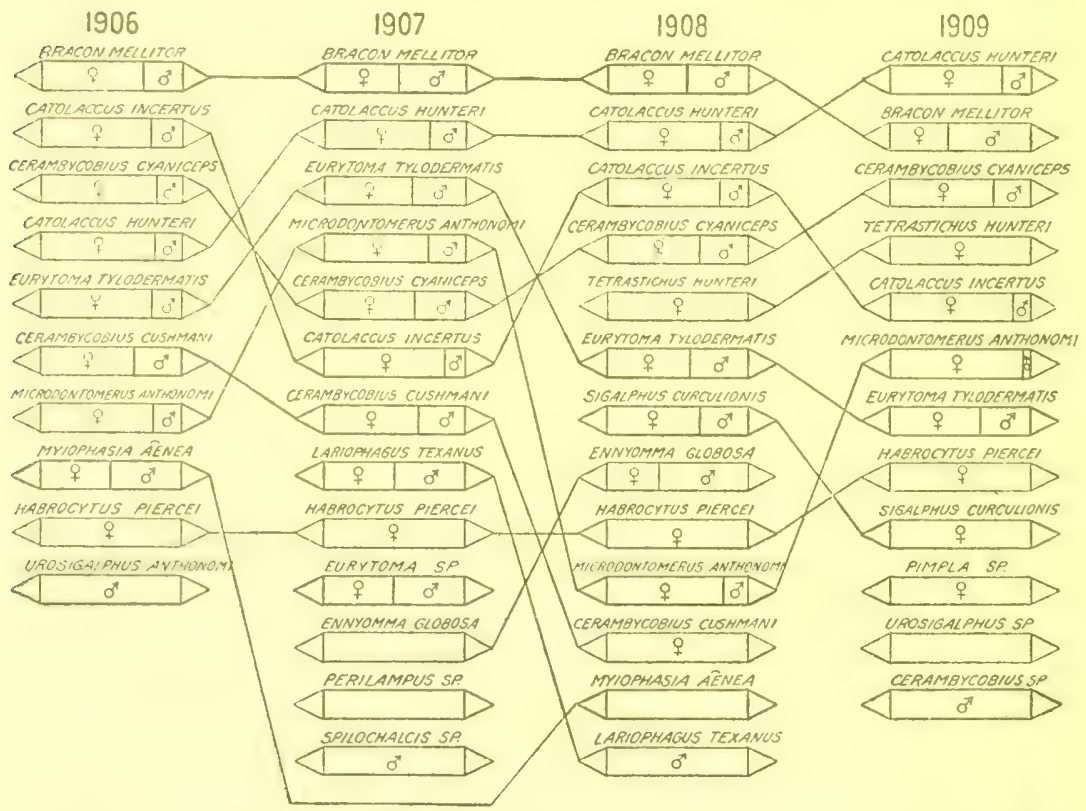

FIG. 13.-Dlagram 1llustrating yearly rank of the boll weevll parasites, 1906, 1907, 1908, and 1909. (Original.)

teristic of the counties grouped around Victoria County, Tex., but a few specinchis have been reared from the boll weevil at Alexandria, La., by Messrs. Cushman and Jones.

\section{THE PARASITE SEASONS.}

For the convenience of this work on parasites of the boll weevil, the year has been divided into definite parasite seasons corresponding with certain groups of conditions. The year opens with the hibernation period well underway. In so far as the parasites are concerned those which hibernate as immature insects mature renerally about the middle of March. This marks the end of the hiber- 
nation period or winter season and the opening of the spring season. From March until the middle of June or sometimes July there are no cotton squares for the weevils to breed in. Consequently the parasites are olliged to seek other hosts. The summer season is defined as beginning with the production of squares in which the weerils and their parasites may breed. Thus this season continues until squaring ceases - that is, until late in the fall when cotton is killed by frost and is succeeded by the winter season. However, we frequently distinguished a fall or postmigration season, which begins with the first

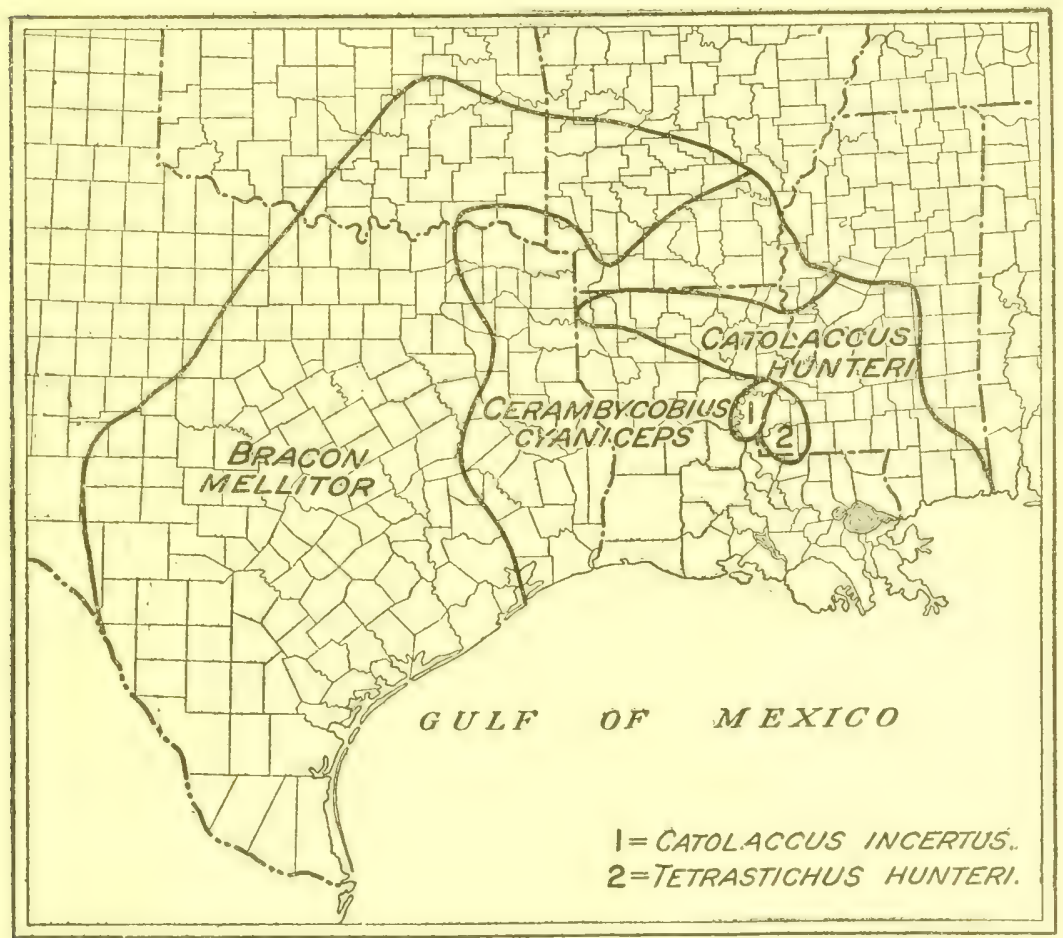

FIG. 14.-Map showing the distribution of the more important parasites of the boll weevil. (Original.)

attack of weevils upon the bolls in August and ends with the heavy frosts in October or November. The fall season is also characterized by a renewed growth of squares.

I. THE HIBERNATION OR WINTER SEASON.

The most important parasites which winter as immature stages upon the boll weevil are Microbracon mellitor, Catolaccus hunteri, C'crambifcobius cyaniceps, Eurytoma tylodermatis, Tetrastichus Tunteri, and IIabrocytus piercei. The last two species are characteristic of winter examinations in Louisiana and Mississippi. The predatory 
colcopterous larve IIydnocera pubescens LeConte and II. pallipennis Say are very frequently found hibernating as larva in the bollweevil cells of in the cocouns of Micobracon mellitor. The stage in which these rarious parasites patss the winter is given very concisely in the bable of the developmental periods (Table XX) in section 7. During fanuary, 1910, Mr. Irood repeatedly found Eurytoma tylodermatis and Catolaccus hunteri hibernating in dry cotton squares and bolls and especially in hanging moss at Mansura, La.

\section{THE SPRING SEASON.}

It has been demonstrated that there is a definite period between the hibemation season and the first infestation of squares, catending fiom the middle of Warch to the middle of June. What hatppens to the parasites duning this perion is of considerable inportance and a great anount of work has been done in the seareh for intermediate hosts.

In the case of Catolaccus hunteri the question was very satisfactorily answered. At Richmond, Tex., a large number of dewberry

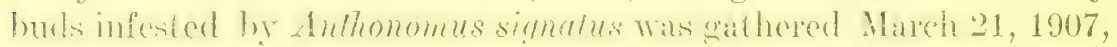

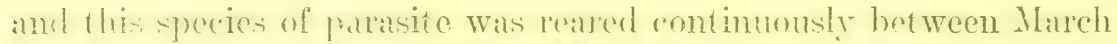

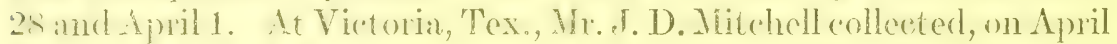

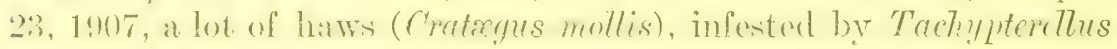
quadrigibbus, and on May 7 he reared this species of parasite. Investigations as to the distribution of these weevils added to the formerly known records of Anthonomus signatus in dewberry buds: Natehitehes and fhrreport, I Lil.; Texarkana, Ark.; Nuskogee and Ardmore, Okla.; anel Thinity, Richmond, Taro, Dallas, and Marshall, Tex. Trechyptrollus quatrigibluss was found breeding at Shreveport and Natchitoches, La., and Victoria, Tex.

At Dallas, 'Tex., the buds of Galpinsia hartwegi were found to be infested by Auleutes tenuipes as early as April 24. This species is a host of several species of Catolaccus. 'The buds of Caltirrhoe involucrata were found at Dallas to be infested by Anthonomus futvus as carly as April 1, and on the same date inthonome acneolus was first observed to be breeding in the buds of Solanum torreyi. Solanum ceragmifolinu, with thlhomomus ancolus both in its buds and in the fungus leaf-enll , and solimum rostratum with this weovil in the buds, appeared cal! in April. Nll of these plants continued susceptible to weevil work up to the cud of the ypring perion, or unt il cotton began to square. Numerous specimens of Catolaceus were reared from the Solanum-infesting species of Anthonomus.

Myiophasia ænea was reared April 11, 1907, from Conotrachetus cleygns in sills of Phylloxera decastatrix on the petioles of IIicoria 
pecan, collected April 2, 1907 , at Victoria, Tex., and was reared Jume 5, 1907, from material collected May 4 at Dallas.

Sigalphus curculionis was reared in consilerable numbers between April 28 and May 7, 1907, from Comotrachelus nemuphar in plums gathered at Texarkana, Ark., Narch 26; and betwoen 1pril 29 and May 17, 1907, from Conotrachehes cleyans in galls of Phylloere devastatrix on pecan, collected at Victoria, Tex., April 2); also between June 5 and 14,1907 , from the same species in material collected at Dallas, Tex., May 4.

Cerambycobius cyaniceps was studied very carefully at Victoria, Tex., by Mr. J. D. Mitchell during the winter of 1909-10 as an enemy of Trichobaris texana in stems of Solanum rostratum, and of Lixus scrobicollis in stems of Ambrosia trifida. Mr. T. T. Iolloway conducted experiments in longevity by fecting sugared water to the parasites. Emergence began, in the lots of Trichobaris, on February 1 and continued until April S. The last parasite lived until May :31. The total period of activity was 119 days and the average period lasted from March 11 to April 1. The longest record of longevity was 71 days and the average 21 days. Emergence began from the lots of Lixus on March 2 and continued until March 24. The last parasite lived until May 11. The total period of activity was 70 days and the average period was between March 13 and April 4. The longest record of longevity was 67 days and the average 22.

Eurytoma tylodematis was reared from the same lots and treated in the same mamner. Emerenee began from the lots of Trichobaris on February ;3 and continued until March 21. "The last parasite lived until April 30. The total period of activity Was s6 days and the average period was between March 10 and March :30. The longest record of longevity was 42 days, and the average 20 days. Emergence began from the lots of Lixus on February 22 and lasted until $\Lambda$ pril 17. The last parasite lived until June 1. The total period of activity was 99 days and the average period lasted from Marele 16 to April 11. The longest record of longerity was 79 days and the averace 26 days.

III. THE SUMMER SEASON.

The first boll-weevil parasites of the year are reared late in Maly or early in June in southern Texas, but in a very short time squares are forming all over the entire cotton belt and parasites may be found everywhere in small numbers as the summer progresses. The percentage of parasitism increases rapidly and erenerally becomes rery ligh after August 1. Most of the important parasites may also be found on their normal summer hosts.

$$
\text { 16844은 Bull. } 100-12-5
$$


About the middle of August squares commence to fail, and few squares are to be found by September 1 . This condition may be said to begin the fall season, when the parasites are largely obliged to seek other hosts or to attack the boll weevil in bolls.

IV. THE FALL OR DISPERSION SEASON.

('oincident with the decline in square production is the beginning of the boll-weevil dispersion which extends into new territory around the entire periphery of the infested region. In the fall there is a new growth of squares which furnishes food for the weevils before entering libernation and also furnishes an opportunity for very high parasitism just preceding hibernation. It is during this season that parasite swarms are recorded and hence this is a very critical tine for obtaining and transferring desirable parasites to new regions. During this early fall scason there are several very important ways of propagating the parasites alrealy present in the vicinity, as will be shown later. The fill seasom of the year closes abruptly with the first killing frost, for this erisis precipitates the hibernation period.

\section{AD. USTMENT TO NEW HOSTS.}

It is a very striking fact that the continuously breeding boll weevil is attacked by parasites which in many instances attack normally weevils having but a single generation annually. Some of these parasites attuck one host after another throughout the entire breeding scason and may be found in activity at all periods except during hibernation. This condition is well illustrated by the accompanying diagram (fig. 15) giving the seasonal rotation of Catolaccus hunteri and Cerambycobius cyaniceps. Whether these parasites were originally single-generation species like their hosts is a question we can not now decide, but we now know that they lawve become adapted to many species. This fact can be most easily proven by reference to the list of hosts of the boll-weevil parasites given in the second section of this part (p. 42). It appears possible that the constantly changing factors of nature cause the various species to be continually adjusting their habits to new environments and new hosts. In other words, the groups of parasites from which the most available enemies of a new or introduced species may be obtained are those groups in which the parasitic habits are the most variable. A parasitic species that is as readily at home on a stem weevil as on a bud or seed weevil is probably able to attack many different species.

The most striking example of the adjustment of new parasites was furnisher in 1907. A lot of hangine squares collected by Mr. J. D. Nitehell on August 5, 1907, at Victoria, Tex., on a field known as the Haskell field gave a pereentage of 61.5. There was something so 
striking about the nature of the record that Mr. ('ushman was sent immediately to Victoria to study the surroundings of this field and report upon the possible reasons for the high pereentage of parasit ism. Mr. Cushman reported after considerable study that there were only two factors which, it seemed to him, might have an influence upon the parasites of the boll weevil. The first factor was the eomplete lack of fruit upon the huisache trees (Tuchelline furnesiana) which is the normal food of Larin sallxi. The seeond factor noticed was the absence of flowers on the Catlimbe immotucrutu, the lenst of Anthonomus futvus. Mr. Cushman reasoned that the point would be proven if we should rear from the boll weevil some of the charracteristic parasites of either this or the other species. As a result of rearings from the material collected in this fich, the principal parasite was Microbraenn mellitor, the typieal bell weevil parasite, but a

SEASONAL ROTATION OF hOSTS BY CATOLACCUS HUNTEAI CRAINFOFO.

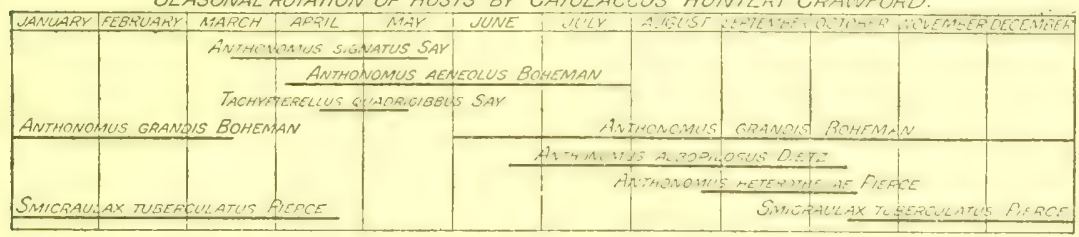

SEASONAL ROTATION OF HOSTS BY CERAMEVCOBIUS CYANICEFS ASHNEAD?

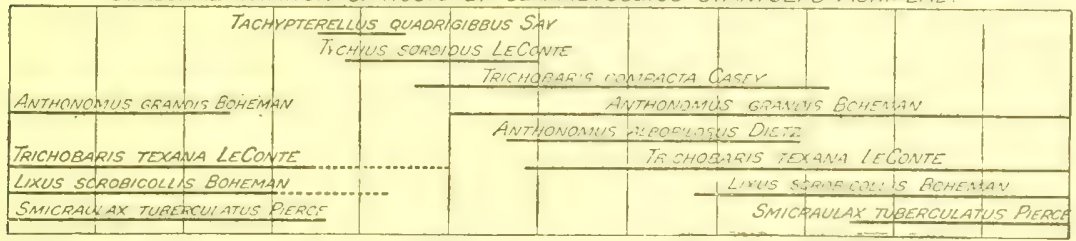

Fig. 15.-Diagram fllustrating the seasonal rotation of hosts of Catolaccus hunteri and Cerambycobius cyaniceps. (Original.)

species which is also a typical parasite of Anthonomus futerus. It is probable that the latter species furnished some of the Mierobracons for this infestation. The next most important speries was C'erambycobius cushmani, a typical parasite of Larict saltai and of Aræcerus fasciculatus which breeds in the fruit of the chinaherry tree (Melia azederach). In addition to this species, this sume ficld vichled 3 other new parasites of the boll weovil, 2 of which are known to be parasites of the Laria. These were Eurytoma sp., S pitoctateis sp., and Lariophagus texanus.

To illustrate the divergence of habits among parasites the host relations of catolaceus incertus may be cited. This parasite attackis several species of Larie. (Bmechess) which are internal seed eaters and pupate in their feeding eells: such weevils as Zugoburis artuthoxyli and Autcutes temeipes, which are seed or bud feeders and pupate in the 
ground; and Anthonomines, which dwell in buds (Anthonomus grandis), in flowers ( 1 . aphanostephi), and in hard seed ( 4 . albopitosus). But it draws the line apparently at stem dwellers and is replaced by Veocatolaceus tylodemixe on Tixus, Tyloderma, and Ampeloglypter. Cerambycobius cyaniceps is as much at home in a stem as in a bud, and so also are Eurytoma tylodermatis and Merodontomerus anthonomi. The Braconicla appear to be more particular as to food but the most noted of all, Microbracon mellitor, has no preferences between stem dwellers and bud dwellers.

Thirteen miles southeast of Yazoo ('ity, Miss., on November 1, 1909, the senior anthor found an isolated artifcial foeus of infestation by the boll weevil over 30 miles from any infestation of the same age and 20 miles beyond the regularly infested region. Out of 8 squares picked, containing 5 stages, 1 parastized stage was found.

\section{BEETLES WHICII PREY UPON TIIE BOLL WEEVIL.}

The attack of the insects predatory on the adult boll weevil is purely accidental. They may be very numerous, but the only ones recorded and verified are Evarthrus sodalis Le Conte and another species of the same genus. There are, however, several insects which have an actual value through their established habit of either breeding in the square upon the boll-weevil stages or of entering the square and consuming the weevil. We shall refer to four of them.

Hydnocera pallipennis Say. A single beetle of this species was reared April 6, 1907, after 183 days in its cocoon, and over 214 days isolation in the rearing tube. It was collected in a boll-weevil cell at Waco, Tex., August 28, 1906. The cocoon is very finely threaded, loosely woven, and only single layered. The stage of the beetle can easily be observed at any time.

Hydnocera pubescens Le Conte. This clerid is a very common breeder in the weevil cells. Its larva have been found not only feeding upon the various wrevil stages hut have been taken frequently from Microbracon cocoons which they have entered at a much younger stage.

C'athartus gemellatus Duval. 'This cucujiul bectle is both a predator' and a scavenger, its larve being frequently found, however, feeding upon boll-weevil stages which they must have killed.

Chautiognathus spp. The larvæ of these lampyrid beetles are very common in the squares and bolls of cotton in Lonisiana and Mississippi. In one instance undoubted proof of the attack of such a larva upon one boll-weovil larva was recorded. Many other very suspicious observations were made but no definite proofs found. 


\section{LEPIDOPTEROUS LARVE WHICH ARE INCIDENTALLY PREDATORY} UPON THE BOLL WEEVIL.

Alabama argitlacea IIübner. The cotton leaf catcrpillar is dist inctly ail enemy of the boll weeril and of considerable importance. When it defoliates a cotton field a month or more before the frosts it often destroys immature weevils in the cotton squares and cuts on' the entire food supply of the adult weevils remaining. These weerils maty be alble to suspend their activities and begin hibernation but it is woll known that weevils entering hibernation early in the fall can soldom survive a long hard winter, or live until cotton is up in the spring. Those that can not hibernate cither die of starvation or rise in flight to seek cotton elsewhere and may perish in the eflort. It is presumed that a very high pereentage of flying weevils fails to find cotton.

The leaf worm is attacked by 18 predatory bugs, 16 predatory beetles, 6 predatory wasps, and the following ants: Dorymyrmex pyramiens fueves Mccook, Forctius maccooli Emery, Solenopsis geminate Fabricius (these three ants are enemies of the boll weevil) and Ifonomorium curbonarium Smith. Ten hymenopterous parasites and one hyperparasite are known, and in addition the leaf worm is attacked by a predatory fly and by two parasitic flies.

\section{ANTS WHICII PREY UPON THE BOLL WEEVIL.}

\section{HYMENOPTERA. DORYLIDE.}

Eciton (Acamatus) commutatum Emerv. This ant was taken by Mr. ('. R. Jones at Beeville, 'Tex., attacking the boll-weevil latra' in squares. Dr. W. M. Wheclor states that it is commonly parasitized by a round worm of the genus Mermis.

PONERID正.

Ectatomma tuberculatum Olivier. The "kelep," or so-called Guatemalan ant, is a native of Mexico and ('entral America. Like all ot ther' ponerids it is slow in action. The winters have proven too severe for any of the imported colonies. The rate of development is so slow and the movements of the adults are so sluggish that little could be hoped for from this species exen if it could become accelimated in this country.

MYRMCIDÆ.

Cremastogaster lincolata (Say) var. clara Mayr. This ant is also an enemy of the boll weevil, having been recorded attacking immature stages at Dallas, Tex., by Dr. IY. E. Minds. It has frequently been seen in the rearing cage carrying ofl' insect prey. 'The species lives 
in hollow stems, sticks, and galls and is commonly seen at the nectaries of cotton or attending aphides, membracids, etc. Prof. F. E. Brookis has recorded this ant as an enemy of Iletiothis obsoleta, the cotton bollworm.

Solenopsis geminata Fabricius. The "fire ant" (fig. 16) is very common in T'exas cotton fields, where it is always an enemy of the boll weevil, as well as of the cotton bollworm (Hetiothis obsoleta) and the cotton leaf worm (Alabama argillacea). In Louisiana, Arkansas, and Mississippi it is very seldom seen in cotton fields, except in southern Louisiana, where unfortunately it is in danger of extermination by the Argentine ant, Iridomyrmex humitis Mayr. This species divides credit for the greater part of the ant control of the boll weevil with the other species of Solenopsis, two species of

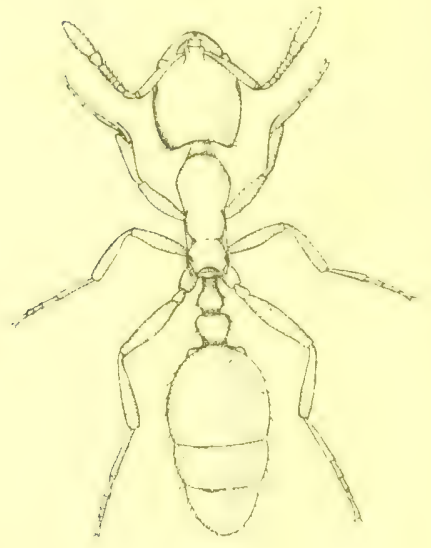

FIG. 16.-The "fire ant" (Solenopsis geminata), an enemy of the boll weevil: Worker. Enlarged. (From Hunter and Hinds.)

Monomorium, and with the various species of Pheidole. Its nests are placed in the cotton fields, generally near the base of the plants, and from these the foragers go out in all directions in search of food. The workers have learned to detect the presence of the boll weevil in the squares and in a short time can effect an entrance into the weevil cell from which they either draw the weevil bodily or convey it in parts to their nests. This ant is sometimes found on the plant, but most commonly it does its work on the ground. The species is parasitized by (Pseudacteon) Plastophora crawfordi Coquillett at Dallas, 'Tex.

Solenopsis molesta Say (debilis Mayr). This minute ant was taken in the act of attacking a boll-weevil larva by Mr. Cushman at McAlester, Okla. This species and the next are so similar in appearance that they may be easily confused. Prof. F. E. Brooks has recorded it as an enemy of Craponius inxqualis.

Solenopsis texana Emery. This minute ant is a common enemy of the bull weeril in 'Texis, Inuisiana, and Mississippi. "The entrance holes are very minute, but sometimes the ants enter the squares in great numbers. On October 31, 1907, at Thornton, Tex., Mr. ('ushman found s.) indiviluals attacking a weevil larva in a single square. It is mentioned in the investigation records as attacking the weevil at Mlexandria and Monroe, Lab., and Cuero, Lampasas, and Llano, Tex. It is also recorded as an enemy of Hetiothis obsoleta.

Monomorium minimum Buckley. This common house ant (fig. 17) is a very valuable enemy of the boll weevil and is common in cotton 
fields. It is recorded in the Dallas collection as attacking the boll weevil at Llano, Lampasas, Albany, IIenrictta, Arlington, and I)allas, Tex., Ruston, La., and Roxie and Port Gibson, Miss. The species has been taken attacking the immature stages of Trichobaris compacta, Anthionomus albopitosus, and Anthonomus futcus. It generally at tacks these weevils as well as the boll weevil on the plant, entering the infested bud or square in search of its food.

Monomorium pharaonis L. This cosmopolitan house ant (fig. 18) is another of the most important boll-weeril enemies, being rery

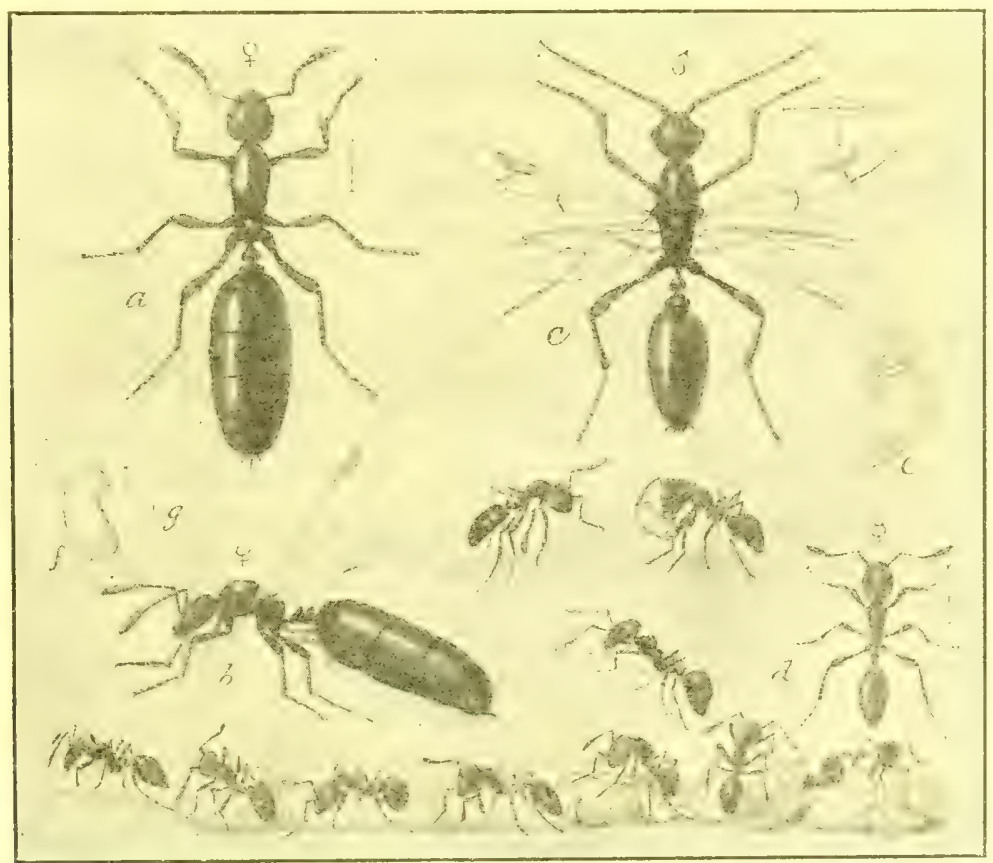

FIG. 17.-The little black ant (Monomorium minimum), an enemy of the boll weevil: $u$, Female; $b$, same with wings; $c$, male; $d$, workers; $e$, pupa; $f$, larva; $g$, egg of worker. Enlarged. (From Marlatt.)

abundant in the cotton fields of certain sections. It is represented in the Dallas collection as attacking the boll weevil at Victoria, Tex.; Fosters, Ruston, and Monroe, La., and Camden, Ark. It also attacks the weevil on the plant. In southern Louisiana it is being exterminated by the Argentine ant (Iridomyrmex humilis).

Pheidole sp., near flavens. At Arlington, 'Tex., August 31, 1908, Mr. Cushman found abunitint evidence of the control of the boll weevil by this species. It attacks the weevil larvæ both on the plant and on the ground. 
Phridote crassicomis Emery. At Lampasas, Tex., September 23, $190 \mathrm{~S}, \mathrm{Mr}$. ('ushman found this ant a very abundant enemy of the boll weevil.

DOLICHODERIDAE.

Forelius maccooki Forel. At Beeville, Tex., August 13, 1906, Mr. C. R. Jones found a high mortality of the boll weevil due to this species. Dr. Wheeler has recorded the fact that this ant prefers bare, dry gromed for its nests. The species also attacks Alabama argillacea and IIctiothis obsolele. On Scptember 7, 1908, at Dallas, Tex., Mr. F. C. Bishopp took specimens in the act of attack, and September 21, 1908, Mr. ('ushman took other's at Llano, 'Tex., attacking the weevil.

Dorymyrmex p!ramicus Roger, the "lion ant," protects solitary tree cotton from the boll weevil in Cuba (Schwarz, 1905).
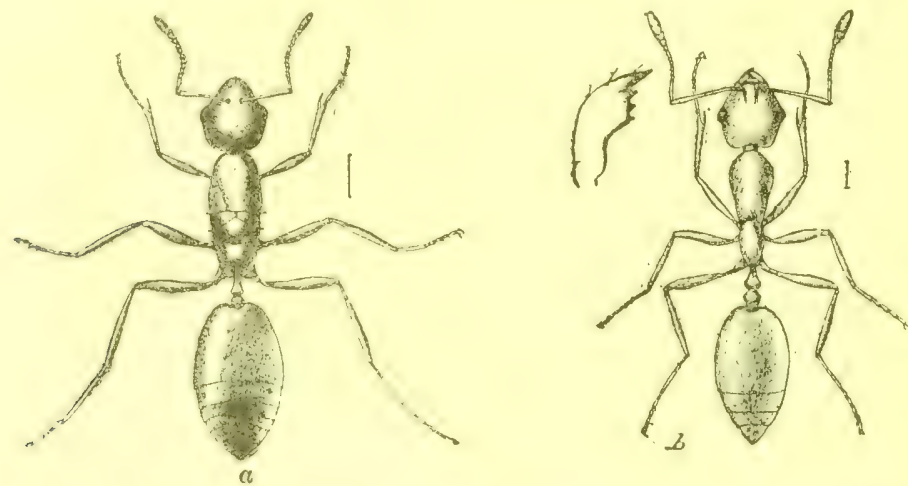

FIG. 18.-The little red ant (Monomorium pharaonis), an enemy of the boll weevil: $a$, Female; $b$, worker. Enlarged. (From Riley.)

Dorymyrmex pyramicus (Roger) var. flavus McCook. 'This common ant of the cotton fields has only once been taken as an enemy of the boll weevil, namely at Texarkana, Tex., by Mr. R. C. Howell, but its abundance would make it a very important species if it should develop a fondness for weevil larvæ. It is an enemy of Alabama argillacea and Heliothis obsoleta.

Iridomyrmex analis André. Specimens of this ant were found attacking the boll weevil by Dr. W. E. Hinds. This species is normally a honey ant, but occasionally takes insect food. It is very common in cotton fields, especially in Louisiana.

Iridomyrmex humitis Mayr. The much-feared Argentine ant has been taken attacking the boll weevil. It is, however, a friend to the weeril berallse it exterminates Solenopsis geminata, Monomorium pharaonis, and Iridomyrmex analis (Foster, 1908). 
FORMICID无.

Formicafusca (Linneus) subpolita (Mayr) perpitosa Wheeler. This species of ant is normally a honey feeder, but it is rerorded by Rangel (Rangel, 1901c) as a predator on adult boll weevils in Mexico.

Formica paltidi-fuber Latreille. A single instance of this species cutting its way into a square infested hy a bull weevil was oloserved by Mr. Hood at Ashdown, Ark., September 2, 1908.

Prenolepis imparis Say. A single instance of this species cutting its way into a square infested by a boll weevil was observed by Mr. Hood at Ashdown, Ark., September 2, 1908.

\section{BIOLOGY OF THE COHOSTS OF THE BOLI-WEEVIL PARASITES.}

The biologies of the parasites concerned in the boll-weevil complex have already been discussed. It now remains to consider the native weevils which have already or may later enter into the complex of cohosts of the boll-weevil parasites. Many of these weevils are native to the territory alrearly ocrupied by the weevil, while others will become important as new territory is added. Other families of Coleoptera and even other orders of insects may later be found to be of more or less importance as cohosts of boll-weevil parasites. The late Dr. William H. Ashmead stated that Microbracon mellitor had been reared from many Coleoptera, while Crambycobius cyaniceps bred in cerambycids and other bectles. It is important also to note the record of Cerambycobius cyaniceps from Languria. Our own observations have been confined to the Coleoptera of the families Laridæ, Anthribidæ, and Curculionidæ.

\section{PHYTOPHAGA. LARIIDE.}

(Bruchus) ${ }^{1}$ Laria salleci Sharp. This bruchid is characteristic of the Gulf Coast prairie of Texas. It hreeds in the pods of huisache (Vachellia farnesiuna), is a continuous breeder, and is wenerally highly parasitized by Crosigulphus bruchi, Corumberobius bruchicorus, Cen-

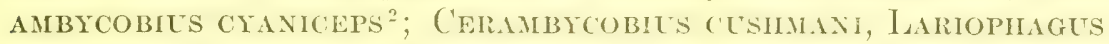
Texanus, Eurytoma tylodermatis, Horismenus sp., and several other undetermined parasites.

Laria exigua Horn. This hruchid is apparently Austroriparian and Carolinian. Its principal food plant is Amorplutiruticose, in the seed

1 The generic name Bruchus was first used by Geoffroy in 1762. Only one species is admissible in our code of nomenclature and this is Ceramby furLinnous, which is also the type of Ptinus Linnæus 1767. The genus Laria was described by Scopoli in 1703 and the type thereof has been designated as salicis Scopoli, a symonym of Dermestes pisorum (pisi) Linnæus.

Linnæus's conception of Bruchus dates from 1767 and the type thereof was designated by Latreille (1810) as Dermestes pisorum Linnæus. Hence we see that Bruchus Linnæus (1758) is preoccupied by Geoffroy (1752) and an isogenotypic synonym of Laria Scopoli (1763).

Although the genus has been subdivided into several genera, our American species have not been studied with regard to such subdivision and it is hence best to consider all as in the genus Laria, sensu latiore.

3 The names of boll-weevil parasites are printed in small capitals; others in italics. 
pods of which it breeds prolifically. It is a continuous breeder and is highly parasitized by Cerambyeobius lmevicuedus, ('ERAmbycomies CYANICEPS, IIorismenus sp., IIeterospitus prosopidis, Eurytoma sp., Microdontomerus anthonomi, Catolaccus incertus, and several other species.

Laria obtecta Say. The common bean weevil is known to be parasitized by Ceranimyobius Cynniceps and Bruchobius laticollis.

Laria compressicornis Schaeffer. This bruchid, which breeds in the pods of Acuan illinoensis, is parasitized by CERAmbycoBIUs oYANICEPS and Heterospilus prosopidis.

Laria ochracea Schaeffer. 'This bruchid, which breeds in the pods of Vicia sp., is parasitized by Cerambrcobius craniceps, C. cushMANI, Eurytoma sp., and Heterospilus prosopidis.

Spermophagus robinix Schaeffer. This bruchid is very common in the pods of the honey locust (Gleditsia triacanthos), and the water locust (Gleditsia aquatica), both of which are trees belonging to the humid Austral zones. It is parasitized by Heterospilus bruchi, CER-

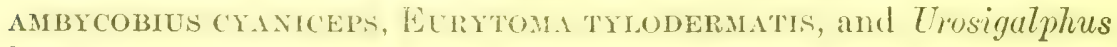
bruchi.

RHYNCHOPHORA. ANTIIRIBID X.

Brachytarsus alternatus Say. This beetle probably breeds under many different circumstances. The only records are from a fungus gall on Ipomace penduralu, and from the stems of Elymus virginicus and Sideranthus rubiginosus. It apparently belongs to the humid Austral zones. It is parasitized by Microdontomerus anthonom and a Bracon.

Arxcerus fasciculatus DeGeer. This very widely distributed Lower Austral insect (see fig. 19), known commonly as the coffee-

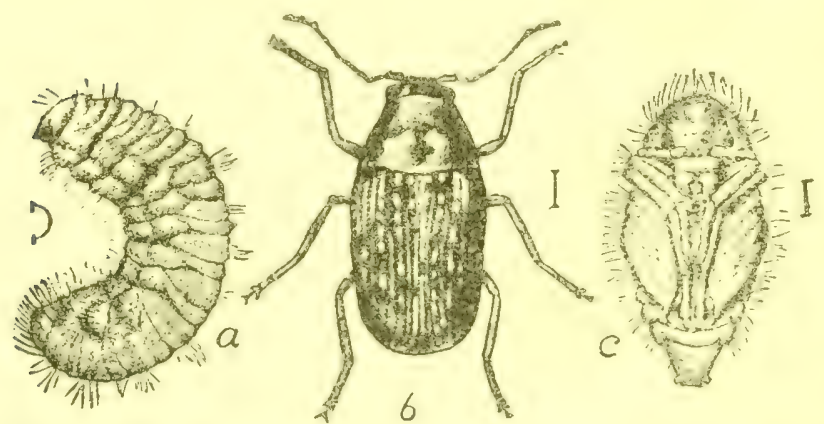

FIG, 19.-The coffee-bean weevil (Arxccrus fasciculatus), a cohost of bollweevil parasites: $a$, Larva; $b$, adult; $c$, pupa. Enlarged. (From Chittenden.) bean weevil, breeds in stored vegetable products, in the seed of Theobroma cacao, in the berry of the coffee tree (Coffea arabica), in diseased cotton bolls, in seed pods of Cassia occidentalis and $C$. obtusifotia, in seeds of Indigofere tinetoriu, in sireen and decaying fruit of Melia azedarach, in green and dry cornstalks, and in dry acarian galls on Ipomca 
lacunosa. In the Melia berries it is parasitized by Cerdurycobres cushmani, Eurytoma tylodermatis, and Pediculoides sp.

CURCULIONIDA. APIONINA.

Apion segnipes Say in Cracca virginiana is parasitized by EurrTOMA TYLODERMATIS.

Apion decoloratum Smith. Dr. Chittenden records this weevil as breeding in Meibomia paniculata and parasitized by Comonseres INCERTUS.

Apion griseum Smith. Dr. Chittenden records this weevil as breeding in Phaseolus retusus, $P$. wrightii, $P$. polystachyus, and Strophostyles pauciflore, and parasitized hy ('ATOLACCUs Ixcentes.

Apion nigrum Smith. Breeds in buds of Robinia prendacar ia and is parasitized by CATOLACCUS INCERTUS.

Apion rostrum say in pods of Baptisia is parasitized hy ('FnambCOBIUS CYANICEPS.

CLEONINÆ.

Lixus musculus Say. This weevil is known both from the Lower Sonoran and Austroriparian zones. It breeds in the stems of Poly-

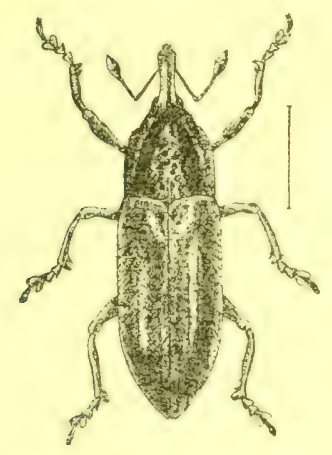

Fig. 20.-The blondweed weevil (Iticus strotichllis), a cohost of boll-weevil parasites. Enlarged. (From Hunter and Hinds.) gonum pennsylvanicum, $P$. portoricense, and $P$. punctatum, making an oblong gall or swelling. It is parasitized by EurYToMA TYlodermatis, Cerambycobius craniceps, Neocatolaceus tylodermæ, Glyptomorpha rugator, G. novitus, and Horismenus lixivoms.

Lixus scrobicollis Boheman. This weevil (fig. 20 ) is probably confined mainly to the moist Austral zones. It breeds abundantly in the stems of Ambrosia trifida, A. artemisixfolia, A. psilostachya, and Hetianthus spp. It is quite highly parasitized by Ptinobius magnificus, EURYToma tYlodermatis, CERAMBYCobius CYANICEPS, Glyptomorpha rugator, G. mavaritus, (r. Tixi, Tipio belfragci, Microdus simillimus, and Horismenus lixivorus. Mr. Townsend has described Lixophaga pare from a specimen reared from this weevil at Dallas, Tex., August 15, 1907.

\section{ERIRRHININE.}

Smirromyx tychoides LeConte. This weevil breeds in stem cralls of various species of Cuscuta. It is parasitized by Micromricon MeLLITOR and Eutrichosoma albipes. 
Desmoris scapalis LeConte. 'This weevil (fig. 21) occurs mainly on the black prairie in Texas and brecis in the heads of Sideranthus mubiginosus. It is parasitized by Microbracon Mellitor.

ANTHONOMIN.T.

Macrorhoptus sphæralcix Pierce. This weevil was found breeding in stems of Sphreralcea angustifolia. It is the host of EURYToms TYLODERMATIS.

Tachypterellus quadrigibbus Say. This fruit weevil breeds in the seed of apple, pear, Cratxgus oxyacantha, and Cratxgus mollis. It is known to us to be parasitized by Cerambycobius CYANICEPS and Catolaceus hunteri.

Smicraulax tuberculatus Pierce. This species breeds in the stems of mistletoe (Phoradendron flavescens) throughout Texas, and evidence of its work has been observed in Touisiana and Mississippi. It

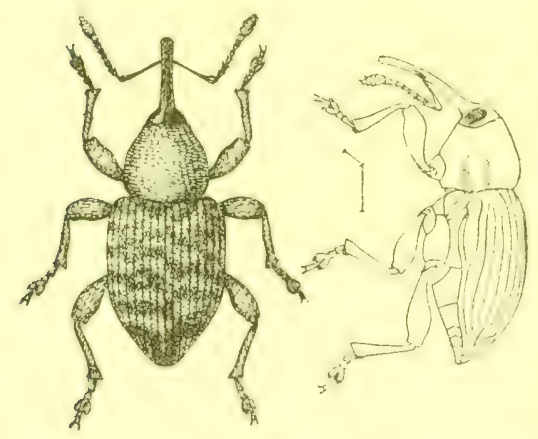

FIG.21.-The ironweed weevil (Desmoris sca. palis), a cohost of boll-weevil parasites. Enlarged. (From Hunter und Hindi.) is parasitized by Eurytoma TYloDERMATIS, CERAMBYCOBIUS CYANIceps, Catolaccus hunteri, and Microbracon melltor.

Anthonomus fulvus LeConte. This weevil breeds in the larger buds of Callirrhoe involucrata and C. digitata. It is a characteristic woodland and meadow insect in Oklahoma and'Texas. The known parasites are CATOLACCUS INCERtus and Mírobracon MELLITOR.

Anthonomus signutus Say. The strawberry weevil is mainly characteristic of the humid Austral zones, and it breeds in the buds of strawberry, blackberry, dewberry, raspberry, Rubus villosus, Potentilla canadensis, and Cercis canadensis. It is parasitized by Microbracon anthonomi, Calyptus tibiator, CATOLACCUS HUNTERI, C. INCERTus, and $C$. anthonomi. The two latter species were described from this weevil.

Anthonomus albopilosus Dietz. This little Texas weevil breeds in the capsules of Croton capitatus, C. engelmanni, and C. texense. It is known to us to be parasitized by Microbracon Mellitor, CatoLACCUS hunteri, C. incertus, and Cerambycobius CYANiceps.

Anthonomus nigrinus Boheman. 'This species is eastern in habitat and breeds in the buds of Solanum carolinense, and the potato ( $S$. tuberosum). It is the host of Entedon lithocolletidis, Eriglyptus robustus, Catolaccus incertus, and $C$. anthonomi. 
Anthonomus aneolus Dietz. This Texas weevil breeds commonly in fungus galls on the leaves and in the buts of Solanum eleagnifolium and $S$. torreyi and also in the buds of $S$. rostratum. It is parasitized by CATOLACCUS HunTERI and a Eurytoma.

Anthonomus eugenii (ano (xurotinctus Champion). The pepper weevil (fig. 2.2) breeds in most of the eultivated and wild peppers and may be considered a serious pest. It is parasitized by Catolaccus indereri, Microbricon Mellitor, and Pediculoides vextricosus.

Anthonomus squamosus leconte. This is a weevil typical of the gypsum prairie of the Lower Sonoran Zone, although occurring less abundantly in the western edge of the moist Austral zones. It breeds in the flower heats of crimdelin squarrosa muda, G. inuloides, and perhaps also on other (irindelias and Melianthi. It is known to us to be parasitized by Microbracon mellitor, Catolacous hunteri, and EuryTOMA TYLODERMATIS.

Anthonomus nebulosus LeConte. This weevil breeds in the buds of Cratregus in Louisiana and Arkansas. It is parasitized by $\mathrm{C}_{\mathrm{ATO}}$ LACCUS HUNTERI and Sigalphus sp.

Anthonomus heterothecæ Pierce. This small weevil breeds in the flower heads of Heterotheca subaxillaris and probably other asteroid flowers. It is parasitized by $\mathrm{C}_{\Delta \mathrm{TO}} \mathrm{LCCUS}$ inUNTER and EURYTOMA TYLODERMATIS. Previous recorts by the senion author on Anthonomus disjunctus LeConte all refer to this weevil.

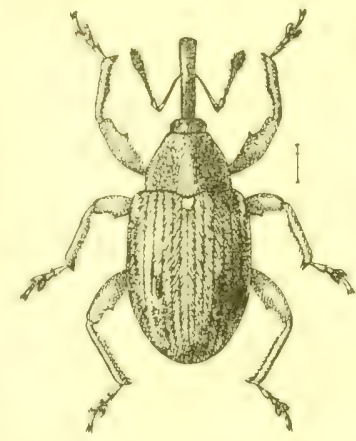

FIG. 22.-The pepper weevil (Anthrnumus curfnii), a cohost uf boll-weevil parasites. Enlatged. (From Hunter and Hinds.)

Anthonomus aphanostephi Pierce. This weevil breeds in the heads of Aphanostephus stirrobasis, and is parasitized by Catolaccus INCERTUS.

TYCHIIN.E.

Tyctius sordidus LeConte. This Austroriparian weevil breeds in the pods of Baptisia bracteata and B. leucantha. It is parasitized by Cerambycobius cyaniceps.

\section{CRYPTORIYNCHIN.E.}

Clutcodermus xue us Bohr'man, the common enwper weevil (fig. 23), is abundantly parasitized by kxwoms grobusi, and is likewise a host of Ennyomma clistoides and Sigalpirus curculionis.

Conotrachotus affinis Boheman. This weevil breeds in hickory nuts

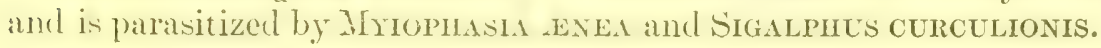


Contrachelus juglandis LeConte. This is the walnut weevil, which is also parasitized by Mropmasia ENEa, Cholomyia inæquipes, Metadexia basalis, and SigalpuUs CURCUlionis.

Conotrachetus elegans Say. This weevil breeds abundantly in the petioles of hickory, the galls of Phylloxere devastatrix on peean,
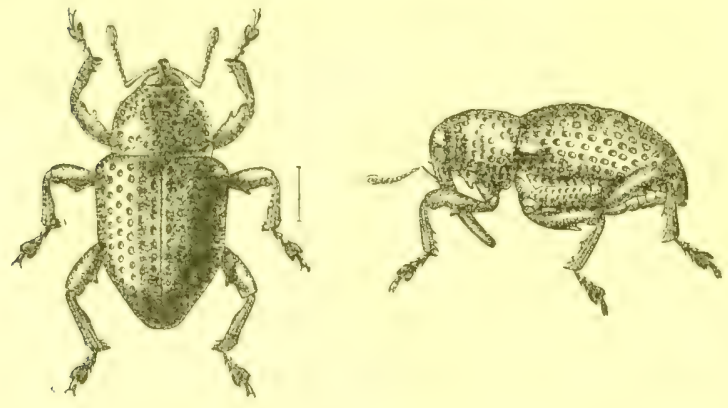

FIG. 23.-The cowpen weevil (Chalcodermus ancus), a cohost of boll-weevil parasites. Enlarged. (From Chittenden.) in pecan nuts, in leaf rolls on hickory, and finally in the roots of Amaranthus retroflexus. It is frequently parasitized by MrIOPHASIA IENEA and Sigalphus curCULIONIS, and oceasionally by Cholomyia inxquipes.

Conotrachelus nenuphar Herbst. The common plum curculio (fig. 24) breeds in the pulp of drupes and pomes. The larvæ are parasitized by Cholomyia inæquipes, SIgaLPirts cerctloxis, Microbracon yellator, and Porizon conotracheli, and the eggs by Anaphes conotracheli.

Conotrachelus naso LeConte. The common acorn weevil is parasitized by SrgalpHus CURCULIONIS.

Tyloderma foveolatum Say. This commonweevil breeds prolifically in the stems of Onagrabiennis and Epilobium. It is highly parasitized by Neocatolaccus tylodermx, Cerambycomius cyanICEPS, EURYTOMA TY-

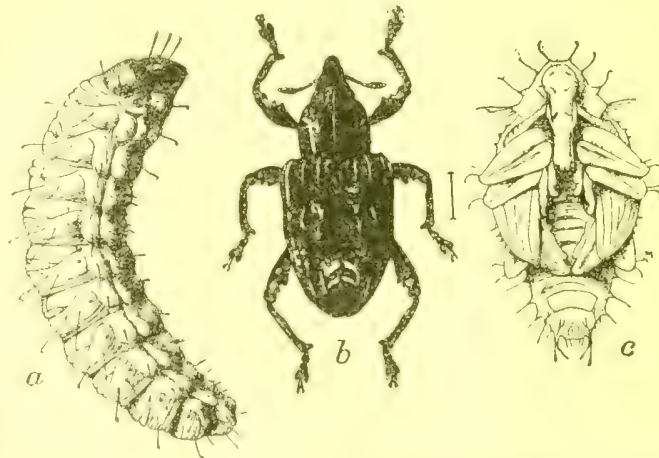

FIG. 21.-The plum curculio (Conotrachelus nenuphar), a cohost of boll-weevil parasites: $a$, Larva; $b$, adult; $c$, pupa. Much enlarged, (From Chittenden.)

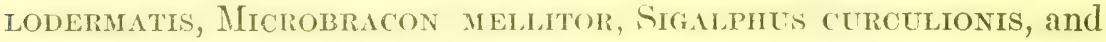
Urosigalphus sp. nov.

Gerstxckeria nobitis LeConte (Acalles). The common prickly-pear weevil is parasitized by CATOLACCUs HunterI and by several other species. 
CEUTORHYNCHINA.

Auteutes tenuipes LeConte. This weeril hreeds in the anthers of buds of Galpinsia hartuegi on the Texas black prairie at least. It is attacked by CAtolacces incer'tes, Microbracom. sp., Eutrichosoma albipes, and possibly by Catolaccus nigroxnea.

Craponius inxqualis Say. This weevil breeds in the fruit of the grape. It is parasitized by Microbracox yeldtor and Stiboscopus brooksi.

Rhinoncus myrrhopus Boheman. This weeril breeds in the stems of Polygonum and is parasitized by Cerambrconies craniceps.

Ceutorhynchus n. sp. This weeril breets in the crown of Selenia aurea and is parasitized by CATOLACCUS INCERTUS.

BARIN.E.

Baris cuneipennis Casey. This weevil breeds in the roots of Helenium temuifolium and is parasitized by CATOLACCUs inCERTUS.

Orthoris crotchii LeConte. This Lower Sonoran weevil breeds in the seed pods of Mentzelia nuda. It is parasitized very highly by Microbracon nuperus, EuRYToma tYloderMatis, and a species of Tetrastichus.

Trichobaris texana LeConte. This species breeds very abundantly in stems of Solanum rostratum, and is hence more or less a Lower Austral insect. Its parasites are CerambrcobiUs cyaniceps, Eurytoma trlodermatis, Microbracon sp., and SigalPHUS CURCULIONIS.

Trichobaris trinotata Say. The potato stalk weevil (fig. 25) breerls in the stems of many Solanacex, including Solanum carolinense, S. melongena (egg plant), S. rostratum, S. tuberosum

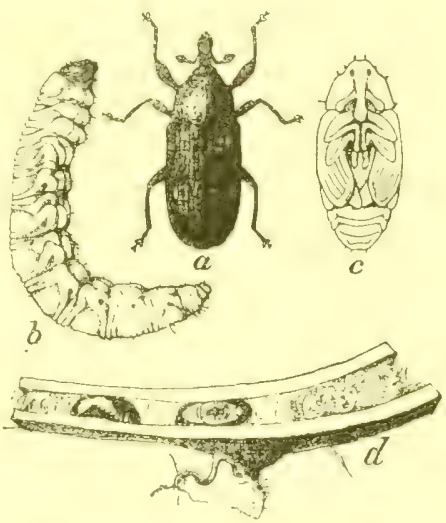

FIG. 25.-The potato-stalk weevil (Trichobaris trinotata), a cohost of boll-weevil parasites: $a$, Beetle; $b$, larra from side; $r$ pupa; $d$, stetion of putatostalk opened to show larva and pupa in situ. $a, b, c$, Five times natural size; $d$, natural size. (From Chittenden.) (potato), Datura stramonium, D. tatula, Physatis Tongifotia, P. philadelphica, $P$. Tanceotata, $P$. Theterophylla, and $P$. virginiane ambigue. It is known to be parasitized by Sigalpirus curculionis and EuRY'Toma TYLODERMATIS.

Trichobaris compacta Casey. This wecril brects in the pods of Datura stramonium and is also recorded as breeding in Datura mete-

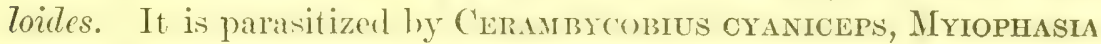
anea, and Pediculoides ventricosus. 
Ampeloglypter sesostris LeConte. The grapevine gall weevil is para-

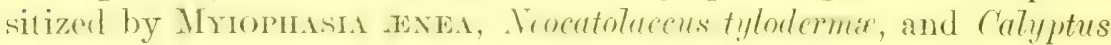
tibiator.

Zygobaris xanthoryli. Pieree. 'This weevil is abundant in the berries of Xanthoxylum clava-herculis. It is parasitized by CATOLACCUS IIUNTERI and SigALPHUS CURCUtionis.

BALANININE.

Balaninus nasicus Say. This weevil breeds in acorns. It is parasitized by Mriopiasia સENEA and possibly by Trichacis rufipes.

CALANIDINAE.

Calandra oryza Linnæus. The cosmopolitan rice and corn weevil (fig. 26) breeds in acorns of several species of oak, in galls of

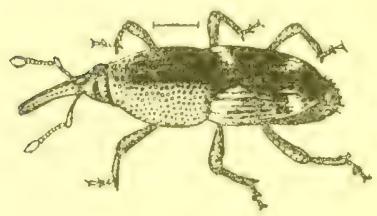

Fic: 26.-The rice weevil (Calnnitra orysu), a cuhost of boll-weevil parasites. knlarged. (From Chittenden.) Phylloxera devastatrix on Hicoria pecan, in old cotton bolls, and in all kinds of standing and stored grain. It is parasitized by Meraporus calandræ, M. vandinei, M. utibitis, M. requisitus, and Catolaccus INCERTus. Other parasites have been reported abroad.

15. $A$ LIST OF THE XIOST PLANTS OF THE COHOST WEEVILS.

In order to show more plainly the number and variety of plants whose presence around the cotton field, if infested by their typical weevils, would influence the parasite control of the boll weevil, the following list is presented, using the classification of Britton (1901):

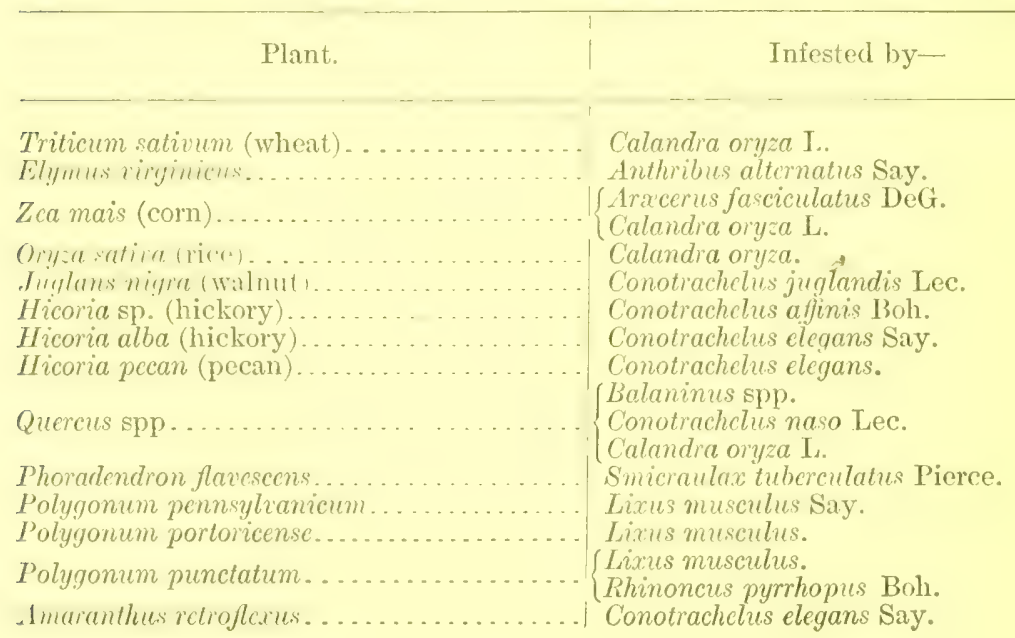




\begin{tabular}{|c|c|}
\hline Plant. & Infested by- \\
\hline 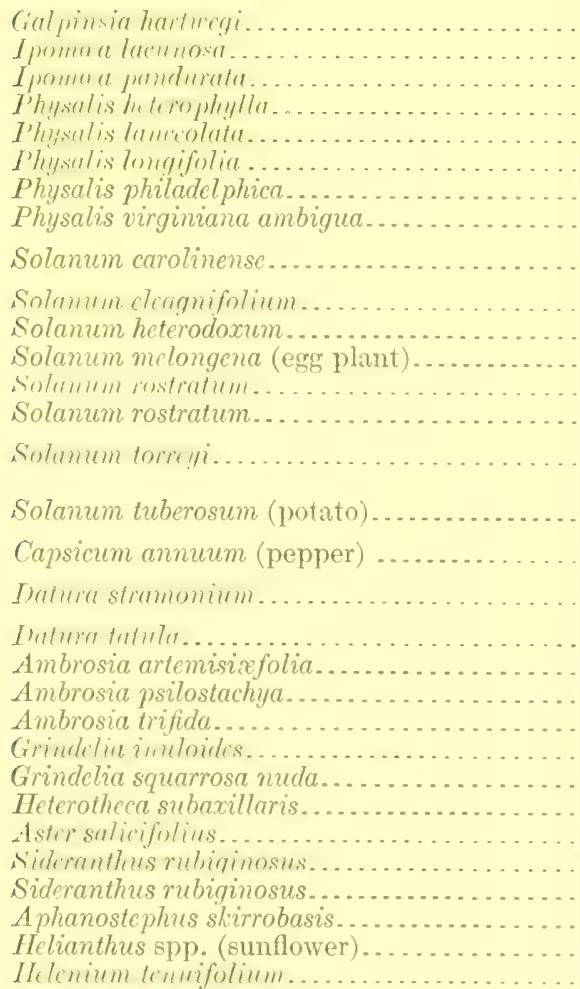 & $\begin{array}{l}\text { Auleutes tenuipes Lec. } \\
\text { Arxcerus fasciculatus DeG. } \\
\text { Brachytarsus alternatus Say. } \\
\text { Trichobaris trinotata Say. } \\
\text { Trichobaris trinotata. } \\
\text { Trichobaris trinotata. } \\
\text { Trichobaris trinotata. } \\
\text { Trichobaris trinotata. } \\
\text { Trichobaris trinotata. } \\
\text { Anthonomus nigrinus Boh. } \\
\text { Anthonomus æneolus Dietz. } \\
\text { Trichobaris texana Lec. } \\
\text { Trichobaris trinotata Say. } \\
\text { Trichobaris texana Lec. } \\
\text { Anthonomus æneolus Dietz. } \\
\text { Anthonomus æneolus. } \\
\text { Trichobaris texana Lec. } \\
\text { Anthonomus nigrinus Boh. } \\
\text { Trichobaris trinotata Say. } \\
\text { Anthonomus eugenii Cano. } \\
\text { Trichobaris trinotata Say. } \\
\text { Trichobaris compacta Casey. } \\
\text { Trichobaris trinotata Say. } \\
\text { Lixus scrobicollis Boh. } \\
\text { Lixus scrobicollis. } \\
\text { Lixus scrobicollis. } \\
\text { Anthonomus squamosus Lec. } \\
\text { Anthonomus squamosus. } \\
\text { Anthonomus heterothecx Pierce. } \\
\text { Anthonomus aphanostephi Pierce. } \\
\text { Desmoris scapalis Lec. } \\
\text { Brachytarsus alternatus Say. } \\
\text { Anthonomus aphanostephi Pierce. } \\
\text { Lixus scrobicollis Boh. } \\
\text { Baris cuneipennis Casey. }\end{array}$ \\
\hline
\end{tabular}

\section{A SUMMARY OF THE MORE IMPORTANT BIOLOGICAL FACTS.}

1. The boll weevil has 54 encmies, including parasites and predators.

2. These enemies are native to other insects which are to be found in the vicinity of cotton fields.

3. The interrelationships of the boll weevil and its parasites with surrounding insects are very complicated.

4. The parasites are sometimes found in great numbers.

5. The cotton plant, by its production of nectar, furnishes a very powerful attraction for parasites and predatory insects.

6. The development of the boll-weevil parasites is as rapid as that of the boll weevil.

7. Most of the parasite species are well distributed.

8. The parasite species attack other hosts in the spring and have a generation before the boll weevil is ready for them.

9. New species of parasites are becoming adapted to the weevil each year. 
10. Other cotton insects, by their ravages upon the food of the weevil, sometimes reduce the numbers of the boll weevil itself.

11. Much valuable work is done by the ants, which are present in many fields.

\section{PART III. THE ECONOMIC APPLICATION.}

The economic application of parasitic control to the boll-weevil problem is dependent upon accurate knowledere of a multitude of conditions. The preceding two parts of this bulletin have been deroted to a statement of the many phases of the parasite situation. It must be understood at the herinning of this part that we comsidel the utilization of parasites and other insects inimical to the boll weevil as intimately connected with good agriculture. The bollweevil problem, from a parasite standpoint, is entirely different from any other parasite problem ever studied. In other cases such means as introductions from foreign countries may be utilized. In the present case the main problem is to devise such agricultural practices as will increase the effectiveness of the parasites already present.

In order to facilitate the treatment of the economic methods to be sugrested, this part is also divided into seretions. which are as follows:

1. The economic principles inrolved.

2. Interpretation of parasite statistics.

3. Interpretation of the biological complex.

4. How to profit by existing conditions.

5. How to plan for the greatest possible control.

6. Propagation and artificial introductions.

7. Objectionable practices.

8. The economic significance of the investigation.

\section{THE ECONOMIC PRINCIPLES INVOLVED.}

The attempt at utilization of insect enemies in economic entomology is now receiving so much attention that the authors will set down the principles which appear to have been the foundation of the work they have done.

1. Insects in a state of nature are more or less completely hold in check by natural acencies, in which of her inserts frequently figure as of direct or indirect importance. Many insects are controlled almost entirely by their insect enemies. No insect is without its natural checks.

2. The relationships between an insect and its cnemies can not be expressed by a simple ratio, nor are they in any way invariable. The agencies operating for and against the welfare of a giren species are so many and of such inconstant magnitude, due to the activities 
of other agencies, that the effects of one or two agencies of control with known strength can not be estimated, because of the many other agencies either unknown or of unknown strength.

3. When an injurious insect escapes from its natural surroundings to a region where anditions are farorable for enormous reproduction, it may become a pest, but it is never absolutely free of natural checks. Anthonomus grandis has never been free of its checks although it escaped those of its native home. These agencies of insect control are inherent to all countries: An insect parasite is as likely to escape its original surroundings as a phytophagous insect.

4. When an insect finds in its vicinity a variety of food closely related to its native host, and that food is more succulent or more abundant, there is a possibility that sooner or later the more inviting food will berome the normal food. This possibility becomes stronger when the original food supply fails, if the species is to be continued. Not only phytopharous but antomophagous insects have frequently been proven to have thus changed their food habits-whether from preference or necessity it is not known. Leptinotarsa decemlineata (the Colorado potato beetle) is an excellent example of this change of habit among phytophagous insects. All of the boll-weevil parasites are examples of parasites which have adjusted their habits in the presence of their original hosts.

5. A crisis in the history of a species occurs whenever the food supply fails. The species may either disperse in search of food, as the boll weevil does each autumn, or it may hibernate or restivate, or it may select a new host, or the species may perish. All these results occur in nature. All of these alternatives may be chosen by different individuals of the same species. It is safe to assume that when a species is found to have many hosts that it has undergone many crises and that the resultant species is a highly developed and adaptable form. A species most limited in food habit is most liable to restriction or extinction and consequently of a lower type than one able to meet any emergency.

6. When a desirable parasite species is known to be adaptable to various hosts, a crisis may be artificially superinduced by the timely elimination of the favorite hosts, thus forcing the species to attack the most predominant near-by related host in tho vicinity, or it may be taken to an entirely remote or foreign locality and placed near a field containing many insects closely related to its original host, which it may learn to thrive upon.

7. The species most available for utilization are those most adaptable to changing environments, or those having the most hosts in the given locality. These other hosts will serve as nurseries for parasites. 
8. Certain parasites with more or less established habits require that their hosts be in certain habitual locations (for example, Neoratorlaccus requires stem-dwelling hosts), and in like manner there are conditions which can be made more favorable for parasite attack through cultivation or through plant selection. Furthermore, since parasites require different conditions, it is desirable to alter the existing conditions so as to malie them farorable for as many species as possible. In the case of insects extending their range over many different climates it should be the aim to introduce parasites best adapted to the prevalent conditions.

\section{INTERPRETATION OF PARASITE STATISTICS.}

From the great mass of parasite statistics given in Part I a number of important facts need to be considered.

Parasitic and predatory attiuk is strongest from Lugust until frost time. Hence it may be presumed that whatever artificial propagation is to be done will be most profitable when condureted during this period, provided it does not interfere with early fall destruction of stalks, the fundamental cultural remedy against the boll weevil.

The greatest control of the boll weevil by insects and also by all agencies is in hanging squares. As has been stated in Part I (sec. 3), the hanging squares are at result of a diagonal absciss layer, which causes the drying square to fail in separating itcelf' ('ompletely from the plant. These squares die on the plant and aflord a very favorable position for parasitic attacks upon the weevils within. The statisties show that insert control in fallen squares is greatest in the moist States of Lomisiana and Mississippi. This is undouldedly due to some of the new parasites which are accustomed to attacking woodland weevils and other insects characteristic of this humid region. The insect control in hanging squares is the greatest in the comparatively dry States of Texis and Oklahomia. These dry States also have a higher combined natural control in the fallen squares than in the hanging squares, larrely becallse the climatic conditions cause a higher mortality of weevil stages in squares lying on the heated surface of the ground. On the contrary, the humid States have a higher mortality from both climatic and insect agencies in hanging squares than in fallen. Furthermore, it has been proven, in Part I (sec. 4), that an increase in the amount of hanging squares will increase the total control. Having these facts in mind, the obvious conclusion is that it will be desirable to have varieties of cotton which have this tendency best developed. Among the varieties which are now known to retain their squares are the cluster varieties, including the Rublee. 
The figures show that parasitism becomes very high under favorable conditions and also that agrieulture modifies the insect control. Obviously therefore those anricultural methods which will favor the highest insect control must be sought. These methods, as now known, will be dealt with more fully in a following section.

It was feared for a long time that the parasites of the weevil woukd be held in control by the warm climatic conditions which affect the boll weevil. This is not so. We have found abundant proofs of the facet that a temperature which will kill the boll-weevil larval will not kill the errer or small parasite larva in the same cell, and that the parasites can develop to maturity on the dried remains of the weevil. The temperature fatal to the boll weevil is $123^{\circ} \mathrm{F}$., at temperature frequently reached on a hot burning soil. We have found in several years that a low temperature which will kill the boll-weevil larva is also not fatid to the prarisites, for in November, 1907, when 97 per cent of all the boll-weevil stages were frozen, no evidence whatever could be found of mortality among the parasites. The minimum fatal temperature of the boll weevil is $12^{\circ} \mathrm{F}$.

\section{INTERPRETATION OF THE BIOLOGICAL COMPLEX.}

The compliwated hiologiral factors which have been noted in Part II have been summarized briefly in section 16 of that part (p. 82). The interpretution of these facts has been suggested in an number of places throughout, the second part. Hence only a few words are necessary at this point.

The finct that surrounding each cotton field there are numerous plants harboring weevils and their parasites is of extreme importance in this problem. 'Theso parasites are generally capable of attarking the boll weevil under conditions of necessity or alternative choice. The aim is therefore to find all the methods by which these parasites may be forced to leave their native hosts and attack the boll weevil. In fact, the entire second part has been devoted to giving these facts in order to bring out this single point.

\section{HOW TO PROFIT BY EXISTING CONDITIONS.}

COLLECTION OF COTTON SQUARES IN SCREENED CAGES.

As has just been pointed out, there are conditions around the cotton fields which are potential of a considerable increase in the parasitic control of the boll weevil. Probably no other method will yield better results than the gathering of the cotton squares which are infested and placing them in wire-screen cages of 16 or 18 mesh to the inch and placing these cages in selected parts of the cotton fields. This method is not new in entomological practice. It has 
been used with great value in the freeing of apple orchards in Europe from the apple-bud weevil (Anthonomus pomorum). The boll weevils can not pass through a 16-mesh or 18-mesh wire screen, while the parasites can do so, and therefore the release of these enemics will be constantly increasing the proportion of parasites against the weevils. Even if a 14-mesh wire screen is used, only at small proportion of the weevils can escape through it and some gain is eflected by the release of the pariasites. In order to demonstrate numerically just how this would happen, three hypotheses are presented. In the first case squares are collected and put in a 16-mesh wire cage, and in the second calse squares are collected and put in a 14-mesh wire cage, and in the third case no squares are collected. 'The averinge percentage of control which follows as a result easily demonstrates the advantage which will be almost immediately gained.

It has been contended and proven that many weevils escape through the ordinary wire screen.

\section{I.-Given 10,000 developing stages in a 1-acre field.}

(A) Collect squares containing 50 per cent of the stages and place in 16-mesh

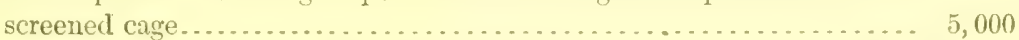

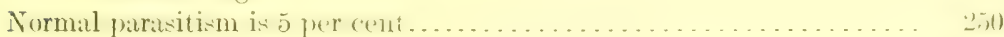

Ants and heat kill 40 per cent............................... 2, 000

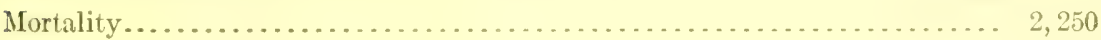

Breed............................................... 2,750

There escape through 16-mesh screen-

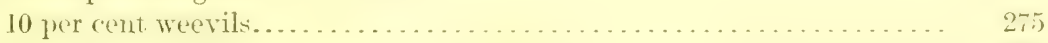

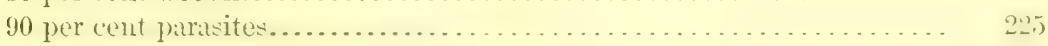

(B) There remain in the field squares containing 50 per cent.......... 5,000

Normal mortality as above, 45 per cent ..................... 2, 250

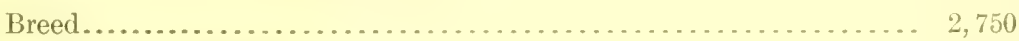

Some weevils escaped from cages............................. 275

Total weevils at end of generation....................... 3, 025

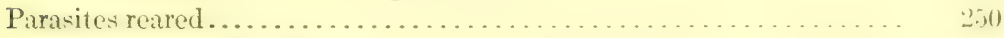

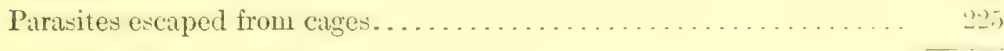

Parasites present in field.............................

There is I parasite to every 6.3 weevils.

\section{II.-Given conditions as above, squares in 14-mesh screened cage.}

(A) From collected squares breed.

There escape through 14-mesh screen-

40 per cent weevils.................................. 1, 100

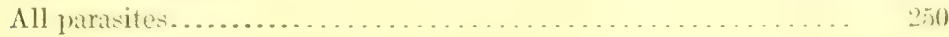

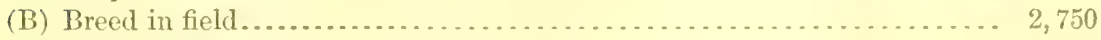

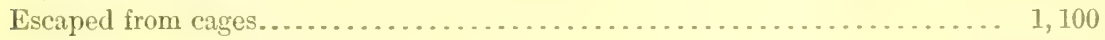

Total weevils at end of generation...................... 3,850 
Parasites reared.................................... $\quad 250$

Parasites escaped.................................. 250

Parasites in field................................. 500

There is 1 parasite to every 7.7 weevils.

III.-Given 10,000 developing stages in a 1-acre field.

No squares are collected................................. 10, 000

Normal parasitism, 5 per cent.......................... 500

Ants and heat kill 40 per cent............................. 4,000

Mortality...................................... 4,500

Breed.............................................. 5,500

Parasites reared, 500 .

There is 1 parasite to every $1 \mathrm{~L}$. weevils.

SUMMARY.

\begin{tabular}{|c|c|c|c|}
\hline & \multirow{2}{*}{$\begin{array}{c}\text { Squares not } \\
\text { collected. }\end{array}$} & \multicolumn{2}{|c|}{$\begin{array}{l}\text { Squares collected and } \\
\text { placed in- }\end{array}$} \\
\hline & & $\begin{array}{l}\text { 14-mesh } \\
\text { wire cage. }\end{array}$ & $\begin{array}{l}\text { 16-mesh } \\
\text { wire cage. }\end{array}$ \\
\hline 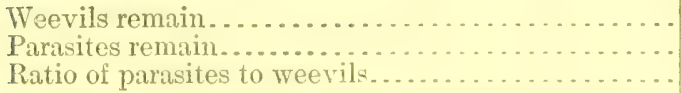 & $\begin{array}{r}5,500 \\
500 \\
1: 11\end{array}$ & $\begin{array}{r}3,850 \\
500 \\
1: 7.7\end{array}$ & $\begin{array}{r}3,025 \\
475 \\
1: 6.3\end{array}$ \\
\hline
\end{tabular}

ELMINATION OF COHOSTS.

Another practice of undoubted value in bringing about a higher percentage of patasitisin upon the boll weovil is the elimination of the cohosts of the boll-weevil parasites at proper times. To show what has been done in this line, the case of the Dallas farm in 1907 may he cited. On July 19 of that yoar a rery largo hedge of weeds, Ambrosia trifuda, infosted hy Lixus scrobicollis was cut. Theso weeds wero along the fonce aljoining a part of the cotton field which had boen under close observation for parasites throughout 1906 and the spring of 1907. In 1906 there was not found in any plat on this farm a higher parasitism than 2 per cont by Eurytoma tylodermatis in hanging squares. Earytoma was very numerous in the Ambrosia weeds next to this field, but did not appear to attack the weevil in large numbers. Before tho weeds wero cut in 1907 the two plats nearest these weods averaged 26.76 por cent and 16.79 per cent parasitism by Eurtoma. On August 17, about a month after these weods were cut, the two plats just mentioned had, respoctively, 37.50 per cent and 26.66 per cent parasitism by Eurytoma. This striking grain aljoining the weeds was not reflected by parts of the field farther removed. 
EARLY DESTRUCTION OF THE COTTON STALKS.

There can be little doubt that the early destruction of the cotton stalks, in addition to depriving the boll weevil of its food plant, will also cause the parasites to seek a series of hosts which can carry them through the winter period. In order to prove that fall destruction does not have an injurious effect upon parasite control, we would cite the discussion of the Victoria fields, in which various methods of fall destruction were carried out, as discussed in Part I, section 6. As a further proof tho famous Olivia fall-destruction experiments may be considered.

On October 1 to 10, 1906, all of the cotton plants in over 400 acres, constituting the entire Olivia cotton community in Calhoun County, Tex., were cut and burned under the direction of Mr. J. D. Mitchell. According to the rearing records in our possession, the parasites developing in this cotton would all be mature before November 10, and if they hibernated, would have to do so as adults. Noother cotton existed within 12 miles, as the community is completely isolated by water and marshland.

Cotton was planted about March 15, 1907. On $\Lambda$ pril 15 no bollweevil work could be found, but on May 7 a single weevil was found after a careful examination of eight fields. On the same date at Six Mile settlement, across the bay near Port Lavaca, there was considerable infestation. If the parasites hibernated as adults they would be dead long before the middle of June. If they could have hibernated as immature stages they would have matured by Narch 15, and under normal conditions three generations would have passed by June 15. The infestation was still very slight in July. It must be argued, therefore, that any boll-weevil parasites must be breeding on some other weevil, if they did not perish.

On August 22, 1907, Mr. Mitchell found parasites with weevilinfested squares on a field in the opposite part of the community to that in which he first found the weevil infestation. The obvious inference is that a rotation of hosts occurred during the period of the boll weeril's absence.

Having planned the cropping system, it is also best to prepare the fields early for cotton and plant as early as possible. Of course, most of the reasons for early planting of cotton are well known and the practice is very common, but in this connection it must be said that such early planting has the actual advantage of enabling the parasites to start early.

Care must be given to the choice of the cotton variety which is to be used. Frequent recommendations have been made of varisties with light foliage, early maturing fruit; short nodes, and determinate growth. All of these qualities are favorable to parasite control, 
especially since such plants afford much more sunlight on the ground. The ants and also the parasites prefer much more to attack the squares which are dried out than moist squares. It seems that they can more readily penetale the linings of the square. In addition to these qualities of the cotton variety, the use of a variety with at least a moderate amount of nectar is also advised. The reason for this has been explained in preceding paragraphs. Finally, the tendency of plants to retain the squares must be again mentioned. If a variety can lumish the desired qualities of early producing, productiveness, and guality of lint, as well as a diagonal ahsciss layer on the square, that variety should be chosen above others.

If at all possible, it is advisable to plant the rows far apart or on the check-row system, in order to give the necessary amount of sunlight. The cultivations to follow this should be with the purpose of obtaining a dust mulch, for with such a mulch the surface of the soil may be heated to a much higher degree than by deep and lumpy cultivation, and the control of the boll weeril will thus be greatly increased, through the drying effect upon fallen squares.

\section{HOW TO PLAN FOR TIE GREATEST POSSIBLE CONTROL.}

As it has been proven that many agricultural processes are favorable to the development and attack of parasites and enemies, there can be no question but that it is desirable to plan to obtain the greatest amount of this beneficial aid.

There are a few plants which have no objectionable qualities in themselves which might with good reason he planted adjacent to the cotton fields in order to induce the attack of weevils which act as hosts of the boll-weevil parasites. For instance, the presence of a hedge of blackberries or dewberries along the fence means the presence of Anthonomus signatus, the blackberry bud weevil, with its numerous parasites, all of which attack the boll weevil. The parasites would be able to carry on a generation in the spring before the boll weevils were breeding and would mature in plenty of time to attack the first developing stages of the boll weevils.

It would seem advisable to plant a hedge of the flowering shrub Amorpha fruticosa, which is the host plant of Laria exigua. This little wevil is very abundantly parasitized.

In planning the cropping system there can be no possible harm in arranging to have a forage or hay crop adjacent to the cotton field. In case a forage crop is used, cowpeas with the ever-present cowpea pod weevil would undoubtedly bring about the presence of several important parasites. The early removal of the cowpeas for fodder would force the parasites to attack the boll weevil. In the case of a hay field, the process of haying and subsequent curing 
would enable the parasites present in the various weeds to escape and attack the most abundant host, namely, the boll weevil.

If, with all these precautions, the boll weevils are very numerous in the field, and the expense is not too great, much can be gained by picking the squares and plaxing them in cagen, ats hats been described in a previous section.

Finally, at the proper season for haying, the actual methods of cutting and preparing the hay will without doubt furnish still wreater control to the weovil. Some time in september, if not before, whet her haying is carried on or not, there should be a thorough cutting of all weeds around the cotton field in order to force the parasites to the boll weevil and also to get rid of favorable hibernation quarters for the boll weevil.

\section{PROPAGATION AND ARTIFICIAL INTRODUCTIONS.}

The propagation of parasites under artificial conditions and their introduction are attemded with a great amount of labor and expense and have many technical diffieulties. The simplest form of propagation is the collection of infested squares at one locality and the shipment of them to another locality, where they are placed in the fied to await results. There are good reasons for attempting thus to introduce parasites. It has been found by very close observations that the parasites are not evenly distributed, but that each species has a more or less definitely defined geographical region. This is no doubt due to the distribution of the normal host weevils. The purposes of introduction are to take these parasites from their native localities and place them in geographical regions in which they do not at present exist. Definite proofs that results can be obtained in this manner were to he had at Dallas on the experimental farm in 1906 and also in 1907 . The 1906 experiment has been fully described in the first report on the parasites of the boll weevil (Pierce, 1905a). In 1907 a similar experiment was tried hy the release of laree numbers of adult parasites. These parasites were carried to a field in small screen cages cont aining foliage, so that the parasites might not become overheated. The cages were opened in the shade, and the parasites allowed to fly out in any direction which they pleased. While many species of parasites were released in this manner, they did not all show the results that were expected, but the release of Catolaccus incertus in a given part of the field accomplished an increase in the control in hanging squares by this species. In two of her parts of the field Microbracon mullitor was released, and it also showed crond gains. As Microbracon was released on this farm both in 1906 and 1907, it may be useful to comprare the prereentages of parasitism at rarious periouls. In August, 1906, this species furnished 8.5 per cent parasitism in hang- 
ing squares; in September, 1906, this had risen to 10.2 per cent; in July, 1907, the parasitism by this species was 35.2 per cent, and in August, 1907, it had risen to 39.8 per cent.

At Shreveport, La., in 1908, many specimens of Catolaccus incertus and Microbracon mullitor were reteased. Tiable XXIT gives an idea of the results and shows the expected increase by Catolaccus in both hanging and fallem squares and by Microbracon in hanging squares.

TABLE XXII.-Experiment in artificial introduction of Catoloccus incertus and Microbracon mellitor', Shreveport, La., 1908.

\begin{tabular}{|c|c|c|c|c|c|c|c|c|c|}
\hline \multirow[b]{2}{*}{ Class of forms. } & \multirow[b]{2}{*}{ Plat. } & \multirow[b]{2}{*}{ Date. } & \multicolumn{4}{|c|}{ Percentage of mortality. } & \multicolumn{3}{|c|}{ Gain in mortality. } \\
\hline & & & Total. & $\begin{array}{l}\text { Para- } \\
\text { sites. }\end{array}$ & $\begin{array}{l}\text { Cato- } \\
\text { laccus. }\end{array}$ & $\begin{array}{l}\text { Micro- } \\
\text { bracon. }\end{array}$ & $\begin{array}{l}\text { Total } \\
\text { paria- } \\
\text { sites. }\end{array}$ & $\begin{array}{l}\text { Cato- } \\
\text { laceus. }\end{array}$ & $\begin{array}{l}\text { Micro- } \\
\text { bracon. }\end{array}$ \\
\hline $\begin{array}{r}\text { Fallen squares... } \\
\text { Do........... } \\
\text { Do } \ldots . . . \\
\text { Do............ } \\
\text { Hanging squares. } \\
\text { Do.......... }\end{array}$ & 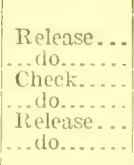 & $\begin{array}{lr}\text { Oct. } & 5 \\
\text { Oct. } & 28 \\
\text { Oct. } & 5 \\
\text { Oct. } 28 \\
\text { Oct. } 5 \\
\text { Oct. } 29\end{array}$ & $\begin{array}{l}36.44 \\
37.96 \\
40.00 \\
42.33 \\
47.15 \\
64.86\end{array}$ & $\begin{array}{r}5.93 \\
15.74 \\
10.50 \\
16.00 \\
9.90 \\
37.84\end{array}$ & $\begin{array}{r}4.23 \\
10.80 \\
5.55 \\
8.00 \\
4.39 \\
10.81\end{array}$ & $\begin{array}{r}1.70 \\
2.16 \\
3.70 \\
5.00 \\
3.29 \\
18.91\end{array}$ & $\mid \begin{array}{r}\text { Per cent. } \\
165 \\
\cdots \\
\cdots \\
252\end{array}$ & $\mid \begin{array}{r}\text { Per cent. } \\
150 \\
\cdots \\
\cdots \\
148\end{array}$ & $\begin{array}{r}\text { Per cent. } \\
33 \\
335 \\
377\end{array}$ \\
\hline
\end{tabular}

In order to obtain satisfactory results from the release of infested material, it is necessary to place the material in cages from which the injurious weevils can not escape but which will still allow the parasites egress. This principle has been explained in other sections. There is also another important consideration in the construction of the cages. When a large amount of material such as this is collected in a small space it furnishes great inducements to attack by colonies of ants. The only way that the material can be protected from total destruction by ants is the isolation of the cage on legs by the use of "inverted cups" containing oil, or by greasing the legs in some manner.

\section{TRANSFER OF ANT COIONIES.}

Since the work of ants is always very favorable to control, means should be devised of increasing their numbers in the cotton field. The dust-mulch method of cultivation is very favorable to the ants in that it does not disturb their colonies after they have commenced breeding. This is a very important matter to consider. The late Mr. F. C. Pratt, in working with the horn fly (Lyperosia irritans L.) discovered that fresh manure containing numerous fly larvæ is very attractive to Solenopsis, and that these ants seem to transfer their whole colony at times to the manure. Mr. Wilmon Newell, in connection with the Argentine ant investigations, at a later date, found that he could trap immense colonies of the Argentine 
ant (Iridomyrmex humitis) by means of boxes containing manure. These observations are very suggestive, for they point out the possibility that colonies of ants can be obtained by placing fresh manure in boxes near ant colonies. When sufficient numbers have entered, they may be boxed up for removal to a place desired. In this manner great colonies could be transfered bolily for comsiderable distances.

\section{OB.JECTIONABLE PRACTICES.}

There are several practices which are quite objectionable from the standpoint of encouraging the parasites and most of which have also been found objectionable from purely cultural standpoints. When the cotton is planted closely on moist soil its growth is mainly vegetative and consequently immense stalks may have very little fruit. Agriculturists have always pointed out that large cotton plants need plenty of room in order to produce fruit. Field examinations to determine the mortality of the boll weevil from various causes have always shown that the parasitism is greatest in the portions of a field where the foliage is lightest. A notable example was found at Natchez, Miss., where in a single field the growth was very irregular. One spot seems to have been used for feeding cattle and was very fertile. On this spot the cotton grew 6 or 8 feet tall and the grounc? was densely shaded. Here the mortality of the weevil was very low, and there was scarcely any control by insects. One hundred feet from this was a thin piece of ground where the cotton plants were barely 2 feet high, but they were loaded with bolls and showed a much higher percentage of mortality, especially by insect enemies. An actual count of the mumber of bolls in the two parts of the field was greatly in favor of the smaller plants.

Late planting has been proven objectionable from almost every standpoint from which it has been viewer. Under existing circumstances there are no valid arguments for late planting. From the standpoint of control hy parasites late planting simply delays the attack of parasitic (enemies and restueses the amount of control in the fall at a critical timo.

It is believed that the use of varieties which always tend to drop their squares is objoctionahlo if rariotios with the opposite tendeney can be found with the same qualities of production.

The practice of picking squares and then burning them can not be condemned too strongly. The planters are by this practice almost nullifying the good work that ther to hy piching the squares. They are doing nothing more or less than destroying their best friends when they burn these squares. This may be proven by an hypothesis

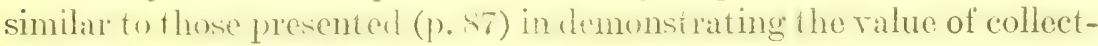
ing the infested squares. 
Given 10,000 developing stages of the boll weevil in a 1-acre field.

(A) Collect and burn squares containing 50 per cent of the stages..........5, 000

This destroys all parasites as well as weevils.

(B) There remain in the field squares containing 50 per cent of the stages

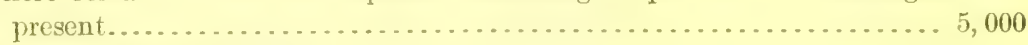

Normal parasitism 5 per cent............................. 250

Ants and heat kill 40 per cent.............................. 2,000

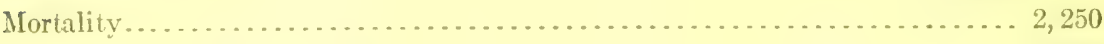

Weevils to breed ................................... 2,750

Parasites present in field, 250.

There is 1 parasite to every 11 weevils.

This method undoubtedly greatly reduces the total number of weevils in the field, but, as can be readily seen, the proportion of parasites to weevils is the same as if nothing had been done-namely, 1 to 11 . It was shown that by placing the squares in 16-mesh wire cages the proportion would be 1 to 6.3. Hence it appears that by the caging method the planter leaves in his field a more active agency than he had before to attack the many weevil stages he has undoubt. edly missed, whereas by the burning method he has not in the least improved his field conditions.

\section{S. THE ECONOMIC SIGNIFICANCE OF THE INVESTIGATION.}

In final summary the following points are emphasized:

I. The control of the boll weevil by insect cnemies is sufficiently great to give it a high rank in the struggle against the pest. A considerable portion of the insect control would not be accomplished by any other factor; hence it is by no means to be neglected.

The number of species of insects attacking the developing stages is 49 .

The control in any given place consists of the combined work of several different species.

Places having the largest number of controlling insects have the highest percentage of control.

In many places insect control is considerably greater than climatic control or than any other class of factors.

The average insect control is 20 per cent of all immature stages or two-fifths of the entire natural control.

The cotton leaf-worm is a valuable enemy of the boll weevil when it defoliates the cotton after September 1, a date beyond which new squares can not be expected to mature. It kills many weevils by starvation, kills many others while consuming the squares, and finally forees a premature hibernation which is generally fatal. 
II. The amount of control due to the various factors at work in any given place should be increased if possible. Parasites can be introduced into new fields.

In order to prevent serious injury to cotton, the mortality of the weevil should he above 90 per cent. It has averaged over 57 per cent for four years and has reached almost 100 per cent several times.

While climatic influences orcasionally luing the control above 90 per cent, they can not be regulated or in any way directly utilized.

Although the insect enemies present at a given place may be accomplishing the greatest amount of work possible, the fact remains that if other species of enemies are introduced and become established the control can be increased.

Since certain parasites attack the weril preferably in dry locations and others in wet locations, and since some prefer to attack the infested forms on the plant, while others seek them on the ground, it is possible to select the insect agencies so as to obtain the least amount of lost or duplicated energy.

III. The parasites and predators which attack the boll weevil are native insects, already present in a given territory before the weevil arrives.

The predators, especially ants, will attack almost any lind of insect food. The parasites in nearly all cases are capable of attacking almost any kind of weevil breeding in herbs above ground or in fruits.

The parasites have proven their ability to adjust themselves to the boll weevil by attacking it in its first generation in newly infested territory in instances where the actual sources of the parasites were demonstrable.

The weeds surrounding the cotton fields contain many weevils which are harboring multitudes of arailable parasites. These parasites may be induced to attack the boll weeril by the timely elimination of their native hosts.

This leads to the recommendution that planters cut the weeds adjoining the cotton fields, along the roadsides, turn rows, and fences about the time of the maturing of the crop.

It also teads to the recommendation that a ficld adjoining the cotton be used as a pasture or hay field, and that this. ficld be moved early in the fall. 'The usual haying will also bring about the same result namely, the elimination of other plants harboring weevils which attract the parasites needed in the cotton patch.

It is advisable to have in the vicinity of the cotton field such plants as the dewherry, Croton, Amorphil, cowpeas, ete., which contain other hosts of the boll-weevil parasites. 
IV. The cultural methods of controlling the cotton boll weevil are the most favorable methods of cotton culture from the parasitic standpoint.

The tendency of the principal boll-weevil parasites to prefer light and heat leads immediately to a long series of recommendations.

Heat on the ground is essential for the climatic control of the weevil and also for parasitic control. Although the two factors overlap, each accomplishes considerable independent control.

A heated condition of the ground may be accomplished by planting the rows far apart, by check-row planting, by flat cultivation, or by planting varieties which have short limbs, or shed their foliage early, or have small leaves.

Fall destruction of the cotton plants cuts off the parasites from breeding on the weevil but sends them to other hosts upon which they can breed a winter generation, or pass hibernation, thus gaining not only a generation on the weevil but providing for themselves during the winter.

The early planting of cotton in the spring makes it possible for the earliest parasites to attack the weevil.

$V$. The tendency to retain infested fruit, which is displayed by certain varieties, is worth consideration.

The fuct that many more parasites are reared in hanging squares than in fallen squares makes it desirable in humid regions to have many of the hanging squares in a field in order to serve as a nursery of parasites for the weevits in the fallen squares.

Varieties which show an elongate diagonal connection between the square and stem will tend to have an imperfect absciss layer formed. Prominent in this class are the chuster boll varieties of cotton. It is recommended that search be made for such a variely as will retain its infested forms and at the same time fulfill the other requirements in the mating of a good crop.

VI. Any step which will diminish the number of weevils and not diminish the number of parasites in a field will of course increase the percentage of parasites present.

The most important step of this kind is the collection of infested squares and placing them in cages with a screen through which the weevils can not escape but the parasites can.

Ant colonies may be introduced into the fields in boxes of fresh manure.

If 'squares are collected, they should not be burned, but should be placed in screened cages. 


\section{BIBLIOGRAPHY.}

ALDRICH, John MERTON.

1905. Catalogue of North American Diptera. <Smithsonian Misc. Coll., vol. 46, no. $1444, \mathrm{pp} .426,427$.

Ashmead, William Harris.

1896. Descriptions of new parasitic Hymenoptera, 11. <Trans. Amer. Ent. Soc., vol. 23, pp. 218-219.

1902a. A new Bruchophagus from Mexico. < Boletin de la Comision de Parasitologia, vol. 1, no. 9, pp. 404. (For Spanish translation see Herrera.)

Bants, Nathan.

1904. A treatise on the Acarina or mites. <Proc. U. S. Nat. Mus., vol. 28, no. 1382, pp. 74-76.

1906. A revision of the Tyroglyphidæ of the United States. <U.S. Dept. Agr., Bur. Ent., Tech. Ser. 13, p. 17, Pl. IV.

BarReda, L. DE LA.

1904. El picudo del algodón. Salvacion de la riqueza de la frontera. <Comision de Parasitologia Agricola, Cir. 6, pp. 14-27.

Chittenden, Frank Hurlbut.

1893a. The strawberry weevil. <U. S. Dept. Agr., Div. Ent., Ins. Life, vol. 5, pp. 181-182.

1893b. Observations on some hymenopterous parasites of Coleoptera. <U. S. Dept. Agr., Div. Ent., Ins. Life, vol. 5, p. 250.

1895. The potato-bud weevil (Anthonomus nigrinus Boh.). <U. S. Dept. Agr., Div. Ent., Ins. Life, vol. 7, pp. 351-352.

1897. The strawberry weevil (Anthonomus signatus Say). <U. S. Dept. Agr., Div. Ent., Cir. 21, p. 4.

1899. Insects injurious to beans and peas. <U. S. Dept. Agr., Yearbook, 1898, pp. $242,245$.

1902. The potato stalk weevil, <U.S. Dept. Agr., Div. Ent., Bul. 33, n. s., p. 17.

1908. An injurious North American species of Apion, with notes on related forms. <U. S. Dept. Agr., Bur. Ent., Bul. 64, Pt. IV, pp. 30-32.

Cook, Orator Fuller.

1904a. An ant enemy of the cotton boll weevil. <U.S. Dept. Agr., report 78, pp.7, May 27.

1904b. Report on the habits of the kelep, or Guatemalan cotton-boll weevil ant. <U. S. Dept. Agr., Bur. Ent., Bul. 49, 15 pp., August.

1905. The social organization and breeding habits of the cotton-protecting kelep of Guatemala. <U. S. Dept. Agr., Bur. Ent., Tech. Ser. 10, pp. 55.

Coquillett, Daniel William.

1897. Revision of the Tachinidæ of America north of Mexico, a family of parasitic two-winged insects. <U. S. Dept. Agr., Div. Ent., Tech. Ser. 7, pp. 50,51 .

Crawford, James Ceimberlain.

1907a. New hymenopterous parasites of Anthonomus grandis Boh. <Can. Ent, vol. 39, pp. 133, 134, April.

1907b. New North American Hymenoptera. <Journ. N. Y. Ent. Soc., vol. 15, no. 4, p. 179, December.

1908. Some new Chalcidoidea. <Proc. Ent. Soc. Wash., vol. 9, pp. 157-160, April 6.

1909a. New Chalcidoidea. <Proc. Ent. Soc. Wash., vol. 11, p. 52.

1909b. Two new species of the genus Tetrastichus. <Proc. Ent. Soc. Wash., vol. 11 , p. 150 , October 5 .

$16844^{\circ}-$ Bull. $100-12-7$ 
Foster, F.

1908. The introduction of Iridomyrmex humilis (Mayr) into New Orleans. <Journ. Econ. Ent., vol. 1, no. 5, pp. 289-293, October 5.

Girault, $\Lambda$. Arsene.

1907. The lesser peach borer. <U. S. Dept. Agr., Bur. Ent., Bul. 68, Pt. IV, p. 45 .

Contains an undoubtedly erroneous reference to (Bracon) MI icrobracon melli/or Say.

Herrera, Armonso L.

$1902 a$. Sexto informe acerca del picudo del Algodon (Insanthonomus grandis 1. C. $\mathrm{Cu}_{\text {.) }}$ : Pequeña avispa parásita del picudo. <Boletín de la Comisión de Parasitología Agrícola, vol. 1, no. 9, pp.403-404. This is a translation of $\mathrm{Ash}$ shead $1902 \mathrm{~b}$.

1902b. Hormiga destructora del picudo. <Loc. cit., pp. 404-406. This contains a translation of Wheeler, 1902.

Hinds, WarRen Elmer.

1907 $\alpha$. An ant enemy of the cotton boll weevil. <U. S. Dept. Agr., Bur. Ent, Bul. 63, Pt. III, pp. 45-48, February 5.

1907b. Some factors in the natural control of the Mexican cotton boll weevil. $<$ U. S. Dept. Agr., Bur. Ent., Bul. 74, December 14.

Hood, Clarence Ellswortit.

1909. Types of cages found useful in parasite work. < Journ. Econ. Ent., vol. 2, no. 2, pp. 121-124, April 15.

Howard, Leland Ossian.

1897. The Mexican cotton boll weevil (Anthonomus grandis Boheman). <U. S Dept. Agr., Div. Ent., Cir. 18.

Hunter, Walter David.

1907. Some recent studies of the Mexican cotton boll weevil. <Yearbook U. S. Dept. Agr., 1906, pp. 318-322.

Hunter, Walter David, and Warren Elmer Hinds,

1904. The Mexican cotton boll weevil. <U. S. Dept. Agr., Div. Ent., Bul. 45, pp. 104-110.

List of parasites and predators; adds as new records Sigalphus curculionis Fitch, Catolaccus incertus A shmead, Urosigalphus (robustus Ashmead), Bracon (dorsator Say), and Eurytoma tylodermatis Ashmead, as well as an entomophagons fungus, Aspcrgillus sp.

1905. The Mexican cotton boll weevil. <U. S. Dept. Agr., Bur. Ent., Bul. 51, pp. 143-150.

Hunter, Walter David, Wilmon Newell, and William Dwight Piferce.

1907. The insect enemies of the boll weevil. <Louisiana Crop Pest Comm., Cir. 20, December.

Maliy, Frederick William.

1902. Report on the boll weevil. <Public Printer, Austin, Tex., pp. 23-24.

Records (Bracon) Microbracon mellitor Say, Ccrambycobius cyaniceps Ashmead, and Eurytoma sp. from the boll weevil.

Morgan, Alpred Cookman.

1907. A predatory bug reported as an enemy of the cotton boll weevil. <U. S. Dept. Agr., Bur. Ent., Bul. 63, Pt. IV, pp. 49-54, February 8.

Neweil, Wilmon, and R. C. Tremearne.

1908. A new predaccous enemy of the cotton boll weevil. < Journ. Econ. Ent., vol. 1, no. 4, p. 244, August 15. 


\section{Pierce, William Dwight.}

1907a. Notes on the biology of certain weevils related to the cotton boll weevil. <U. S. Dept. Agr., Bur. Ent., Bul. 63, Pt. II, pp. 37-44, February 5.

1907b. On the biologies of the Rhynchophora of North America. <Ann. Rept. Nebraska State Board Agr., 1906-7, pp. 261, 263, 268, 269, 270, 271, $272,274,276,278,279,282,283,285,295$, September.

1907c. Contributions to the knowledge of Rhynchophora. <Ent. News, vol. 18, pp. 356-363 (October), 379-385 (November).

1908a. Studies of parasites of the cotton boll weevil. <U. S. Dept. Agr., Bur. Ent., Bul. 73, p. 63, January 21.

$1908 b$. The economic bearing of recent studies of the parasites of the cotton boll weevil. <Journ. Econ. Ent., vol. 1, no. 2, pp. 117-122, April 15.

1908c. Factors controlling parasitism with special reference to the cotton boll weevil. <Jourm. Econ. Ent., vol, 1, no. 5, pp. 315-323, October 15.

1908d. A list of parasites known to attack American Rhynchophora. <Journ. Econ. Ent., vol. 1, no. 6, pp. 380-396, December 15.

1910. On some phases of parasitism displayed by insect enemies of weevils. $<$ Journ. Econ. Ent, vol. 3, pp. 451-488, December 15.

Pratt, Frederick Charles.

1907. Notes on the pepper weevil. <U. S. Dept. Agr., Bur. Ent., Bul.63, Pt. V, p. 56 , February 9 .

RANGEL, A. F.

1901a. Segundo informe acerca del picudo del algodon (Insanthonomus grandis I. C. Cu.). <Boletín de la Comisión de Parasitología Agrícola, vol. 1, no. 5, pp. 171-176.

1901b. Tercer informe acerca del picudo del algodon. <Boletín de la Comisión de Parasitología Agrícola, vol. 1, no. 6, pp. 197-206.

1901c. Cuarto informe acerca del picudo del algodon (Insanthonomus grandis I. C. Cu.). < Boletín de la Comisión de Parasitología Agrícola, vol. 1, no. 7, pp. 245-261.

Rilex, Cmarles Vaeentine, and Lbland Ossian Howard.

1890. Some of the bred parasitic Hymenoptera in the National Museum collection. <U. S. Dept. Agr., Div. Ent., Ins. Life, vol. 2, pp. 348-350, 353 ; vol. 4, pp. 122, 123.

Schivarz, Eugene Amandus.

1905. Note on Dorymyrmex pyramicus Roger. <Proc. Ent. Soc. Wash., vol. 7, p. 4. Townsend, Charles Henry Tyler.

1895. Reports on the Mexican cotton boll weevil in Texas (Anthonomus grandis Boh.). <U. S. Dept. Agr., Diy. Ent., Ins. Life, vol. 7, pp. 295-309.

1908. The taxonomy of the muscoidean flies, including descriptions of new genera and species. <Smithsonian Misc. Coll., vol. 51, no. 1803, pp. 56-58, 86.

Wheeler, William Morton.

1902. Formica fusea Linnæus, subsp. subpolita Miayr., var. perpilosa n. var. $<$ Boletín de la Comisión de Parasitología Agrícola, vol. 1, no. 9, pp. 406-407. (For Spanish translation see Herrera.) 







\section{LIBRARY OF CONGRESS

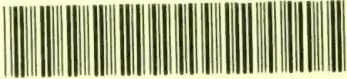

00008884316 